\title{
The colorectal response to butyrate in health and IBS
}

Citation for published version (APA):

Vanhoutvin, S. (2014). The colorectal response to butyrate in health and IBS. [Doctoral Thesis, Maastricht University]. Datawyse / Universitaire Pers Maastricht. https://doi.org/10.26481/dis.20141106sv

Document status and date:

Published: 01/01/2014

DOI:

10.26481/dis.20141106sv

Document Version:

Publisher's PDF, also known as Version of record

\section{Please check the document version of this publication:}

- A submitted manuscript is the version of the article upon submission and before peer-review. There can be important differences between the submitted version and the official published version of record.

People interested in the research are advised to contact the author for the final version of the publication, or visit the DOI to the publisher's website.

- The final author version and the galley proof are versions of the publication after peer review.

- The final published version features the final layout of the paper including the volume, issue and page numbers.

Link to publication

\footnotetext{
General rights rights.

- You may freely distribute the URL identifying the publication in the public portal. please follow below link for the End User Agreement:

www.umlib.nl/taverne-license

Take down policy

If you believe that this document breaches copyright please contact us at:

repository@maastrichtuniversity.nl

providing details and we will investigate your claim.
}

Copyright and moral rights for the publications made accessible in the public portal are retained by the authors and/or other copyright owners and it is a condition of accessing publications that users recognise and abide by the legal requirements associated with these

- Users may download and print one copy of any publication from the public portal for the purpose of private study or research.

- You may not further distribute the material or use it for any profit-making activity or commercial gain

If the publication is distributed under the terms of Article $25 \mathrm{fa}$ of the Dutch Copyright Act, indicated by the "Taverne" license above, 
The colorectal response to butyrate in health and Irritable Bowel Syndrome

Steven Vanhoutvin 

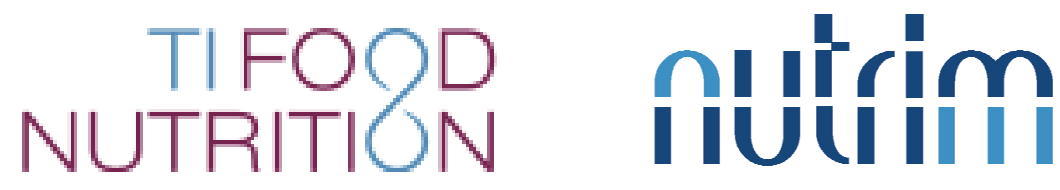

The studies presented in this thesis were performed within TI Food and Nutrition, and NUTRIM, School for Nutrition, Toxicology and Metabolism, accredited by the Royal Netherlands Academy of Arts and Sciences.

Copyright Steven Vanhoutvin, Maastricht 2014

Layout: Tiny Wouters

Coverdesign: Dana Hamers, Scientific Art

Graphics: Steven en Bart Vanhoutvin

Production: Datawyse | Universitaire Pers Maastricht

ISBN: 978-94-6159-374-0 


\title{
The colorectal response to butyrate in health and IBS
}

\section{PROEFSCHRIFT}

\author{
ter verkrijging van de graad van doctor aan de Universiteit Maastricht \\ op gezag van de Rector Magnificus, Prof. dr. L.L.G. Soete \\ volgens het besluit van het College van Decanen, \\ in het openbaar te verdedigen \\ op donderdag 6 november 2014 om 14.00 uur
}

door

Steven Vanhoutvin

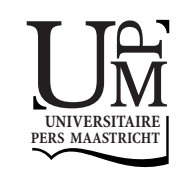




\section{Promotor:}

Prof. dr. A.A.M. Masclee

Prof. dr. R-J.M. Brummer, Dean Faculty of Medicine and Health, Örebro University, Sweden

\section{Co-promotores:}

Dr. F. Troost

Dr. D.M.A.E. Jonkers

\section{Beoordelingscommissie:}

Prof. dr. W. Saris, voorzitter

Prof. dr. C. Baeten

Dr. R. Felt Bersma, VU Medisch Centrum, Amsterdam

Prof. dr. F. Brouns

Prof. dr. F.J. van Schooten 


\section{Contents}

Chapter $1 \quad$ General introduction

Chapter 2 Explorative studies in humans on techniques with targeted delivery of substrates to the distal gut

Chapter 3 Butyrate-induced transcriptional changes in human colonic mucosa

PLoS One. 2009;4:e6759

Chapter 4 The effects of butyrate on visceral perception in healthy volunteers

Neurogastroenterol Motil. 2009;21:952-e76

Chapter $5 \quad$ Rectal butyrate instillation decreases visceral perception in IBS patients

Submitted

Chapter 6 Alternative procedure to shorten rectal barostat procedure for the assessment of rectal compliance and visceral perception: a feasibility study

J Gastroenterol 2012;47:896-903

Chapter $7 \quad$ Summary and general discussion

Samenvatting

Valorisation

Dankwoord

Curriculum vitae + publicatielijst 
1

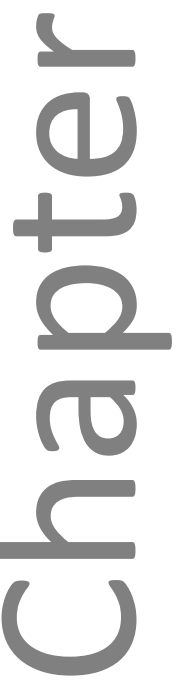


General introduction 


\section{Introduction}

\section{The human colon}

The colon represents the most distal part of the gastrointestinal tract and connects the distal ileum and cecal valve with the rectum. Main functions of the colon include storage and concentration of luminal content by absorption of water and electrolytes. Furthermore, the colon provides an anaerobic environment, which harbours a complex microbial ecosystem that contributes to maintaining gut homeostasis of the host. For example, microbial fermentation of non-digestible carbohydrates provides substrates that can readily be metabolized by the colonic mucosa.

\section{Colonic microbiota and ecosystem}

The human body harbours ten times more microbial cells than the total number of human cells. The oral cavity to the colon contains a proximal to distal gradient over which the number of bacteria per $\mathrm{ml}$ substantially increases. The majority of bacteria is found in the human colon, with a density of more than $10^{10}-10^{12}$ bacteria per gram luminal content. Over 1000 different microbial species have already been identified in the colon $^{1,2}$. The intestinal microbiome has several important functions such as colonisation resistance, fermentation of otherwise non-digestible macronutrients resulting in production of short chain fatty acids (SCFAs), production of vitamins and modulation of the host epithelial barrier and immune system. Most micro-organisms preferentially ferment carbohydrates. However, the gut microbiota is able to switch to protein fermentation in case of depletion of carbohydrates availability. Changes in composition of intestinal microbiota and specific microbial profiles have been associated with specific gastrointestinal diseases. An aberrant immune response towards commensal bacteria has been suggested to be a causal factor in the development of inflammatory bowel disease (IBD). In these patients, both a difference in the composition and in the metabolic activity of the intestinal microbiota have been reported $^{3}$. In irritable bowel sydrome (IBS), altered composition of the microbiota, that may result from a previous infection or from treatment with antibiotics, has also been hypothesised to play a role in the etiology and pathogenesis of the disorder ${ }^{4-6}$.

\section{Prebiotics}

Both the microbiota and several end products of carbohydrate fermentation are thought to exert health promoting effects. As stated above, fermentation of nondigestible carbohydrates by colonic microbiota results in the production of metabolites such as SCFAs. Much effort has been put into developing strategies to influence and modify both the microbial composition and the production of SCFAs, as they may exert health promoting effects. Several definitions for a prebiotic compound are currently used such as: "a selectively fermented ingredient that allows specific 
changes, both in the composition and/or activity in the gastrointestinal microbiota, that confers health benefits" but also "a non-digestible food ingredient that beneficially affects host health by selectively stimulating the growth and/or activity of one or a limited number of bacteria in the colon" ${ }^{7}$. Non-digestible carbohydrates are resistant to degaradation by gastric acid and by enzymes in the small intestine and thus reach the colon intact, where they are fermented by colonic microbiota.

The beneficial effects resulting from prebiotic treatments include an increase in bifido bacteria, improvement of stool habits ( $\mathrm{pH}$, stool frequency and consistency), and an increase of SCFA concentrations. Other potentially beneficial effects of prebiotics include a reduction in the risk of gastroenteritis, IBS, diabetes and obesity, and can have a preventive effect on colon cancer ${ }^{7}$.

Apart from the amount and type of dietary fiber (chain length, solubility and branches), the microbiome in the colon also affects the speed of fermentation and thereby the local production of SCFAs. The amount of daily recommended fiber intake ranges between 30-40g. In the Netherlands, our Western type diet contains an average of $21 \mathrm{~g}$ of dietary fibers ${ }^{8,9}$ and is not considered sufficient for the production of adequate amounts of SCFAs throughout the entire colon. The lower fiber intake results in limited colonic carbohydrate availability, especially in the distal colon.

\section{SCFA: butyrate}

SCFAs derived from the microbial fermentation of undigested dietary fibers in the colon are mainly acetate, propionate and butyrate. The amounts that are produced depends on the substrate, site of fermentation, the diet and the composition of the microbiota, and can account for up to $5-15 \%$ of the total energy requirements in humans ${ }^{10}$. Fecal concentrations of acetate, propionate and butyrate are found in a molar ratio of approximately $60: 20: 20^{11,12}$, but data on luminal concentrations in specific parts of the colon are only available from a limited amount of observations in "sudden death" patients. Luminal concentrations of short chain fatty acids reported in literature vary from $137-197 \mathrm{mmol} / \mathrm{kg}$ chyme in the caecum to 86 to $97 \mathrm{mmol} / \mathrm{kg}$ chyme in the descending colon ${ }^{13,14}$. Due to rapid absorption and metabolism of intraluminal SCFAs, these data are probably not accurately representing the actual SCFA concentrations present in vivo in humans.

As micro-organisms preferably ferment carbohydrates, most saccharolytic fermentation occurs in the proximal colon. Depletion of carbohydrate sources in the distal colon leads to a switch from saccharolytic to proteolytic fermentation, which is less favorable due to the formation of potentially toxic products, such as ammonia, amines, phenols and sulphur containing compounds. These toxic products and the lower availability of SCFAs in the distal colon have been suggested as factors involved in the pathogenesis of gastrointestinal disorders such as ulcerative colitis (UC), IBS ${ }^{15,16}$ and cancer $^{17-20}$. Among the different SCFAs, butyrate is known to modulate numerous processes in the human body. It induces cell differentiation and strongly inhibits cell 
proliferation in tumor cell lines ${ }^{21-29}$. Colonocytes use butyrate as their primary energy source and in the absence of butyrate, colonocytes are prone to undergo apoptosis. Paradoxically, opposite effects have been observed in cancer cell lines where butyrate inhibits proliferation and increases apoptosis, suggesting a possible anti-carcinogenic effect of butyrate. The contrasting effects of butyrate on proliferation found in normal colonocytes and cancer cell lines are often referred to as the butyrate paradox, although this could mainly be explained by differences in concentration, medium, exposure time and cell cycle stage ${ }^{27,30-32}$. Furthermore, butyrate has a beneficial effect on inflammation ${ }^{27,29}$, oxidative stress ${ }^{27}$, intestinal barrier function ${ }^{27,33,34}$, and may play a role in satiety ${ }^{29,35,36}$. The exact mechanism by which butyrate exerts its effects are complex and are not yet fully elucidated. The most frequently described mechanism by which butyrate induces changes in gene transcription is by its ability to inhibit histon deacetylase. The inhibition of deacetylase leads to hyperacetylation of histone tails of nucleosomes, causing them to form a more open structure. This open structure subsequently facilitates the transcription of genes due to the increased accessibility of nucleosomal DNA to transcription factors.

Although a number of studies on transcriptional responses of butyrate have been studied in cell lines ${ }^{30,31,37-48}$, some studies have been performed in animals and to a limited extend also in IBD patients and healthy volunteers ${ }^{19,46,49-51}$. In vitro and animal studies indicate that butyrate downregulates the expression of genes associated with proliferation and oxidative stress and upregulates the expression of mucin associated genes (Muc 1-4), tight junction proteins (zonulin and occludin) and the butyrate transporter monocarboxylate transporter-1 (MCT1). In patients with UC, butyrate was shown to increase the expression of the butyrate transporter MCT-1 and to decrease inflammation by inhibition of the activation of NF- $\kappa$ B. Data on the transcriptional response in humans after butyrate intervention are limited. More research on transcriptional responses of butyrate treatment in humans may result in the identification of new leads for possible beneficial effects of butyrate and possible mechanisms of action for butyrate.

\section{Visceroperception in IBS}

Irritable bowel syndrome (IBS) is a heterogeneous and multifactorial disorder with a high prevalence among the general population. Up to $20 \%$ of subjects may suffer from abdominal symptoms and altered bowel habits ${ }^{52-54}$. Several factors are involved in the pathophysiology of IBS. These factors may act somewhere along the brain-gut-axis. With respect to the gut level, increased permeability, chronic low grade inflammation, increased numbers of (degranulating) mast cells, altered serotonin metabolism, gut microbial profile and visceral hypersensitivity have been reported in patients with $\mathrm{IBS}^{55-66}$. With respect to symptom generation such as abdominal pain, the intestinal mucosa is responsive to mechanical distension but not to other types of somatic stimuli, such as pain or heat. The response of the gut to this mechanical distension can 
be measured and quantified by barostat methodology. Upon introduction of a balloon into the rectum, further stepwise distension of the balloon, either pressure- or volume triggered, generates symptoms of urge, discomfort and pain. Subjects undergoing a barostat, score their symptoms on a Visual Analog Scale (VAS) at each distension step. In this way one is able to assess 1 ) thresholds for perception (reduced threshold for pain is referred to as allodynia) and 2) increased, higher scores for symptoms during the stepwise pressure- or volume distensions (hypersensitivity). An increased sensitivity to rectal balloon distension has been demonstrated in up to $60 \%$ of IBS patients ${ }^{67-69}$ and is considered an important hallmark of the disorder. The association between intestinal permeability, intestinal microbiota, metabolic processes in the lumen and visceral hypersensitivity in IBS needs further evaluation. Human studies on the effect of short chain fatty acids on visceral perception have not yet been published. To enable this, it is pivotal to reach consensus on the design of the barostat protocol, thus providing proper standardisation of the barostat technology to assess visceral sensitivity.

\section{Intestinal barrier}

One of the potential effects of SCFAs is its effect on barrier function. The association between a compromised intestinal barrier function and the development of a variety of diseases has recently received much attention. Diabetes ${ }^{70,71}$, food allergy ${ }^{72,73}$, celiac disease ${ }^{74,75}, \mathrm{IBD}^{76-79}$ and $\mathrm{IBS}^{55,56,58,60,65,79-83}$ have been associated with an increased intestinal permeability. The intestinal barrier function can be evaluated both at the molecular and at the functional level, each with its advantages and limitations. The most direct method to evaluate intestinal barrier function is to assess intestinal permeability by determining plasma- or urinary recovery of a combination of orally ingested permeation markers. This provides a well accepted and validated methodology to estimate intestinal barrier function ${ }^{72,84,85}$. Another method for studying the intestinal barrier function in vivo is to determine the expression and (co)localisation of tight junction proteins in intestinal mucosal tissue samples. Tight juctions are formed by a complex of different transmembrane- and intracellular proteins. The transmembrane proteins occludin and different claudins interact with the zonula occludens (ZO) proteins, which bind to the actin cytoskeleton. In concert with gap junctions (e.g. connexin), tight junction proteins form a network that connects to the intracellular cytoskeleton to modulate paracellular transit of molecules. Recent studies indicate that butyrate is able to modulate intestinal permeability by affecting the dynamic properties of tight junctions ${ }^{86,87}$.

Effects of a dietary intervention on intestinal barrier function can also be investigated using model systems such as confluent epithelial cell lines or Ussing chamber technology, in which freshly obtained mucosal tissue can be studied. Intestinal barrier function can then be determined using transepithelial flux of permeation markers, such as horseradish peroxidase (HRP), (51)CrEDTA or sucralose ${ }^{88}$, in combination with 
registration of changes in electrical conductance properties of the tissue ${ }^{88-90}$. The transepithelial resistance provides an estimation of the integrity of the intestinal tissue, in which a high resistance indicates a well maintained integrity of the tissue and a low resistance indicates a disturbed intestinal barrier function ${ }^{89}$.

\section{Aims and outline of investigations}

Studying human colon metabolism and function in vivo is challenging due to the limited accessibility of the colon. Studying the effects of drugs, food derived compounds, bacteria, and bacterial metabolites in vivo in humans has been hampered by the available tools for colon-specific delivery. Such tools should preferably not require prior bowel cleansing, since this affects the microbial community, induces fluid fluxes and most likely induces local stress to the mucosa and hence may affect colonic function and mucosal gene expression. Furthermore, a suitable tool for targeted delivery should include the option to carry a sufficient amount of substrate or drugs and should reliably release its contents in the targeted area. In chapter 2 , the targeted delivery of substrates to a) the proximal colon by enteric-coated capsules, b) to the distal ileum by an oro-ileal catheter and c) to the distal colon by rectal enemas was tested in three separate explorative studies in healthy volunteers.

The short chain fatty acid butyrate is known to inhibit histone deacetylase and several animal and in vitro data show that it can affect numerous processes via this mechanism of action. More research is needed to study the effects of butyrate on gene transcription in vivo in humans, to obtain overview of the mechanisms of action of butyrate and processes involved, possibly leading to its proven health benefits. In chapter 3 the effects of rectally administered butyrate at a concentration of $100 \mathrm{mM}$, reflecting colonic concentrations in the physiological range, on gene transcription were studied in healthy volunteers using genome-wide microarray analyses.

Previously, positive effects of butyrate on inflammation and on gastrointestinal symptoms in patients with active distal ulcerative colitis have been reported ${ }^{91,92}$. Two studies in rats however, have pointed to an increased visceral sensitivity after rectal butyrate instillation ${ }^{93,94}$. It is not known whether butyrate administration in healthy humans would have a potentially beneficial effect on visceral sensitivity (via yet unknown mechanisms) or would increase visceral sensitivity as shown in the rat studies. Chapter 4 describes a study on the effects of rectally administered butyrate, in two concentrations (50 $\mathrm{mM}$ and $100 \mathrm{mM}$ ) on visceral sensitivity in healthy volunteers. Visceral perception was determined using a barostat procedure ${ }^{95}$.

Increased visceral sensitivity is an important hallmark of- and a potential factor in the pathophysiology of IBS. An increased sensitivity to rectal balloon distension has been 
demonstrated in up to $60 \%$ of IBS patients in several studies ${ }^{68,69,96,97}$. In addition, a reduction in rectal compliance was found in IBS patients versus controls ${ }^{98,99}$. The increased visceral perception in IBS patients is associated with daily symptoms (e.g. pain and urge to defecate), reduced quality of life, anxiety disorder and depression ${ }^{69,100}$. Local application or oral ingestion of dietary ingredients that modify visceral perception and thereby reduce daily symptoms and complaints in IBS patients may provide an attractive alternative or additional therapeutic strategy in IBS patients. Based on the visceroperception data obtained in healthy volunteers we performed a study on the effect of rectal butyrate instillation on visceral sensitvity in IBS patients (chapter 5) and compared the effects found in IBS patients with those observed in healthy volunteers.

We anticipate that changes in visceroperception are related to local changes in gut barrier function and permeability. A potential beneficial effect of butyrate on visceroperception may result from an improved barrier function. Therefore, in that chapter we also investigate in the ex vivo model with Ussing chambers the preventive effect of butyrate on a stressor-induced increase in intestinal permeability.

Visceral perception is generally measured in vivo using the barostat technique. Since the introduction of the barostat methodology, different distension protocols have been used and efforts have been made to optimise and standardise the distension protocols $^{101-103}$. However, currently there is no general consensus on the most optimal protocol. We propose a shortened barostat procedure in an attempt to minimise patient burden while at the same time reducing the risk that human errors may occur during the measurement procedure. This procedure is described in chapter 6 .

In the concluding chapter 7 , the various findings of the studies described in this thesis are summarised and discussed to be put in the perspective of the overall effects of butyrate on gut health. 


\section{References}

1. Zoetendal EG, Rajilic-Stojanovic M, de Vos WM. High-throughput diversity and functionality analysis of the gastrointestinal tract microbiota. Gut 2008;57:1605-15.

2. Sekirov I, Russell SL, Antunes LC, et al. Gut microbiota in health and disease. Physiological reviews;90:859-904.

3. Fava F, Danese S. Intestinal microbiota in inflammatory bowel disease: friend of foe? World J Gastroenterol 2011;17:557-66.

4. Crouzet L, Gaultier E, Del'Homme C, et al. The hypersensitivity to colonic distension of IBS patients can be transferred to rats through their fecal microbiota. Neurogastroenterol Motil;25:e272-82.

5. Ohman L, Simren M. Intestinal microbiota and its role in irritable bowel syndrome (IBS). Current gastroenterology reports;15:323.

6. Carroll IM, Ringel-Kulka T, Siddle JP, et al. Alterations in composition and diversity of the intestinal microbiota in patients with diarrhea-predominant irritable bowel syndrome. Neurogastroenterol Motil 2012;24:521-30, e248.

7. Slavin J. Fiber and prebiotics: mechanisms and health benefits. Nutrients 2013;5:1417-35.

8. Rossum CTM van FH, Verkaik-Kloosterman J, Buurma-Rethans EJM, Ocke MC. Dutch National Food consumption survey 2007-2010: Diet of children and adults aged 7 to 69 years. RIVM Rapportnr 350050006, Bilthoven. 2011.

9. Voedingscentrum. Zo eet Nederland. resultaten van voedselconsumptiepeiling 1997-1998 Den Haag. 1998.

10. Bergman EN. Energy contributions of volatile fatty acids from the gastrointestinal tract in various species. Physiological reviews 1990;70:567-90.

11. Topping DL, Clifton PM. Short-chain fatty acids and human colonic function: roles of resistant starch and nonstarch polysaccharides. Physiological reviews 2001;81:1031-64.

12. Hallert C, Bjorck I, Nyman M, et al. Increasing fecal butyrate in ulcerative colitis patients by diet: controlled pilot study. Inflammatory bowel diseases 2003;9:116-21.

13. Cummings JH, Pomare EW, Branch WJ, et al. Short chain fatty acids in human large intestine, portal, hepatic and venous blood. Gut 1987;28:1221-7.

14. Macfarlane GT, Gibson GR, Cummings JH. Comparison of fermentation reactions in different regions of the human colon. The Journal of applied bacteriology 1992;72:57-64.

15. Kannampalli $P$, Shaker R, Sengupta JN. Colonic butyrate- algesic or analgesic? Neurogastroenterol Motil 2011;23:975-9.

16. Ghaith O, El-Halabi M, Hashash JG, et al. Investigational agents for the irritable bowel syndrome. Expert opinion on investigational drugs;19:1161-78.

17. Marteau P. Probiotics, prebiotics, synbiotics: ecological treatment for inflammatory bowel disease? Gut 2006;55:1692-3.

18. Le Leu RK, Brown IL, Hu Y, et al. Effect of dietary resistant starch and protein on colonic fermentation and intestinal tumourigenesis in rats. Carcinogenesis 2007;28:240-5.

19. Wong CS, Sengupta S, Tjandra JJ, et al. The influence of specific luminal factors on the colonic epithelium: high-dose butyrate and physical changes suppress early carcinogenic events in rats. Dis Colon Rectum 2005;48:549-59.

20. Vigsnaes LK, van den Abbeele P, Sulek K, et al. Microbiotas from UC patients display altered metabolism and reduced ability of LAB to colonize mucus. Scientific reports;3:1110.

21. Comalada $M$, Bailon $E$, de Haro $O$, et al. The effects of short-chain fatty acids on colon epithelial proliferation and survival depend on the cellular phenotype. Journal of cancer research and clinical oncology 2006;132:487-97.

22. Ranganna K, Joshi T, Yatsu FM. Sodium butyrate inhibits platelet-derived growth factor-induced proliferation of vascular smooth muscle cells. Arteriosclerosis, thrombosis, and vascular biology 1995;15:2273-83.

23. Ranganna K, Yatsu FM, Hayes BE, et al. Butyrate inhibits proliferation-induced proliferating cell nuclear antigen expression (PCNA) in rat vascular smooth muscle cells. Molecular and cellular biochemistry 2000;205:149-61. 
24. Prasad KN. Butyric acid: a small fatty acid with diverse biological functions. Life sciences 1980;27:1351-8.

25. Hague A, Butt AJ, Paraskeva C. The role of butyrate in human colonic epithelial cells: an energy source or inducer of differentiation and apoptosis? Proc Nutr Soc 1996;55:937-43.

26. Hodin RA, Meng S, Archer S, et al. Cellular growth state differentially regulates enterocyte gene expression in butyrate-treated HT-29 cells. Cell Growth Differ 1996;7:647-53.

27. Hamer HM, Jonkers D, Venema K, et al. Review article: the role of butyrate on colonic function. Aliment Pharmacol Ther 2008;27:104-19.

28. Zhang S, Bai J, Ren S, et al. Sodium butyrate restores ASC expression and induces apoptosis in LS174T cells. International journal of molecular medicine;30:1431-7.

29. Canani RB, Costanzo MD, Leone L, et al. Potential beneficial effects of butyrate in intestinal and extraintestinal diseases. World J Gastroenterol 2011;17:1519-28.

30. Cuff $\mathrm{M}$, Dyer J, Jones $\mathrm{M}$, et al. The human colonic monocarboxylate transporter Isoform 1: its potential importance to colonic tissue homeostasis. Gastroenterology 2005;128:676-86.

31. Pajak B, Orzechowski A, Gajkowska B. Molecular basis of sodium butyrate-dependent proapoptotic activity in cancer cells. Advances in medical sciences 2007;52:83-8.

32. Donohoe DR, Collins LB, Wali A, et al. The Warburg effect dictates the mechanism of butyratemediated histone acetylation and cell proliferation. Molecular cell;48:612-26.

33. Mariadason JM, Kilias D, Catto-Smith A, et al. Effect of butyrate on paracellular permeability in rat distal colonic mucosa ex vivo. J Gastroenterol Hepatol 1999;14:873-9.

34. Peng L, He Z, Chen W, et al. Effects of butyrate on intestinal barrier function in a Caco-2 cell monolayer model of intestinal barrier. Pediatric research 2007;61:37-41.

35. Cherbut C. Motor effects of short-chain fatty acids and lactate in the gastrointestinal tract. Proc Nutr Soc 2003;62:95-9.

36. Cani PD, Joly E, Horsmans $\mathrm{Y}$, et al. Oligofructose promotes satiety in healthy human: a pilot study. European journal of clinical nutrition 2006;60:567-72.

37. Daly K, Shirazi-Beechey SP. Microarray analysis of butyrate regulated genes in colonic epithelial cells. DNA Cell Biol 2006;25:49-62.

38. Li RW, Li C. Butyrate induces profound changes in gene expression related to multiple signal pathways in bovine kidney epithelial cells. BMC Genomics 2006;7:234.

39. Daly K, Cuff MA, Fung F, et al. The importance of colonic butyrate transport to the regulation of genes associated with colonic tissue homoeostasis. Biochem Soc Trans 2005;33:733-5.

40. Li CJ, Li RW, Wang YH, et al. Pathway analysis identifies perturbation of genetic networks induced by butyrate in a bovine kidney epithelial cell line. Functional \& integrative genomics 2007;7:193-205.

41. Ranganna K, Yousefipour Z, Yatsu FM, et al. Gene expression profile of butyrate-inhibited vascular smooth muscle cell proliferation. Molecular and cellular biochemistry 2003;254:21-36.

42. Tabuchi Y, Takasaki I, Doi T, et al. Genetic networks responsive to sodium butyrate in colonic epithelial cells. FEBS Lett 2006;580:3035-41.

43. Joseph J, Mudduluru G, Antony S, et al. Expression profiling of sodium butyrate (NaB)-treated cells: identification of regulation of genes related to cytokine signaling and cancer metastasis by $\mathrm{NaB}$. Oncogene 2004;23:6304-15.

44. Mariadason JM, Corner GA, Augenlicht LH. Genetic reprogramming in pathways of colonic cell maturation induced by short chain fatty acids: comparison with trichostatin A, sulindac, and curcumin and implications for chemoprevention of colon cancer. Cancer Res 2000;60:4561-72.

45. Miller SJ, Zaloga GP, Hoggatt AM, et al. Short-chain fatty acids modulate gene expression for vascular endothelial cell adhesion molecules. Nutrition 2005;21:740-8.

46. Thibault R, De Coppet $\mathrm{P}$, Daly $\mathrm{K}$, et al. Down-regulation of the monocarboxylate transporter 1 is involved in butyrate deficiency during intestinal inflammation. Gastroenterology 2007;133:1916-27.

47. Williams EA, Coxhead JM, Mathers JC. Anti-cancer effects of butyrate: use of micro-array technology to investigate mechanisms. Proc Nutr Soc 2003;62:107-15.

48. Bordin M, D'Atri F, Guillemot L, et al. Histone deacetylase inhibitors up-regulate the expression of tight junction proteins. Mol Cancer Res 2004;2:692-701.

49. Kien CL, Blauwiekel R, Bunn JY, et al. Cecal infusion of butyrate increases intestinal cell proliferation in piglets. J Nutr 2007;137:916-22. 
50. Kien CL, Peltier CP, Mandal S, et al. Effects of the in vivo supply of butyrate on histone acetylation of cecum in piglets. Jpen 2008;32:51-6.

51. Gaudier E, Rival M, Buisine $\mathrm{M}$, et al. Butyrate enemas upregulate Muc genes expression but decrease adherent mucus thickness in mice colon. Physiol Res 2008.

52. Boekema PJ, van Dam van Isselt EF, Bots ML, et al. Functional bowel symptoms in a general Dutch population and associations with common stimulants. The Netherlands journal of medicine 2001;59:23-30

53. Chang L. Review article: epidemiology and quality of life in functional gastrointestinal disorders. Aliment Pharmacol Ther 2004;20 Suppl 7:31-9.

54. Chang L, Heitkemper MM. Gender differences in irritable bowel syndrome. Gastroenterology 2002;123:1686-701.

55. Barbara G, Zecchi L, Barbaro R, et al. Mucosal permeability and immune activation as potential therapeutic targets of probiotics in irritable bowel syndrome. J Clin Gastroenterol 2012;46 Suppl:S525.

56. Wilcz-Villega EM, McClean S, O'Sullivan MA. Mast Cell Tryptase Reduces Junctional Adhesion Molecule-A (JAM-A) Expression in Intestinal Epithelial Cells: Implications for the Mechanisms of Barrier Dysfunction in Irritable Bowel Syndrome. Am J Gastroenterol 2013;108:1140-51

57. Bertiaux-Vandaele N, Youmba SB, Belmonte L, et al. The expression and the cellular distribution of the tight junction proteins are altered in irritable bowel syndrome patients with differences according to the disease subtype. Am J Gastroenterol;106:2165-73.

58. Camilleri M, Gorman H. Intestinal permeability and irritable bowel syndrome. Neurogastroenterol Motil 2007; 19:545-52.

59. Camilleri M, Lasch K, Zhou W. Irritable bowel syndrome: methods, mechanisms, and pathophysiology. The confluence of increased permeability, inflammation, and pain in irritable bowel syndrome. $\mathrm{Am} J$ Physiol Gastrointest Liver Physiol 2012;303:G775-85.

60. Lee H, Park JH, Park DI, et al. Mucosal mast cell count is associated with intestinal permeability in patients with diarrhea predominant irritable bowel syndrome. Journal of neurogastroenterology and motility 2013;19:244-50.

61. Zhou Q, Zhang B, Verne GN. Intestinal membrane permeability and hypersensitivity in the irritable bowel syndrome. Pain 2009;146:41-6.

62. Camilleri M. Serotonin in the gastrointestinal tract. Current opinion in endocrinology, diabetes, and obesity 2009;16:53-9.

63. Kilkens TO, Honig A, van Nieuwenhoven MA, et al. Acute tryptophan depletion affects brain-gut responses in irritable bowel syndrome patients and controls. Gut 2004;53:1794-800.

64. Spiller R. Serotonin and GI clinical disorders. Neuropharmacology 2008;55:1072-80.

65. Lee JW, Park JH, Park DI, et al. Subjects with diarrhea-predominant IBS have increased rectal permeability responsive to tryptase. Dig Dis Sci 2010;55:2922-8.

66. Park JH, Rhee PL, Kim HS, et al. Mucosal mast cell counts correlate with visceral hypersensitivity in patients with diarrhea predominant irritable bowel syndrome. J Gastroenterol Hepatol 2006;21:71-8.

67. Ludidi S, Conchillo JM, Keszthelyi D, et al. Does meal ingestion enhance sensitivity of visceroperception assessment in irritable bowel syndrome? Neurogastroenterol Motil 2012;24:47-53, e3.

68. Ludidi S, Conchillo JM, Keszthelyi D, et al. Rectal hypersensitivity as hallmark for irritable bowel syndrome: defining the optimal cutoff. Neurogastroenterol Motil 2012;24:729-33, e345-6.

69. van der Veek PP, Van Rood YR, Masclee AA. Symptom severity but not psychopathology predicts visceral hypersensitivity in irritable bowel syndrome. Clin Gastroenterol Hepatol 2008;6:321-8.

70. Sapone A, de Magistris L, Pietzak M, et al. Zonulin upregulation is associated with increased gut permeability in subjects with type 1 diabetes and their relatives. Diabetes 2006;55:1443-9.

71. Watts T, Berti I, Sapone A, et al. Role of the intestinal tight junction modulator zonulin in the pathogenesis of type I diabetes in BB diabetic-prone rats. Proceedings of the National Academy of Sciences of the United States of America 2005;102:2916-21.

72. Andre C, Andre F, Colin L, et al. Measurement of intestinal permeability to mannitol and lactulose as a means of diagnosing food allergy and evaluating therapeutic effectiveness of disodium cromoglycate. Annals of allergy 1987;59:127-30. 
73. Forbes EE, Groschwitz K, Abonia JP, et al. IL-9- and mast cell-mediated intestinal permeability predisposes to oral antigen hypersensitivity. J Exp Med 2008;205:897-913.

74. Drago S, El Asmar R, Di Pierro M, et al. Gliadin, zonulin and gut permeability: Effects on celiac and non-celiac intestinal mucosa and intestinal cell lines. Scand J Gastroenterol 2006;41:408-19.

75. Fasano A, Not T, Wang W, et al. Zonulin, a newly discovered modulator of intestinal permeability, and its expression in coeliac disease. Lancet 2000;355:1518-9.

76. Gibson PR. Increased gut permeability in Crohn's disease: is TNF the link? Gut 2004;53:1724-5.

77. Su L, Shen L, Clayburgh DR, et al. Targeted epithelial tight junction dysfunction causes immune activation and contributes to development of experimental colitis. Gastroenterology 2009;136:55163.

78. Suenaert $\mathrm{P}$, Bulteel V, Lemmens $\mathrm{L}$, et al. Anti-tumor necrosis factor treatment restores the gut barrier in Crohn's disease. Am J Gastroenterol 2002;97:2000-4.

79. Vivinus-Nebot $M$, Frin-Mathy $G$, Bzioueche $H$, et al. Functional bowel symptoms in quiescent inflammatory bowel diseases: role of epithelial barrier disruption and low-grade inflammation. Gut 2013.

80. Dunlop SP, Hebden J, Campbell E, et al. Abnormal intestinal permeability in subgroups of diarrheapredominant irritable bowel syndromes. Am J Gastroenterol 2006;101:1288-94.

81. Gecse K, Roka R, Ferrier L, et al. Increased faecal serine protease activity in diarrhoeic IBS patients: a colonic lumenal factor impairing colonic permeability and sensitivity. Gut 2008;57:591-9.

82. Piche T, Barbara G, Aubert P, et al. Impaired intestinal barrier integrity in the colon of irritable bowel syndrome patients: involvement of soluble mediators. Gut 2008.

83. Turcotte JF, Kao D, Mah SJ, et al. Breaks in the wall: increased gaps in the intestinal epithelium of irritable bowel syndrome patients identified by confocal laser endomicroscopy (with videos). Gastrointest Endosc 2013;77:624-30.

84. Lennernas H. Human intestinal permeability. J Pharm Sci 1998;87:403-10.

85. Anderson $A D$, Jain PK, Fleming $S$, et al. Evaluation of a triple sugar test of colonic permeability in humans. Acta Physiol Scand 2004;182:171-7.

86. Ohata A, Usami M, Miyoshi M. Short-chain fatty acids alter tight junction permeability in intestinal monolayer cells via lipoxygenase activation. Nutrition 2005;21:838-47.

87. Peng L, Li ZR, Green RS, et al. Butyrate Enhances the Intestinal Barrier by Facilitating Tight Junction Assembly via Activation of AMP-Activated Protein Kinase in Caco-2 Cell Monolayers. J Nutr 2009.

88. Wallon $C$, Braaf $Y$, Wolving $M$, et al. Endoscopic biopsies in Ussing chambers evaluated for studies of macromolecular permeability in the human colon. Scand J Gastroenterol 2005;40:586-95.

89. Li H, Sheppard DN, Hug MJ. Transepithelial electrical measurements with the Ussing chamber. J Cyst Fibros 2004;3 Suppl 2:123-6.

90. Song JP, Chen X, Yang G, et al. Corticotropin releasing hormone activates CD14 cells to induce endothelial barrier dysfunction. Cell biology international 2013.

91. Scheppach W, Sommer H, Kirchner T, et al. Effect of butyrate enemas on the colonic mucosa in distal ulcerative colitis. Gastroenterology 1992;103:51-6.

92. Vernia P, Marcheggiano A, Caprilli R, et al. Short-chain fatty acid topical treatment in distal ulcerative colitis. Aliment Pharmacol Ther 1995;9:309-13.

93. Bourdu S, Dapoigny M, Chapuy E, et al. Rectal instillation of butyrate provides a novel clinically relevant model of noninflammatory colonic hypersensitivity in rats. Gastroenterology 2005;128:19962008.

94. Tarrerias AL, Millecamps M, Alloui A, et al. Short-chain fatty acid enemas fail to decrease colonic hypersensitivity and inflammation in TNBS-induced colonic inflammation in rats. Pain 2002;100:91-7.

95. Whitehead WE, Delvaux M. Standardization of barostat procedures for testing smooth muscle tone and sensory thresholds in the gastrointestinal tract. The Working Team of Glaxo-Wellcome Research, UK. Dig Dis Sci 1997;42:223-41.

96. Mertz H, Naliboff B, Munakata J, et al. Altered rectal perception is a biological marker of patients with irritable bowel syndrome. Gastroenterology 1995;109:40-52.

97. Barbara G, Cremon C, De Giorgio R, et al. Mechanisms underlying visceral hypersensitivity in irritable bowel syndrome. Current gastroenterology reports;13:308-15.

98. Azpiroz F, Bouin M, Camilleri M, et al. Mechanisms of hypersensitivity in IBS and functional disorders. Neurogastroenterol Motil 2007;19:62-88. 
99. Kanazawa M, Palsson OS, Thiwan SI, et al. Contributions of pain sensitivity and colonic motility to IBS symptom severity and predominant bowel habits. Am J Gastroenterol 2008;103:2550-61.

100. Drossman DA, Camilleri M, Mayer EA, et al. AGA technical review on irritable bowel syndrome. Gastroenterology 2002;123:2108-31.

101. Krogh K, Ryhammer AM, Lundby L, et al. Comparison of methods used for measurement of rectal compliance. Dis Colon Rectum 2001;44:199-206.

102. Fox M, Thumshirn M, Fried $M$, et al. Barostat measurement of rectal compliance and capacity. Dis Colon Rectum 2006;49:360-70.

103. Park JH, Baek YH, Park DI, et al. Analysis of rectal dynamic and static compliances in patients with irritable bowel syndrome. Int J Colorectal Dis 2008;23:659-64. 

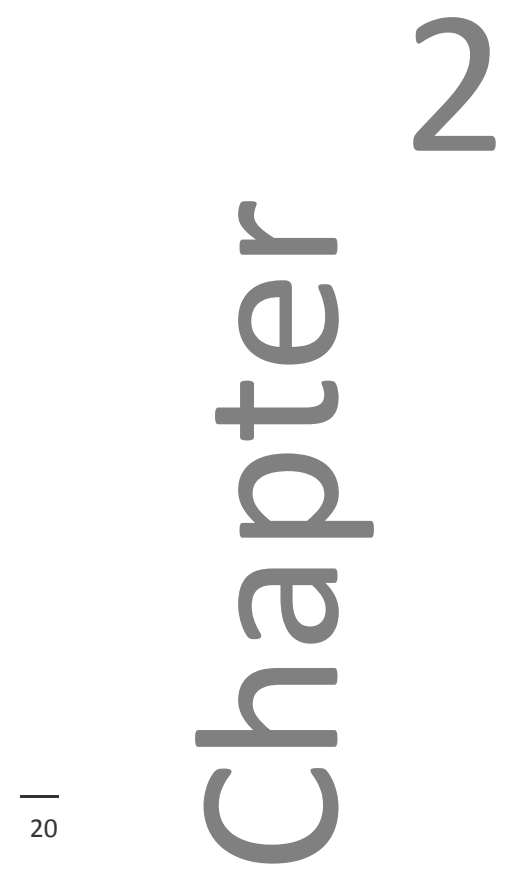
Explorative studies in humans on techniques with targeted delivery of substrates to the distal gut 


\section{Introduction}

The colon has several important functions but absorption of water, electrolytes and also nutrients is considered to be among the most relevant ones. The presence of a complex microbial ecosystem within the colon contributes to important functions for the host such as colonisation resistance, metabolic processing of nutrients, production of vitamins and also the host immune system is affected. Studying human colon metabolism and function in vivo is challenging due to its limited accessibility. Scientific interest in local drug or nutrient delivery and in devices to sample luminal contents is mainly driven by the need to locally administer drugs in the colon for diseases such as Inflammatory Bowel Disease (IBD) or colorectal cancer. Interest in the composition of colonic microbiota and its metabolic capacity is expanding, as intestinal bacteria and short chain fatty acids (SCFA), mainly propionate, acetate and butyrate, produced by microbial fermentation of non-digestible carbohydrates, are found to play a key role in both maintaining and restoring gut health ${ }^{1}$. Butyrate, for example, has been shown to be an important energy source for colonocytes ${ }^{2}$ and affects a wide variety of processes in the human colon ${ }^{3}$. Butyrate is known to increase colonic barrier function and anti-oxidant capacity, to decrease inflammation and it has anti-carcinogenic potential ${ }^{1}$. Many of the studies on butyrate have been conducted in vitro or in animals, not in vivo in humans.

Studying the effects of drugs, food derived compounds, bacteria, and bacterial metabolites in vivo in humans, has been hampered by the available tools for colonspecific delivery. Such tools should not require prior bowel cleansing, since this affects the microbial community, induces fluid fluxes and most likely induces local stress and hence may affect colonic function and mucosal gene expression.

Enemas $^{4,5}$, catheters ${ }^{6-10}$, remote controlled capsules $^{11-16}$ and enteric coated capsules have previously been developed for colon specific delivery in scientific intervention studies as well as for drug delivery. Since we were interested in the effects of microbial metabolites (i.e. butyrate) in the distal human intestine, we evaluated the delivery of substrates to a) the proximal colon by enteric-coated capsules, b) to the distal ileum by an oro-ileal catheter and c) to the distal colon by rectal enemas in three separate explorative studies. The knowledge obtained with these experiments has been used in both the chapters on rectal delivery of substrates with enemas and in further projects with ileal intubation performed at our department. 


\section{Explorative study A: Substrate delivery to the proximal colon by enteric-coated capsules}

\section{Introduction}

A variety of enteric coated capsules for targeted delivery is currently available. The underlying technology of different coatings for colon delivery is mostly based on pressure-triggered ${ }^{14}, \mathrm{pH}$-triggered $^{14,17-19}$, time-released ${ }^{14,20}$ or microbially degradable coatings $^{14,21,22}$, each item with their own limitations ${ }^{14}$. The time-released capsules, for example, may vary in opening location due to variation in transit time between subjects, whereas the $\mathrm{pH}$ sensitive coatings may fail to disintegrate due to interindividual differences in intra-luminal $\mathrm{pH}$ of the colon, which is influenced by diet and microbial composition. The $\mathrm{pH}$ sensitive coating Eudragit ${ }^{\circledR} \mathrm{FS} 30 \mathrm{D}$ has been proven to be reliable to target the terminal ileum / proximal colon in healthy volunteers when applied and manufactured traditionally ${ }^{18,19,23,24}$. However, precoating of capsules prior to filling will facilitate the application in human intervention studies, since the precoated capsules can be filled and manufactured by local hospital pharmacies. Huyghebaert et al. ${ }^{18}$ validated in vitro a new protocol to precoat bodies and caps that can easily be filled prior to application without specific equipment. Accurate targeted delivery of capsule content is crucial. The aim of our explorative study was to evaluate the location of opening of capsules pre-coated with the $\mathrm{pH}$-triggered coating Eudragit FS30D for substrate delivery in the terminal ileum or proximal colon in vivo, using $\gamma$ scintigraphy for monitoring and identification of the capsule disintegration.

\section{Materials and methods}

\section{Subjects}

Seven healthy subjects (5 males, 2 females; mean age 30 \pm 15 years, mean \pm SD) participated in this explorative study. None of them used medication nor had a history of gastrointestinal or metabolic diseases or previous abdominal surgery. This study was approved by the Medical Ethics Committee of the Maastricht University Medical Center (MUMC) and all volunteers gave their written informed consent prior to participation.

\section{Preparation and filling of the capsules}

The protocol used for the pre-coating of the capsules is identical to the one described by Huyghebaert et al. ${ }^{18}$. Briefly, $2.3 \mathrm{~g}$ of triethyl citrate (plasticiser) (Sigma-Aldrich, Bornem, Belgium), $2.3 \mathrm{~g}$ of a polysorbate solution (33\% v/w) (wetting agent) 
(Tween 80, Alpha pharma, Nazareth, Belgium) and 1.9g of glycerol monostearate (glidant)(Federa, Braine-l'Alleud, Belgium) were added to $103.3 \mathrm{~g}$ water and stirred over a period of $10 \mathrm{~min}$ with a high-speed mixer (Silverson, Bucks, UK) until a homogenous dispersion was obtained. This dispersion was gently added to $77.1 \mathrm{~g}$ of Eudragit ${ }^{\circ}$ FS30D dispersion $(30 \%, v / w)$. The coating dispersion was then passed through a $0.3 \mathrm{~mm}$ sieve and stirred continuously using a magnetic stirrer.

Per batch 650 Hydroxypropyl Methylcellulose (HPMC) caps or $700 \mathrm{ml}$ bodies 00 (Vcaps) (total surface area of $0.2 \mathrm{~m}^{2}$ ) were coated separately in a fluid bed apparatus (GPCG-1, Glatt, Binzen, Germany) using the bottom spray mode with Wurster set-up (E.D. Wurster, US Pat. 3,196,827, 1965). All capsules were coated with $10 \mathrm{mg}$ polymer $/ \mathrm{cm}^{2}$ and stored at room temperature and low relative humidity $(20 \% \mathrm{RH})$. Capsules can be stored under these conditions for at least 6 months ${ }^{18}$.

Capsule bodies were filled with radioactively labelled Indium $\left({ }^{111} \mathrm{In}, 3.7 \mathrm{MBq}\right)$ by the local department of nuclear medicine (in accordance to the Dutch legislation and permits of the MUMC) in the morning of the test day and closed manually by gently sliding on the coated caps.

\section{Test day}

Subjects arrived at the MUMC at $8.00 \mathrm{AM}$ after an overnight fast. The first two volunteers (one male and one female) ingested 3 coated capsules containing ${ }^{111}$ In, with $200 \mathrm{ml}$ of water. Thereafter, the five other volunteers ingested only 2 instead of three capsules to be able to better evaluate capsule disintegration. After oral ingestion, the volunteers were allowed to move freely but had to abstain from food intake for the first five hours, but water was permitted ad libitum. Five hours after ingestion, the volunteers received a standardised lunch (i.e. one sandwich with ham and cheese). At regular hourly intervals, starting 10 minutes after ingestion, a gamma camera recording was performed until 5.00 PM to monitor the passage of the capsules through the gastrointestinal tract and to observe time and location of capsule disintegration. The next morning (at $T \approx 24$ hour), a final recording was performed at the MUMC. At this time point, the labelled indium had dispersed through the entire colon, which allows obtaining an anatomical overview of the colon. In order to enable comparison between the different gamma recordings within one subject, a technetium marker was placed on the volunteer's umbilicus. Furthermore, this marker was used, together with the last recording, to draw the contours of the colon, used to define the anatomical location of the capsules (Figure 2.1). All recordings were evaluated independently by a radiologist, a gastroenterologist and the researcher in order to determine capsule disintegration (time, location) in each volunteer. The individual findings were combined and discussed to obtain final agreement on the exact location of capsule disintegration. 


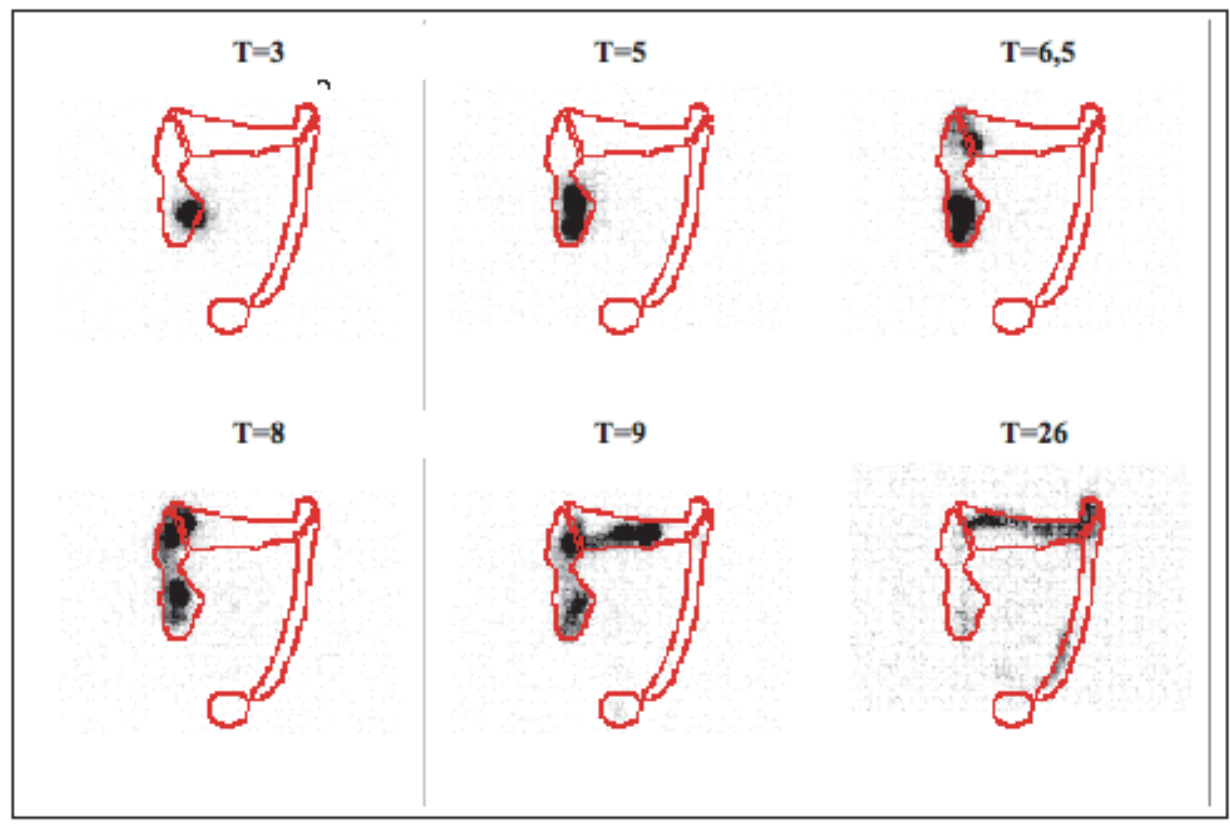

Figure 2.1 This figure shows Gamma camera recordings of ingested capsules containing 111 In in one representative volunteer at consecutive time points during the day ( $T$ in hours after ingestion). The spread of radioactively labeled Indium was used to draw the contours of the colon. The umbilical marker was used to overlay the contours on all consecutive recordings.

\section{Results}

From the 16 capsules, ingested by 7 subjects ( 5 males, mean age $30 \pm 15$ years), 11 capsules opened in the terminal ileum and 2 in the proximal colon. The remaining three capsules opened in the transverse or descending colon and were all ingested by the same volunteer. None of the tested capsules opened proximal to the terminal ileum (Figure 2.1 and 2.2). 


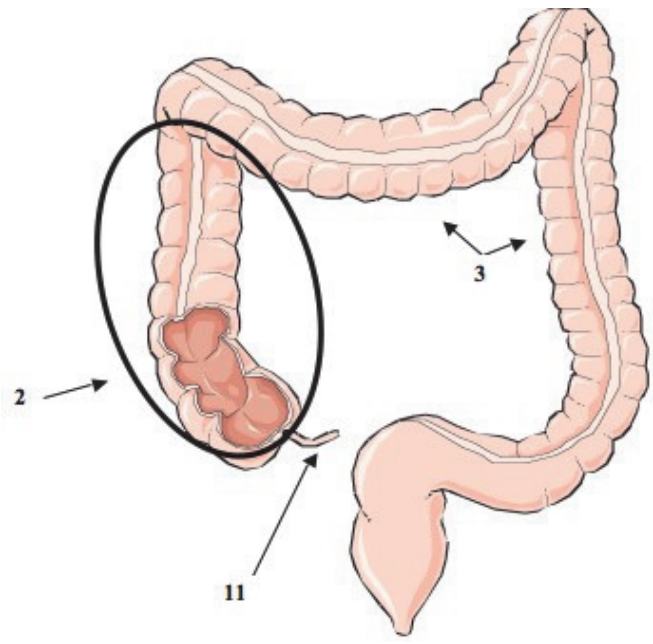

Figure 2.2 This figure shows the targeted area and the number of capsules that opened at the specific location. Target area is indicated by the oval. Opening locations are indicated by arrows.

\section{Discussion}

The precoated capsules used in this study were shown to all disintegrate in the terminal ileum or proximal colon in six out of seven volunteers.

In healthy volunteers intraluminal $\mathrm{pH}$ will rise gradually from 1.0-5.0 in the stomach, 6.5 in the duodenum to 7.5 in the terminal ileum. In the cecum pH drops slightly and rises again to approximately 7 in the distal colon $^{25}$. The Eudragit ${ }^{\circ}$ FS30D coated capsules disintegrate at $\mathrm{pH} 7.2$, which is expected to be present in the terminal ileum. Three capsules taken by one volunteer, did not disintegrate at the target area (i.e. terminal ileum). The observation that in one subject, capsule disintegrated distal to the target area, suggests that individual $\mathrm{pH}$ profile along the gut may result in unsuccessfully targeting the terminal ileum. Although the number of subjects in the present study is very small, it is to be expected that the capsules will disintegrate either proximal and prior to or distal to the target location in a subset of subjects. In our study in $15 \%$ of subjects due to inter-individual differences for instance in intestinal $\mathrm{pH}$. The gastrointestinal $\mathrm{pH}$ profile is influenced by diet, by intestinal microbiota composition and microbial metabolic activity, such as the production of $\mathrm{SCFA}^{26}$. Also the onset of an inter-digestive migrating motor complex (MMC) may have contributed to the aberrant disintegration of the $\mathrm{pH}$-sensitive capsules. This MMC may have resulted in rapid transit of the capsules through the small intestine to 
terminal ileum with delivery into the cecum and colon where capsule disintegration may be delayed due to the drop in $\mathrm{pH}$.

Although the capsules opened at the target location in the majority of subjects, the delayed opening in one out of 7 subjects emphasizes the importance of controlling accurate capsule disintegration in human intervention studies where targeting the delivery at a specific location is crucial. The present study was performed in healthy volunteers. Whether similar results can be obtained in specific patient groups needs further evaluation. For example, lower intestinal $\mathrm{pH}$ levels have been reported in patients with ulcerative colitis or Crohn's disease and thus may affect capsule desintegration in IBD differently compared to healthy voluteers ${ }^{27,28}$

In the present study, $\gamma$-scintigraphy was used to monitor capsule disintegration. A major drawback of this elegant technique is the need for hospital facilities with gamma camera recording. Monitoring capsule opening is therefore limited to dedicated intervention studies. In the present study, intermittent recordings were taken at regular, hourly intervals which may have resulted in missing the specific time and place of capsule disintegration in some subjects. Verbeke et al. ${ }^{29}$ used stable isotope techniques enabling continuous monitoring without the potential harm of radiation exposure and injury. In that study, labelled compounds $\left({ }^{13} \mathrm{C},{ }^{15} \mathrm{~N}\right.$ and Raftilin $\mathrm{HP}$ ) were measured in breath or urine to determine orocecal transit time and to discriminate between disintegration of capsules in colon or ileum. However, this technique does not discriminate between the numbers of capsules that disintegrate at each location and does not provide information about the exact opening location. Another possible drawback for the use of capsules, especially for delivering (functional) food ingredients is their small capacity and volume. In the present study, capsules with a volume of approximately $1 \mathrm{ml}$ were used. The number of capsules required to deliver for example an amount of butyrate comparable to that delivered by with a $60 \mathrm{ml}$ enema containing $100 \mathrm{mM}$ of butyrate is too high to be practically feasible.

In conclusion, pre-coated $\mathrm{pH}$ sensitive capsules can be applied for distal ileal or proximal colonic delivery of substrates in vivo. However, in $15 \%$ of subjects the capsules did not disintegrate at the intended location but beyond the proximal colon. This subject could not be differentiated from other subjects based on clinical characteristics or bowel habits. Therefore variability is unpredictable and too high to be employed for scientific purposes without use of markers and scintigraphy. The technique needs further optimisation and evaluation. In addition, the limited volume that can be retained in capsules may hamper its potential clinical applications. 


\section{Explorative study B: Substrate delivery to the distal ileum by use of an oro-ileal catheter}

\section{Introduction}

Gastrointestinal catheters provide the opportunity to deliver compounds to different pre-defined locations in the gastrointestinal tract, with the advantage of the option of

simultaneously samping of luminal contents ${ }^{6-10,30,31}$. Depending on the aim and target area, the catheters may vary in length and can be introduced either nasally, orally or rectally. Orally and nasally introduced catheters can be placed intragastically without the need for imaging or tracking devices. For prolonged application or use, nasally catheters are preferred over orally introduced catheters due to the lower burden to patients or subjects. Positioning at more distal locations will be facilitated by natural peristalsis and interdigestive motility, but requires imaging techniques, such as x-ray imaging of radio-opaque markers on the catheter. Rectally introduced catheters need to be positioned by endoscopy and fixed with balloons or endoclips to prevent displacement of the catheter because of peristalsis and aboral passage of luminal contents.

We aimed to optimise an existing oro-ileal catheter for substrate delivery in the distal ileum and tested its positioning by normal peristaltic movements in healthy subjects. Special attention was paid to feasibility of sampling of luminal contents and retraction of the catheter.

\section{Materials and methods}

\section{Subjects}

Four healthy non-smoking subjects ( 2 male/ 2 female, aged $30 \pm 8.5$ years old, BMI $18-25 \mathrm{~kg} / \mathrm{m} 2$ ) and no history of gastrointestinal disorders, surgery or diabetes type 1 or 2, participated in this pilot study. None of them had received antibiotic treatment for at least 2 months prior to inclusion, nor had a history of current or prior use of drugs. All volunteers gave their written informed consent prior to participation. The study was approved by the Medical Ethics Committee of the Maastricht University Medical Center (MUMC).

\section{Catheter}

A 2.5 meter catheter was constructed from a 13-lumen polyvinyl tubing with an outer diameter of $4.6 \mathrm{~mm}$. The catheter consisted of a large central lumen with a diameter of $2.2 \mathrm{~mm}$, surrounded by 12 small lumina with an inner diameter of $0.4 \mathrm{~mm}$ each. 
(CMPT14FR13LUM; Mui Scientific, Ontario, Canada). At the tip, an inflatable latex balloon was attached with a maximum volume of $10 \mathrm{ml}$. The infusion port was located $11 \mathrm{~cm}$ proximal to the balloon and was connected to two of the small lumina. The sampling port was connected to the large central lumen and was located $1 \mathrm{~cm}$ proximal to the balloon. The balloon itself was connected to one of the small lumina to enable inflation and deflation of the balloon. The other 9 lumina were closed. The large central lumen of the catheter was used to sample luminal contents and to introduce a radio opaque guide wire (Figure 2.3).

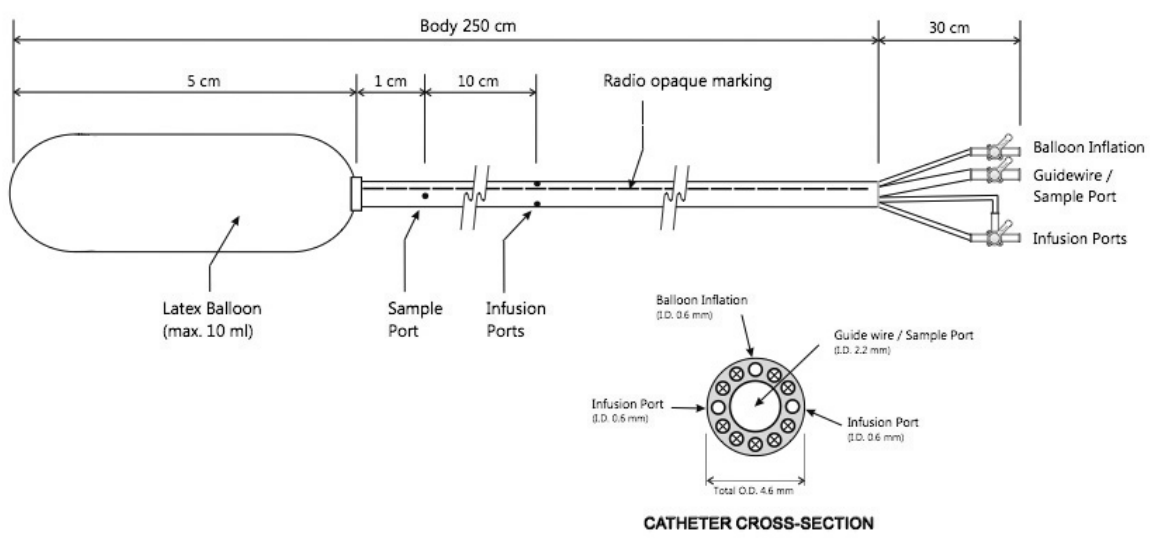

Figure 2.3 This is a schematic drawing of the catheter used in this pilot experiment. A 13- lumen catheter, with a balloon attached to the tip. The large central lumen was used for luminal sampling whereas 2 of the small lumina were used for substrate infusion. One small lumen was used for balloon inflation. The 9 other lumina were closed.

\section{Protocol (Test day)}

The subjects $(n=4)$ arrived at at $13.00 \mathrm{~h}$ on day 1 in MUMC and stayed overnight until the end of the experiment. The four subjects did undergo the positioning on separate days.

After arrival, the catheter was introduced orally or nasally and positioned through the pylorus using a positioning wire by an experienced gastroenterologist. The position of the tip of the catheter was checked fluoroscopically. When the tip was placed and positioned beyond the descending duodenum, the positioning wire was withdrawn and the balloon was inflated with $10 \mathrm{ml}$ of air. The subsequent positioning of the catheter was facilitated by the intestinal peristalsis. After $45 \mathrm{~min}$, the position was checked again by fluoroscopy.

At 7 PM, a standardised meal was served, followed by a snack at 9 PM. Further positioning of the catheter was allowed overnight. The balloon attached at the tip was deflated and inflated every $20 \mathrm{~min}$, to allow intestinal passage by peristalsis without 
prolonged distension with risk of ischaemia. After an overnight positioning period, the location of the catheter tip, was checked again fluoroscopically and a standardised breakfast was given when the catheter did not reach the final position yet. When the catheter tip was located in the terminal ileum (proximal to the cecum), the balloon was deflated completely to prevent the catheter from moving beyond the cecum.

At the end of the test day, a gastroenterologist gently pulled out the catheter after intravenous infusion of $1.0 \mathrm{ml}$ of Buscopan ${ }^{\circledR} 20 \mathrm{mg} / \mathrm{ml}$ (butylscopalamine bromide for intramuscular or intravenous injection, Registration number for medication: RVG 03837, Boehringer Ingelheim, Alkmaar, The Netherlands).

\section{Sampling of luminal contents}

After succesfull positioning, retraction and substrate administration in the first two volunteers, the experimental setting was extended in subject 3 and 4 in which we succeeded to sample luminal contents from the terminal ileum.

The analysis of the samples was beyond the scope of this study.

\section{Results}

Based on the fluoroscopic images the catheter positioning in the terminal ileum was successful in all four subjects. To illustrate the anatomical structures with the catheter in place, Figure 2.4 represents a fluoroscopic image of one volunteer, indicating the positioning of the catheter tip entering the proximal colon. In this particular case, intestinal structures were visualised for fluoroscopic imaging by administering $10 \mathrm{ml}$ of a solution containing Lipiodol ${ }^{\circledR}$ Ultra Fluide $480 \mathrm{mg} \mathrm{Jood} / \mathrm{ml}$ (Guerbet Nederland BV, Gorinchem, The Netherlands). The Lipiodol ${ }^{\circledR}$ was diluted in saline $(0.9 \% \mathrm{NaCl})$ in a ratio of 1:3. In the experiments described here, the catheter was placed with the tip positioned in the terminal ileum without the use of contrast fluid.

Two subjects suffered from bowel cramps and in one the catheter had to be retracted immediately after successful catheter positioning and the intestinal perfusion. In the other subject the abdominal symptoms readily disappeared after catheter removal.

In all experiments the intestinal perfusion proved to be successful. Sampling of the luminal content $(n=2)$ was successful at all time points $(0 h, 0.5 h, 1 h, 2 h, 3 h$ and $4 h$ after infusion). 


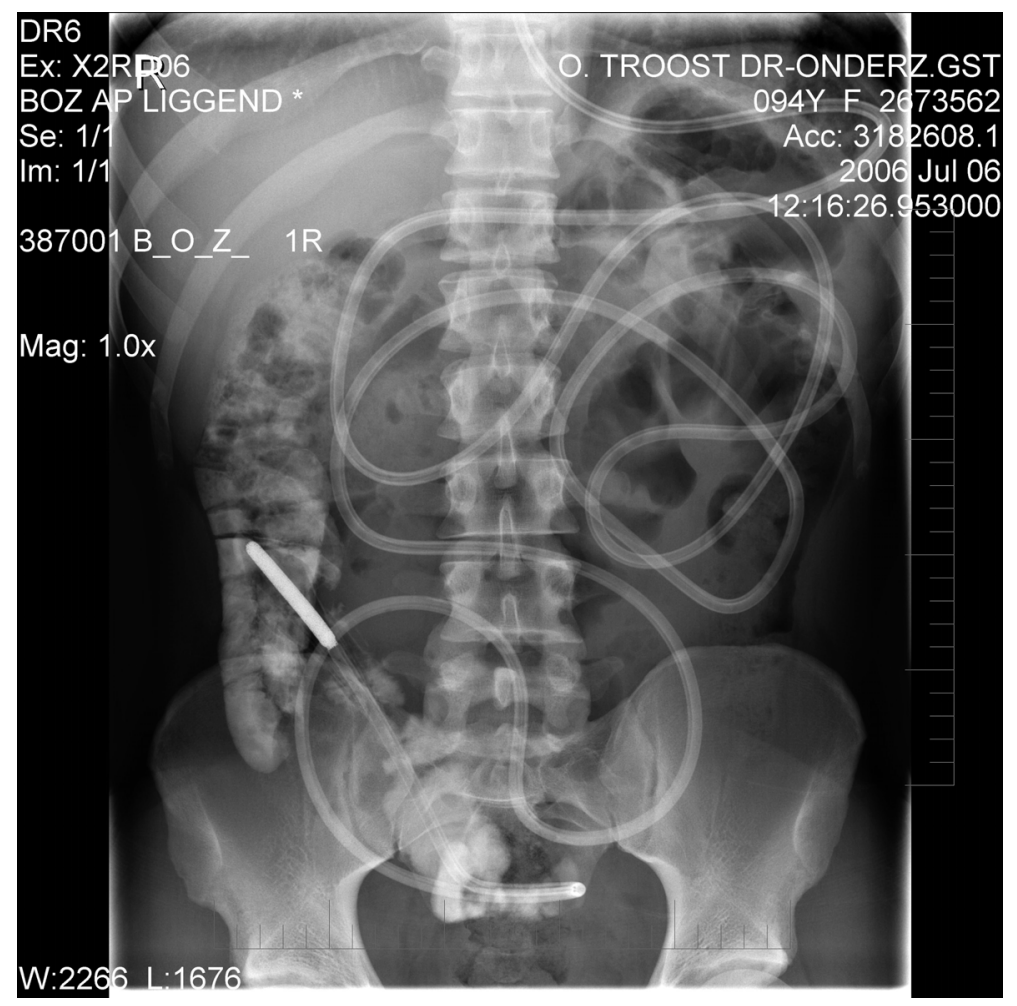

Figure 2.4 Fluoroscopic image of one volunteer, indicating the positioning of the catheter tip entering the proximal colon. Especially for this picture, intestinal structures were visualised for fluoroscopic imaging by administering $10 \mathrm{ml}$ of a solution containing Lipiodol ${ }^{\circledR}$ Ultra Fluide $480 \mathrm{mg} \mathrm{Jood} / \mathrm{ml}$ (Guerbet Nederland BV, Gorinchem, The Netherlands). The Lipiodol ${ }^{\circledR}$ was diluted in saline $(0.9 \% \mathrm{NaCl})$ in a ratio of $1: 3$

\section{Discussion}

The ileum catheter we designed proved to be a successful tool for delivery of substances into the terminal ileum and sampling from the terminal ileum in vivo. Although this method is rather invasive and time consuming, it is the only technique that allows sampling of intraluminal gut contents. The flexibility of the catheter and total deflation of the balloon were found to be crucial elements for a successful positioning and also retraction of the ileum catheter. Stiff catheters or residual air in the balloon leads to patient discomfort and early discontinuation of the experiment. Furthermore, use of hyoscine butylbromide (Buscopan), which is a smooth muscle relaxant, for the retraction of the catheter was found to be helpful. 
To minimise the disturbance of the physiological conditions in the bowel, the catheter position was checked fluoroscopically without the use of contrast agent or air, in order not to interfere with the colonic physiology. However, the exact location of the catheter tip in relation to the cecal valve was difficult to determine. Localisation of the position of the catheter in relation to the cecal valve should be optimised. Here we advice the use of visualisation tools not interfering with the colonic function. In this explorative study adequate positioning of the tip of the catheter was obtained, catheters could always be retracted and luminal samples from the terminal ileum were retrieved successfully.

In conclusion, using a multi-lumen long catheter, it is feasible to access the human lower intestinal tract for administering study substances and/or sampling of luminal contents. This explorative study has shown that application of oro-ileal intubation techniques is feasible albeit with considerable effort and test person discomfort. 


\section{Explorative study C: Substrate delivery to the distal colon by rectal enemas}

\section{Introduction}

Enemas are widely used as tool for drug delivery in the distal colon in a clinical setting. The retrograde spread throughout the colon has been tested extensively in patients with $\mathrm{IBD}^{4,5,32-34}$. Since gastrointestinal motility, stool consistency and mucosal secretion rates of water and mucus may vary between patients suffering from gastrointestinal disorders, both speed and extent of retrograde spread might vary between patients with gastrointestinal disorders and healthy subjects. Only few studies have evaluated retrograde spread of enemas in healthy volunteers. Otten et al. reported the retrogade spread of two types of 5-ASA enemas with different volumes (30 and 50 $\mathrm{ml}$, respectively) in healthy volunteers after a distal bowel preparation with sodium phosphate ${ }^{35}$. They reported a maximum spread of $38 \mathrm{~cm}$ versus $23 \mathrm{~cm}$ by the $50 \mathrm{ml}$ enema versus the $30 \mathrm{ml}$ enema, respectively. Bowel preparation may affect not only enema spread but also colonic physiology. Therefore, proximal enema spread should also be evaluated in an unprepared colon (i.e. without prior bowel cleansing). One study in healthy volunteers reported a maximum spread of a $100 \mathrm{ml}$ enema up to the transverse colon without prior cleansing but the authors found a relatively large variation in both maximum spread and retention time ${ }^{36}$. Data on the target area of smaller volume enemas in an unprepared colon for healthy volunteers are currently not available.

The aim of the present explorative study was to evaluate the retrograde spread of a test solution, administered via a small enema $(60 \mathrm{ml})$ into the distal colon in healthy volunteers in an unprepared colon, that is without prior bowel cleansing. This was performed as explorative study to obtain data for future human intervention studies with butyrate enemas. The target location of the enema content was the rectum and midsection of the sigmoid colon.

\section{Materials and methods}

\section{Subjects}

Two healthy subjects (both female, age: 20 and 22 resp.), without a history of gastrointestinal or metabolic diseases or previous abdominal surgery participated in this explorative study. Exclusion criteria were the use of pre- or probiotics, the use of medication (other than contraceptives), the use of drugs or alcohol and pregnancy. 
This study was approved by the Medical Ethics Committee of the Maastricht University Medical Center (MUMC) and all volunteers gave their written informed consent prior to participation.

\section{Test day}

Subjects arrived at MUMC at 8:00 AM after an overnight fast. The subjects were instructed to void their rectal contents and take position on the bed in a left lateral position. Subsequently, they self- administered a $60 \mathrm{ml}$ enema with an isotonic saline solution $(0.9 \% \mathrm{NaCl})$ containing radioactively labeled indium $\left({ }^{111} \mathrm{In} ; 3.7 \mathrm{MBq}\right)$. The enemas were prepared by the hospital pharmacy and the department of nuclear medicine according to the guidelines and legislation for processing radioactively labelled material.

After instillation, subjects stayed in a left-lateral position for at least 15 minutes. During the test day, subjects remained in bed except for a short visit to the toilet if necessary. A light standardised lunch consisting of one sandwich with ham or cheese was provided at noon and water was allowed ad libitum. Gamma camera recordings were performed 15, 30 minutes, 1, 3, 5 and 8 hours after enema instillation.

\section{Results}

In Figures 2.5 and 2.6, the gamma camera recordings of the instilled enemas are shown for subject 1 and 2, respectively. The maximum (i.e most proximal) spread of the enema content occurred 1 hour after enema instillation in volunteer 1 and lasted until approximately 5 hours after instillation. Subject 2 did not succeed in voiding rectal contents prior to the start of the experiment. In this subject, the maximum spread of the indium was observed after 4-5 hours. The enema content reached the entire sigmoid colon in both volunteers. Eight hours after instillation the spread of indium was limited to the rectum again. Twenty-four hours after instillation, all radioactively Indium was cleared from the bowel in both subjects. 


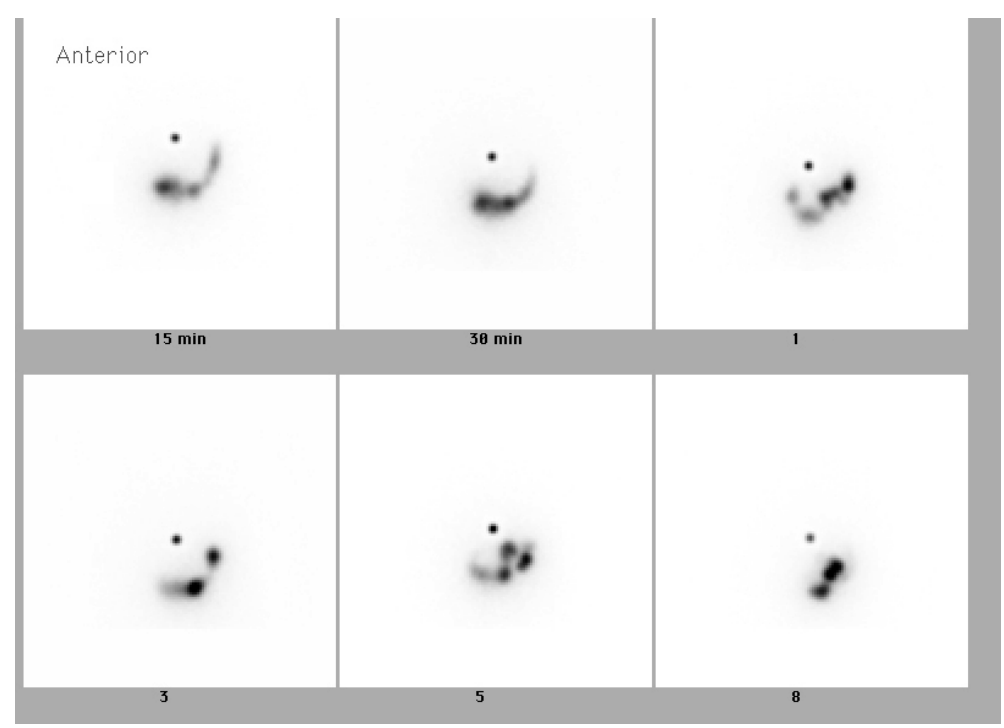

Figure 2.5 This figure shows the consecutive gamma camera recordings for subject 1 . It shows the retrograde spread of a $60 \mathrm{ml}$ enema containing a isotonic solution of $3,7 \mathrm{MBq}{ }^{133} \mathrm{In}$ in saline $(0.9 \% \mathrm{NaCl})$.

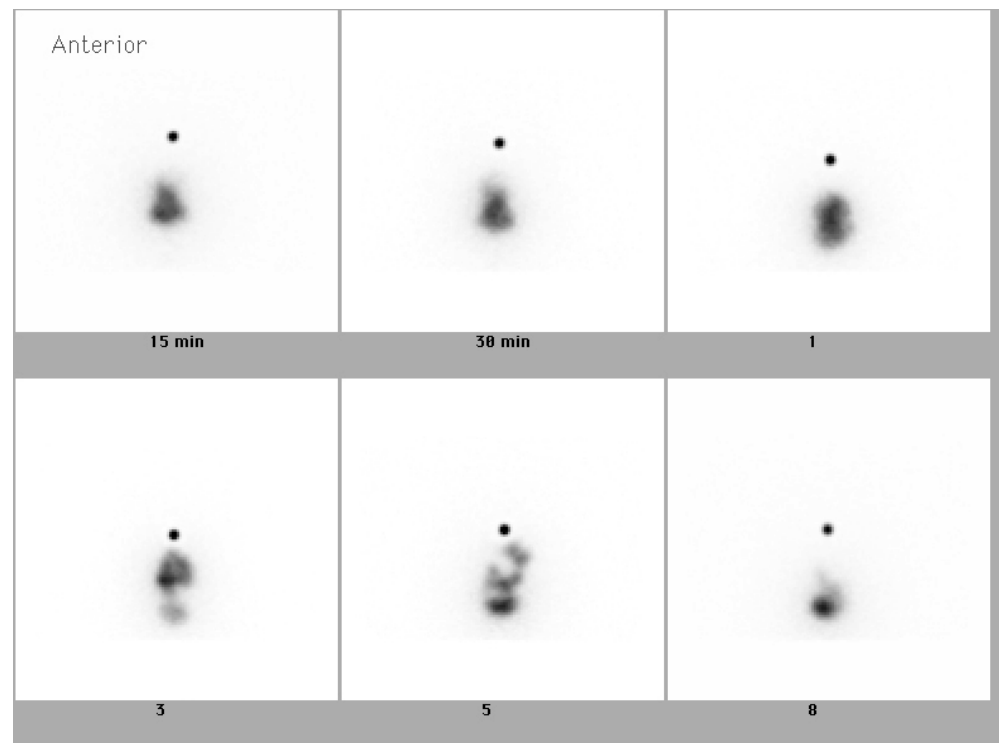

Figure 2.6 This figure shows the consecutive gamma camera recordings for subject 2 . It shows the retrograde spread of a $60 \mathrm{ml}$ enema containing a isotonic solution of $3,7 \mathrm{MBq}{ }^{133} \mathrm{In}$ in saline $(0.9 \% \mathrm{NaCl})$. 


\section{Discussion}

Our results show that in both subjects the enema content reached the entire sigmoid colon but both speed and extent of the spread varied between individuals. The slower spread in one subject may be due to the presence of remaining luminal fecal contents. The lunch that was supplied may have provoked peristaltic activity, negatively affecting retrograde spread of the enemas. Nevertheless, the present explorative study showed that in both subjects the entire sigmoid colon, which was defined as the target location, was reached by the contents of the enema. Furthermore, these results were in line with the study of Brown et al. They reported both a similar variation in the maximum spread between the different subjects $(n=8)$ and time to reach the maximum spread (0.7-4 hours) in a study design that was highly comparable with the present pilot ${ }^{36}$.

During our experiments, the subjects remained in the supine position in bed during the entire 8-hour test period to mimic delivering a substrate just prior to sleep, since this would most likely minimise the risk of anal leakage of the enema contents and maximise the retrograde spread. Further, the use of enemas was found to be relatively easy, successful, and volunteers can self-administer the enemas at home. Moreover, to study the effects of specific components in the colon, the use of enemas also has the advantage that it can deliver relatively large volumes of substances (i.e. 60-100 ml).

In conclusion, the results of the present experiments show that rectal enemas provide a successful tool to deliver relatively large volumes of substances to the sigmoid colon, although the maximum spread and the retention time may vary between subjects.

\section{General conclusion}

Three routes of administrating test substances to the distal intestine (i.e. distal ileum, proximal colon and distal colon, respectively) were evaluated in this series of explorative studies. The use of enteric-coated capsules to target the distal ileum is feasible but only for small volume substrate delivery. Furthermore, the variation in location where the capsules disintegrate requires specific monitoring and may limit its application in large clinical trials. The application of an oro-cecal catheter was found to be feasible. By employing such a catheter, substances can be effectively delivered to the proximal colon, offering the possibility of sampling luminal content. For various purposes, it is a very promising tool but it is rather invasive and time consuming and requires specific expertise. It is therefore only applicable in dedicated centres. Rectal enemas were found to target the rectum and sigmoid colon with substantial precision and have the advantage of delivering relatively large volumes up to $100 \mathrm{ml}$. An 
additional advantage of the use of enemas is that subjects can self-administer the enemas at home without the need of hospital visits.

All three evaluated techniques were found to be adequate for delivering substances to different locations in the distal human intestine in vivo, each with their advantages and disadvantages. Their application thereby depends on the aim of a study. In the studies described in the following chapters of this thesis, we aim to target the sigmoid colon for a prolonged period of time (2-3 weeks) and therefore used rectal enemas. In future intervention studies that will be performed at our department we will use and apply the information obtained in these explorative studies. 


\section{References}

1. Hamer HM, Jonkers D, Venema K, et al. Review article: the role of butyrate on colonic function. Aliment Pharmacol Ther 2008;27:104-19.

2. Topping DL, Clifton PM. Short-chain fatty acids and human colonic function: roles of resistant starch and nonstarch polysaccharides. Physiological reviews 2001;81:1031-64.

3. Vanhoutvin SA, Troost FJ, Hamer HM, et al. Butyrate-induced transcriptional changes in human colonic mucosa. PLoS ONE 2009;4:e6759.

4. Campieri M, Corbelli C, Gionchetti P, et al. Spread and distribution of 5-ASA colonic foam and 5-ASA enema in patients with ulcerative colitis. Dig Dis Sci 1992;37:1890-7.

5. Chapman NJ, Brown ML, Phillips SF, et al. Distribution of mesalamine enemas in patients with active distal ulcerative colitis. Mayo Clin Proc 1992;67:245-8.

6. Lennernas $\mathrm{H}$, Fagerholm $U$, Raab $\mathrm{Y}$, et al. Regional rectal perfusion: a new in vivo approach to study rectal drug absorption in man. Pharm Res 1995;12:426-32.

7. Chauve A, Devroede G, Bastin E. Intraluminal pressures during perfusion of the human colon in situ. Gastroenterology 1976;70:336-40.

8. Raab Y, Hallgren R, Knutson L, et al. A technique for segmental rectal and colonic perfusion in humans. Am J Gastroenterol 1992;87:1453-9.

9. Troost FJ, Saris WH, Haenen GR, et al. New method to study oxidative damage and antioxidants in the human small bowel: effects of iron application. Am J Physiol Gastrointest Liver Physiol 2003;285:G354-9.

10. Troost FJ, Brummer RJ, Haenen GR, et al. Gene expression in human small intestinal mucosa in vivo is mediated by iron-induced oxidative stress. Physiol Genomics 2006;25:242-9.

11. Wikling IR, Prior DV. Remote controlled capsules in human drug absorption (HDA) studies. Crit Rev Ther Drug Carrier Syst 2003;20:405-31.

12. LaVan DA, McGuire T, Langer R. Small-scale systems for in vivo drug delivery. Nat Biotechnol 2003;21:1184-91.

13. Clear NJ, Milton A, Humphrey M, et al. Evaluation of the Intelisite capsule to deliver theophylline and frusemide tablets to the small intestine and colon. Eur J Pharm Sci 2001;13:375-84.

14. Basit AW. Advances in colonic drug delivery. Drugs 2005;65:1991-2007.

15. Wilding I. Site-specific drug delivery in the gastrointestinal tract. Crit Rev Ther Drug Carrier Syst 2000; 17:557-620.

16. Moglia A, Menciassi A, Schurr MO, et al. Wireless capsule endoscopy: from diagnostic devices to multipurpose robotic systems. Biomed Microdevices 2007;9:235-43..

17. Ibekwe VC, Liu F, Fadda HM, et al. An investigation into the in vivo performance variability of $\mathrm{pH}$ responsive polymers for ileo-colonic drug delivery using gamma scintigraphy in humans. J Pharm Sci 2006;95:2760-6.

18. Huyghebaert N, Vermeire A, Remon JP. Alternative method for enteric coating of HPMC capsules resulting in ready-to-use enteric-coated capsules. Eur J Pharm Sci 2004;21:617-23.

19. Cole ET, Scott RA, Connor AL, et al. Enteric coated HPMC capsules designed to achieve intestinal targeting. Int J Pharm 2002;231:83-95.

20. Hebden JM, Wilson CG, Spiller RC, et al. Regional differences in quinine absorption from the undisturbed human colon assessed using a timed release delivery system. Pharm Res 1999;16:108792.

21. Sinha VR, Kumria R. Microbially triggered drug delivery to the colon. Eur J Pharm Sci 2003;18:3-18.

22. Katsuma M, Watanabe S, Takemura S, et al. Scintigraphic evaluation of a novel colon-targeted delivery system (CODES) in healthy volunteers. J Pharm Sci 2004;93:1287-99.

23. Ibekwe VC, Fadda HM, Parsons GE, et al. A comparative in vitro assessment of the drug release performance of $\mathrm{pH}$-responsive polymers for ileo-colonic delivery. Int J Pharm 2006;308:52-60.

24. Huyghebaert N, Vermeire A, Remon JP. In vitro evaluation of coating polymers for enteric coating and human ileal targeting. Int J Pharm 2005;298:26-37.

25. Sasaki $\mathrm{Y}$, Hada R, Nakajima $\mathrm{H}$, et al. Improved localizing method of radiopill in measurement of entire gastrointestinal $\mathrm{pH}$ profiles: colonic luminal $\mathrm{pH}$ in normal subjects and patients with Crohn's disease. Am J Gastroenterol 1997;92:114-8. 
26. Scheppach W, Luehrs H, Menzel T. Beneficial health effects of low-digestible carbohydrate consumption. Br J Nutr 2001;85 Suppl 1:S23-30.

27. Fallingborg J, Christensen LA, Jacobsen BA, et al. Very low intraluminal colonic pH in patients with active ulcerative colitis. Dig Dis Sci 1993;38:1989-93.

28. Nugent SG, Kumar D, Rampton DS, et al. Intestinal luminal pH in inflammatory bowel disease: possible determinants and implications for therapy with aminosalicylates and other drugs. Gut 2001;48:571-7.

29. Verbeke K, de Preter V, Geboes K, et al. In vivo evaluation of a colonic delivery system using isotope techniques. Aliment Pharmacol Ther 2005;21:187-94.

30. Maljaars J, Romeyn EA, Haddeman E, et al. Effect of fat saturation on satiety, hormone release, and food intake. Am J Clin Nutr 2009;89:1019-24.

31. Maljaars PW, Symersky T, Kee BC, et al. Effect of ileal fat perfusion on satiety and hormone release in healthy volunteers. International journal of obesity (2005) 2008;32:1633-9.

32. Vitti RA, Meyers F, Knight LC, et al. Quantitative distribution of radiolabeled 5-aminosalicylic acid enemas in patients with left-sided ulcerative colitis. Dig Dis Sci 1989;34:1792-7.

33. Kruis W, Bull U, Eisenburg J, et al. Retrograde colonic spread of sulphasalazine enemas. Scand J Gastroenterol 1982;17:933-8.

34. Palmer KR, Goepel JR, Holdsworth CD. Sulphasalazine retention enemas in ulcerative colitis: a doubleblind trial. Br Med J (Clin Res Ed) 1981;282:1571-3.

35. Otten MH, De Haas G, Van den Ende R. Colonic spread of 5-ASA enemas in healthy individuals, with a comparison of their physical and chemical characteristics. Aliment Pharmacol Ther 1997;11:693-7.

36. Brown J, Haines S, Wilding IR. Colonic spread of three rectally administered mesalazine (Pentasa) dosage forms in healthy volunteers as assessed by gamma scintigraphy. Aliment Pharmacol Ther 1997;11:685-91. 


$$
3
$$

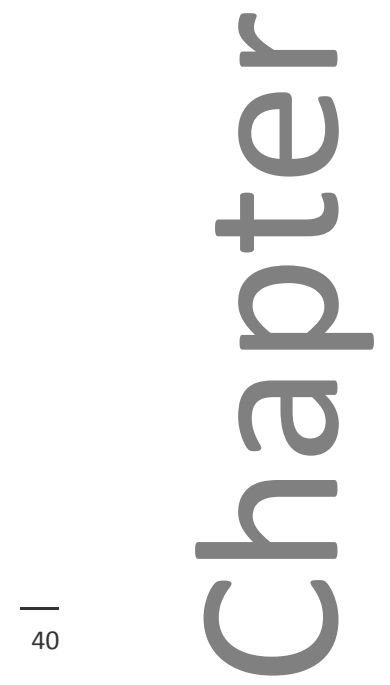




\section{Butyrate-induced transcriptional changes in human colonic mucosa}

Steven ALW Vanhoutvin, Freddy J Troost, Henrike M Hamer, Patrick J Lindsey, Ger H Koek, Daisy MAE Jonkers, Andrea Kodde, Koen Venema, Robert JM Brummer PLoS One. 2009;4:e6759 


\section{Abstract}

\section{Background}

Fermentation of dietary fiber in the colon results in the production of short chain fatty acids (mainly propionate, butyrate and acetate). Butyrate modulates a wide range of processes, but its mechanism of action is mostly unknown. This study aimed to determine the effects of butyrate on the transcriptional regulation of human colonic mucosa in vivo.

\section{Methodology/Principal Findings}

Five hundred genes were found to be differentially expressed after a two week daily butyrate administration with enemas. Pathway analysis showed that the butyrate intervention mainly resulted in an increased transcriptional regulation of the pathways representing fatty acid oxidation, electron transport chain and oxidative stress. In addition, several genes associated with epithelial integrity and apoptosis, were found to be differentially expressed after the butyrate intervention.

\section{Conclusions/Significance}

Colonic administration of butyrate in concentrations that can be achieved by consumption of a high-fiber diet enhances the maintenance of colonic homeostasis in healthy subjects, by regulating fatty acid metabolism, electron transport and oxidative stress pathways on the transcriptional level and provide for the first time, detailed molecular insight in the transcriptional response of gut mucosa to butyrate. 


\section{Introduction}

Short-chain fatty acids (SCFAs) are derived from the microbial fermentation of undigested dietary fibers in the colon. As micro-organisms preferably ferment carbohydrates, most saccharolytic fermentation occurs in the proximal colon. Depletion of carbohydrate sources in the distal colon leads to a switch from saccharolytic to proteolytic fermentation, which is less favorable due to the formation of potentially toxic products. Both these toxic products and the lower availability of SCFAs in the distal colon are hypothesised to be involved in the pathogenesis of gastro-intestinal disorders such as ulcerative colitis (UC) and cancer ${ }^{1-3}$. The amount of SCFAs (mainly acetate, propionate and butyrate) produced in the colon depends on the site of fermentation, the diet and the composition of the microbiota, and can account for up to $5-15 \%$ of the total energy requirements of humans ${ }^{4}$. Fecal concentrations of acetate, propionate and butyrate are found in a molar ratio of approximately $60: 20: 20^{5,6}$, but limited data about luminal concentrations in specific parts of the colon are only available from sudden death patients. Due to rapid absorption and metabolism, actual concentrations may differ. Among the different SCFAs, butyrate is known to modulate numerous processes. It induces cell differentiation and strongly inhibits cell proliferation in tumor cell lines ${ }^{7-13}$. Colonocytes use butyrate as their primary energy source and in the absence of butyrate they undergo apoptosis, but opposite effects were seen in transformed cells, suggesting a possible anti-carcinogenic effect of butyrate ${ }^{13-15}$. Furthermore, butyrate may have an effect on inflammation ${ }^{13}$, oxidative stress ${ }^{13}$, intestinal barrier function ${ }^{13,16,17}$, visceral perception and rectal compliance ${ }^{18}$ and may play a role in satiety $^{19,20}$.

Transcriptional responses of butyrate were studied mostly in cell lines ${ }^{14,15,21-32}$ and some studies were performed in animals and human patients ${ }^{3,30,33-35}$. In vitro and animal studies showed that butyrate downregulates the expression of genes associated with proliferation and oxidative stress and upregulates the expression of Mucin associated genes (Muc 1-4), tight junction proteins (zonulin and occludin) and the butyrate transporter monocarboxylate transporter-1 (MCT1). In UC patients, butyrate was shown to increase the expression of the butyrate transporter MCT-1 and to decrease inflammation by inhibition of the activation of NF- $\kappa \mathrm{B}$. Effects of butyrate on global, genome-wide transcriptional responses of human intestinal mucosa were not described previously.

The aim of this study was to determine the in vivo genome-wide transcriptional response to local administration of butyrate in the distal colon in healthy volunteers in order to identify the biological processes mediated by butyrate, providing new leads for clinical and mechanistic studies. 


\section{Materials and methods}

\section{Objectives}

To determine the in vivo transcriptional response of a local administration of butyrate in the distal colon in healthy volunteers

\section{Participants}

Sixteen healthy volunteers (12 females and 4 males, 18 to 62 years) participated in this study. Exclusion criteria were signs of bowel dysfunction, gastrointestinal surgery, age over 65 years, or use of any medication, probiotics or prebiotics three months prior to inclusion, were excluded from participation. All participants signed an informed consent prior to participation to the study, which was approved by the Ethical Committee of the University Hospital Maastricht, the Netherlands, and conducted in full accordance with the principles of the 'Declaration of Helsinki' $\left(52^{\text {nd }}\right.$ WMA General assembly, Edinburgh, Scotland, Oct 2000). The study has been registered in the US National Library of Medicine (http://www.clinicaltrials.gov) with reference code NCT00693355.

\section{Design}

The study was performed according to a double-blind randomised placebo-controlled cross-over design. The protocol comprised of two experimental periods of two weeks each with a wash-out period of two weeks in between (Figure 3.1). During each experimental period, the subjects self-administered an enema containing $100 \mathrm{mM}$ of butyrate or placebo $(60 \mathrm{ml}, \mathrm{pH} 7.0)$, respectively, once daily prior to sleeping. The local hospital pharmacy department prepared all enemas. The volunteers were asked to defecate, if possible, prior to instillation of the enema. At the end of each experimental period, a sigmoidoscopy was performed in the morning after an overnight fast and biopsy samples were taken from a standardised location in the sigmoid colon (approx. $20 \mathrm{~cm}$ from the anal sphincter at the location of the internal iliac artery). All sigmoidoscopy procedures were performed in an unprepared colon to exclude possible effects induced by the colon cleansing procedure. The diet was standardised 3 days prior to the sigmoidoscopy. After sampling, biopsies were snapfrozen immediately in liquid nitrogen and stored at $-80^{\circ} \mathrm{C}$ until further analysis. 


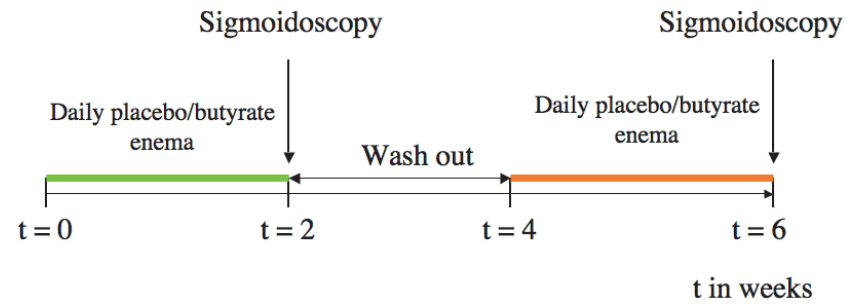

Figure3.1 Design of butyrate study in healthy volunteers.

\section{RNA extraction}

RNA was isolated from frozen biopsies by adding a mixture of $1 \mathrm{ml}$ Trizol (Invitrogen, Carlsbad, USA) and $10 \mu \mathrm{l} \beta$-mercaptoethanol, preheated up to $37^{\circ} \mathrm{C}$. These mixtures were shaken for 30 seconds at maximum speed using a minibeadbeater. $200 \mu \mathrm{l}$ Chloroform was added and after 3 minutes of incubation, the samples were centrifuged for 15 minutes, $21000 \mathrm{~g}$ at $4{ }^{\circ} \mathrm{C} .500 \mu \mathrm{l}$ was taken from the upper colorless phase and mixed with $500 \mu \mathrm{l} 70 \%$ ethanol. RNA was further purified with an RNeasy mini kit (Qiagen, Venlo, The Netherlands) combined with a DNase treatment using the RNase-Free DNase set (Qiagen, Venlo, The Netherlands) according to manufacturers protocol.

Quantity and purity of the RNA samples was determined using the Nanodrop ND-1000 spectrophotometer (Nanodrop Technologies, Wilmington, USA) and RNA integrity was determined using the Bioanalyzer 2100 (Agilent Technologies, Palo Alto, USA).

\section{Microarray hybridization}

Total RNA (150 ng) was amplified using the two-cycle cDNA synthesis kit (Affymetrix, Santa Clara, USA) in combination with the MEGAscript 77 in vitro transcription system (Ambion). Double-stranded cDNA was biotin labeled with the GeneChip in vitro transcription IVT labeling kit (Affymetrix, Santa Clara, USA).

Following fragmentation, $11 \mu \mathrm{g}$ of biotin-labeled cRNA were hybridised for 16 hour at $45^{\circ} \mathrm{C}$ on Affymetrix Human Genome U133 Plus 2.0 Arrays.

GeneChips were washed and stained in the Affymetrix Inc. Fluidics Station 450 (Affymetrix, Santa Clara, USA) and hybridised. Cyclic RNA was detected using streptavidin coupled to phycoerythrin. GeneChips were scanned using GeneChip Scanner 3000/7G and GeneChip Operating System (GCOS, Affymetrix, Santa Clara, USA) using Affymetrix default settings. 


\section{Microarray analysis}

Images of the Human Genome U133 Plus 2.0 arrays were quantified with GCOS software (Affymetrix). The chip description file (CDF) used for the analysis was an update created and freely distributed by the microarray lab of the university of Michigan (http://brainarray.mbni.med.umich.edu) ${ }^{36}$ based on UniGenes (version 8). A more detailed description of this analysis is shown in the supplementary data (Statistics S1). Briefly, the genes were analysed using a multivariate Gaussian linear regression including the hybridisation and labeling spikes, the hybridsation day, and a random effect to take into account multiple observations on the same subject. The inference criterion used for comparing the models is their ability to predict the observed data, i.e. models are compared directly through their minimised minus loglikelihood. When the numbers of parameters in models differ, they are penalised by adding the number of estimated parameters, a form of the Akaike Information Criterion $(\mathrm{AIC})^{37}$. For each gene, the treatment group was then added to the model. The gene under consideration was found to be differentially expressed if the AIC decreased compared to the model not containing the treatment effect. Effects are considered significant if the $95 \%$ confidence intervals do not overlap. This analysis method avoids multiple testing issues and improves statistical power compared to the conventional approach.

\section{Pathway analysis}

The genes analysed and fold changes were loaded into GenMapp (http://genmapp.org) ${ }^{38}$ and MAPPFinder ${ }^{39}$ software packages to evaluate the transcripts in relation to known biological processes, molecular function and cellular component based on Gene Ontology (GO) terms ${ }^{40}$ and contributed maps (i.e. local MAPPs). Only gene-transcripts with either their average intensities for the control and treated groups above 250 or average intensities for one of these groups above 500 and a $10 \%$ up or down regulation fold change were used to obtain a ranked list of pathways with differentially expressed genes.

MappFinder software was used to select the MAPPs with relatively high numbers of differentially expressed genes. The ranking of regulated pathways was indicated by the individual Z-scores. The Z-score increases when higher numbers of changing gene reporters relative to the number of genes on the MAPP are found on MAPPs. All pathways with both the Z-score and the number of genes changed $>1$ were considered to be significantly regulated. The results of the pathway analysis are presented in GO annotations (Table S3.1) and local MAPPs (Table S3.2), which give a more precise representation of the biological pathways in which the measured genes are involved.

Transcriptional data are published in the public database "ArrayExpress" ${ }^{41}$ under accession number E-MEXP-1705. 


\section{Real-Time PCR}

First strand cDNA was synthesised using the iScript cDNA Synthese kit (Bio-Rad, Veenendaal, The Netherlands) according to the manufacturer's instructions. $500 \mathrm{ng}$ of the total RNA used for the microarray analysis was used as a template for the cDNA reaction. The cDNA was diluted with RNase free $\mathrm{H}_{2} \mathrm{O}$ to a concentration of $0.32 \mathrm{ng} / \mu \mathrm{l}$. IQ Sybr Green Supermix (Bio-Rad, Veenendaal, The Netherlands) was used for the QPCR. Each Q-PCR reaction contained $12.5 \mu \mathrm{l}$ iQ Sybr Green Supermix, $1 \mu \mathrm{l}$ of $10 \mu \mathrm{M}$ gene-specific forward and reverse primers, $4 \mu$ diluted cDNA template and $6.5 \mu \mathrm{l}$ sterile water. CANX, 18SrRNA and GAPDH were included as Housekeeping genes. Primers that were used are presented in Table 3.1.

Q-PCR reactions were run on the My IQ Single Color Real Time PCR Detection System (Bio-Rad, Veenendaal, The Netherlands). After 3 minutes of incubation at $95^{\circ} \mathrm{C}$, an amplification cycle program of 40 cycles of 10 seconds at $95^{\circ} \mathrm{C}$ and 45 seconds at $60^{\circ} \mathrm{C}$, followed by a melting program was initiated.

Table3.1 Genes that were selected for q-PCR analysis with the primers that were used.

\begin{tabular}{lll}
\hline Gene: & Sequence ID & Primer (forward, reverse): \\
\hline 18SrRNA & M10098 & GTAACCCTTGAACCCATT, CCATCCAATCGGTAGTAGCG \\
GAPDH & NM_002046 & TGCACCACCAACTGCTTAGC, GGCATGGACTGTGGTCATGAG \\
CANX & NM_001024649 & CCACTGCTCCTCCTTCATCTCC, CGGTATCGTCTTTCTTGGCTTTGG \\
ACADM & NM_000016 & GCCAGCGATGTTCAGATACTAGAGG, CCTTTCCAGGGCATACTTGGTAGC \\
GPX1 & NM_000581 & CCGACCCCAAGCTCATCACC, GATGTCAGGCTCGATGTCAATGG \\
GPX3 & NM_002084 & ACATGCCTACAGGTATGCGTGATTG, TGGAGTGGAGAACTGGAGAGAAGG \\
GSR & NM_000637 & CAGGGACTTGGGTGTGATGAAATGC, GAGGTAGGGTGAATGGCGACTGTG \\
NDUFA3 & NM_004542 & GGAGACAAAGATGGCTGCGAGAG, GTCAGTGGAGGTGCTCACAGTTTC \\
NDUFV1 & NM_007103 & GCCATCGCCCGCCTCATTG, CCGTCACCCAGAGCACAAATCG \\
\hline
\end{tabular}

\section{Results}

\section{Micro-array analysis}

We used microarray analysis to assess the expression levels of all human genes in colonic biopsies after butyrate treatment compared to placebo. In total, 501 genes were found to be differentially expressed after the butyrate intervention compared to placebo (Table S2.3). From those genes, 473 were up regulated and 28 down regulated.

\section{Pathway analysis}

Pathway analysis with Genmapp software delivered a list of significantly regulated pathways, ranked by z-score (Table S3.1 and S3.2). The butyrate intervention mainly regulated the citric acid cycle (TCA-cycle) (Figure 3.2), fatty acid transport and 
oxidation, electron transport (Figure 3.2), TNF-alpha signaling and oxidative stress related pathways. In the TCA cycle pathway, citrate synthase and some genes involved in the formation of $\alpha$-ketogluterate out of isocitrate were upregulated. In the pathway of fatty acid metabolism, genes for transport and oxidation of medium and long chain fatty acids were expressed differentially. The pathway analyses also showed a number of differentially expressed genes in the electron transport chain (Figure 3.2). Most of these genes (9 out of 14) were present in Complex I and III of the electron transport chain. In the oxidative stress pathway, a number of genes involved in glutathione metabolism (GPX1, GPX3 and GSR) were differentially expressed.

\section{Validation of microarray analysis by q-PCR}

Based on microarray- and pathway analyses, 6 genes of interest were selected for confirmation by q-PCR. The increased expression of Acadm, Gpx3, Gpx1 and Ndufa3, identified by the micro array analysis, was confirmed by q-PCR (Table 3.2). The gene reporters Ndufv1 and Gsr were upregulated in the micro array, but downregulated in the q-PCR analysis.

Table 3.2 Fold changes of q-PCR and microarray analysis for 7 genes of interest.

\begin{tabular}{lcc}
\hline Gene & Micro-array $(\mathrm{fc})$ & $\mathrm{q}-\mathrm{PCR}(\mathrm{fc})$ \\
\hline Acadm & 1.12 & 1.11 \\
Gpx3 & 1.25 & 1.15 \\
Gpx1 & 1.13 & 1.07 \\
Ndufv1 & 1.20 & 0.91 \\
Ndufa3 & 1.13 & 1.13 \\
Gsr & 1.11 & 0.93 \\
\hline
\end{tabular}




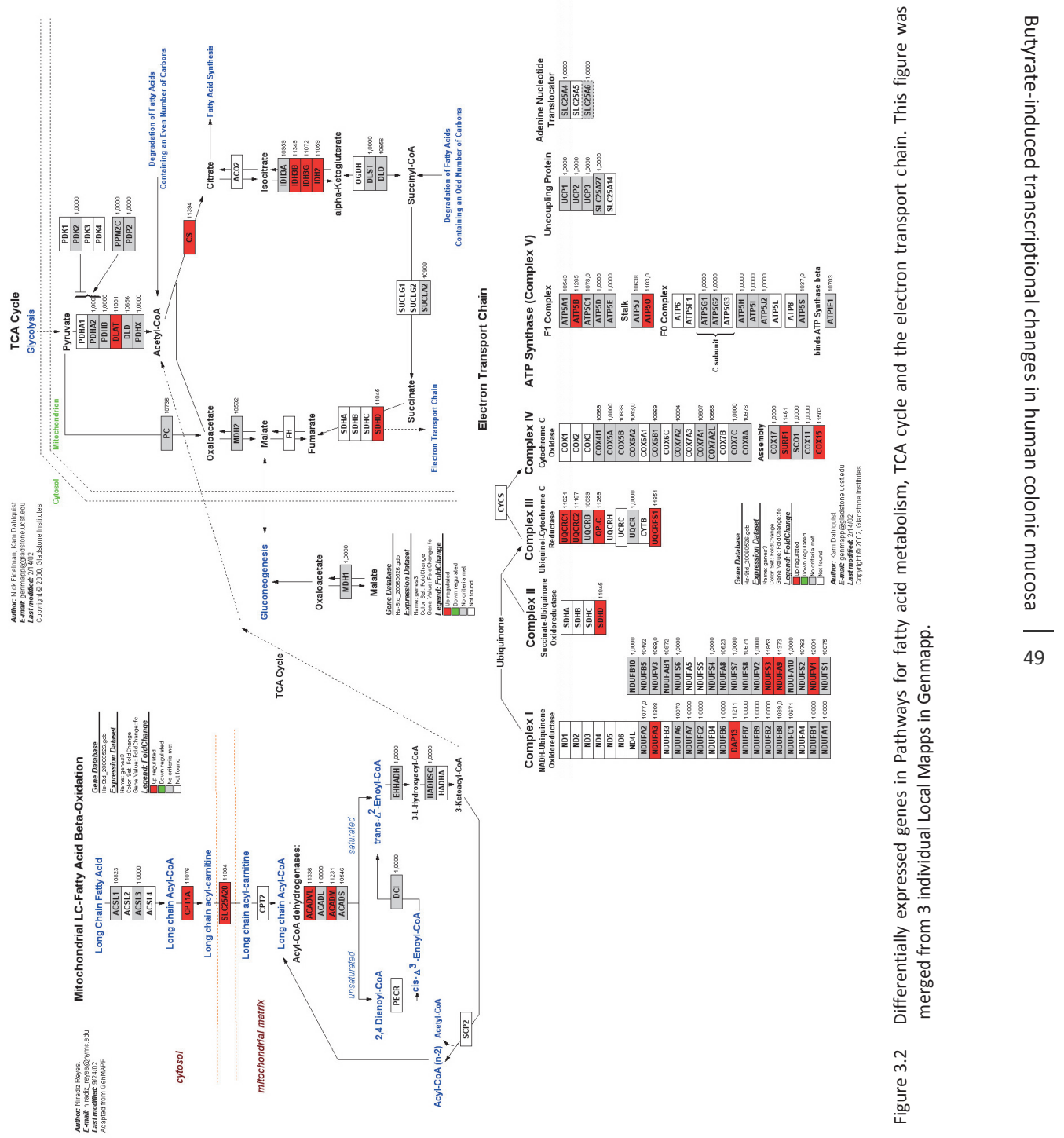




\section{Discussion}

Local administration of butyrate in the distal colon resulted in an increased transcription of genes, which were mainly associated with energy metabolism, fatty acid metabolism and oxidative stress. These results are in line with effects reported in literature, as reviewed recently by our group ${ }^{13}$. We showed for the first time that these processes are significantly regulated on the transcriptional level by intraluminal butyrate in healthy humans.

The impact of the butyrate administration as presented in this study with effects on gene transcription up to $39 \%$, was lower compared to previous findings in cell lines and animal studies, probably due to the fact that butyrate was studied in healthy volunteers in the most physiologically achievable way. Studying humans in vivo gives a larger variance in study data due to limitations of standardisation as well as the genomic variability compared to animal and in vitro studies. In contrast to stress models in animals and patients suffering from gastrointestinal disorders, healthy volunteers do not have a compromised gut. The beneficial effects that can be expected from the present intervention are, consequently, small compared to a compromised situation like in animals, cell lines or patients. The concentration of butyrate used in the present study (100 mM) was physiologically achievable by consuming a high fiber diet, in contrast to a pharmacological dose as used in some previous studies.

The microarray data show that fatty acid metabolism is regulated by butyrate, as a number of genes associated with processes involved in fatty acid transport, primary steps of beta oxidation and the formation of keton bodies were regulated. The transcription of genes encoding the fatty acid transporters carnitine palmityl-CoA transferase 1 (CPT1) and carnitine-acylcarnitine translocase (SLC25A20) was increased. CPT1 is located in the outer mitochondrial membrane and promotes the transport of long chain fatty acids into the mitochondrion by binding carnitine to the fatty acids ${ }^{42,43}$. Transport of carnitine-linked long chain fatty acids over the inner mitochondrial membrane is facilitated by SLC25A20 in exchange for free carnitine ${ }^{44,45}$. These two genes promote long chain fatty acid transport from the cytosol to the mitochondrial matrix where $\beta$-oxidation starts.

The first step of $\beta$-oxidation is the formation of 2-eonyl-CoA from the corresponding saturated ester, catalysed by SLC25A20. For dehydrogenation of acyl-CoA, 4 enzymes are described, each targeting fatty acids of a specific chain length:

short-chain-acyl-CoA dehydrogenase (ACADS, with C4 and C6 specificity), mediumchain-acyl-CoA dehydrogenase (ACADM, with C4-C12 specificity), long-chain-acyl-CoA dehydrogenase (ACADL, active with C8-C20) and very-long-chain-acyl-CoA dehydrogenase (ACADVL, active with $\mathrm{C} 12-\mathrm{C} 24)^{43}$. The butyrate (a C4 fatty acid) intervention resulted in an increased expression of both ACADM (confirmed with $q$ $P C R$ ), located in the mitochondrial matrix, and ACADVL, which is situated in the inner 
mitochondrial membrane. The intervention did not clearly modulate the transcriptional regulation of ACADS, in spite of its activity on C4-fatty acids. Next to mediating fatty acid transport, the rate of mitochondrial $\beta$-oxidation may also be limited by an accumulation of acetyl-CoA. This can be prevented by the observed increased transcription of both citrate synthase (CS), which drives the citric acid cycle, and by mitochondrial 3-hydroxy-3-methylglutaryl-CoA synthase (HMGCS2). HMGCS2 converts acetyl-CoA to ketone bodies ${ }^{46}$ thereby preventing the accumulation of acetyl-CoA $^{47}$. In humans, HMGCS2 is expressed in liver, skeletal muscle, heart, pancreas, testis and colon ${ }^{48}$. In rats, the expression of HMGCS2 in the colon depends on the amount of butyrate produced by the intestinal microbiota ${ }^{49,50}$. The mediation of fatty acid transport and HMGCS2 together with the increased ACADM and ACADVL expression suggests that butyrate is able to regulate the rate of fatty acid oxidation. Butyrate is known to inhibit proliferation in colonic tumor cells and cell lines ${ }^{7,12}$ but to stimulate proliferation in healthy colonic epithelial cells ${ }^{51,52}$. This is often referred to as "the butyrate paradox" inhibiting effect of butyrate on cell proliferation ${ }^{53}$. HMGCS2 expression in colonic epithelial cells is butyrate dependent and correlates with the capacity of the colon for ketogenesis and the fatty acid oxidation rate ${ }^{49,50}$. One explanation for the butyrate paradox is that healthy cells have an efficient butyrate metabolism resulting in low intracellular butyrate concentrations and therefore a decrease in capacity to inhibit growth $^{53}$. In colon cancer cell lines, $\beta$-oxidation and HMGCS2 expression are impaired $^{53}$. The decreased oxidation rate of butyrate may result in increased intracellular butyrate concentrations in tumor cells, hence causing increased histone deacetylation and subsequently decreased proliferation. The observation that butyrate affects proliferation is strengthened by the finding in the present study that several genes which are known to be involved in either proliferation, cell growth or cell size were differentially expressed by the butyrate intervention.

Butyrate mediated the transcription of genes that are involved in pyruvate dehydrogenase, citric acid cycle and the respiratory chain. The gene dihydrolipoamide acetyltransferase (DLAT), which encodes for a subunit of the pyruvate dehydrogenase complex forming acetyl-CoA from pyruvate was upregulated. Butyrate also increased the transcription of the genes that encode citrate synthase (CS) and succinate dehydrogenase (SDHD). Citrate synthase is the first enzyme of the TCA- cycle and catalyses the condensation of oxaloacetate, a cyclic acid cycle intermediate, and acetyl-CoA to form citrate. SDHD, which is also directly coupled to complex two of the electron transport chain, oxidises succinate to fumarate as the first part of the final step of the citric acid cycle ${ }^{54}$. Nicotinamide adenine dinucleotide (NADH) and flavin adenine dinucleotide $\left(\mathrm{FADH}_{2}\right)$, formed by glycolysis, the TCA-cycle and $\beta$-oxidation, subsequently enter the electron transport chain where the electrons are transferred along the respiratory chain in order to form ATP. Butyrate induced increased transcription of genes participating in all five complexes of the respiratory chain. In complex one, the genes NDUFA3 and NDUFV1 were significantly upregulated (and 
confirmed by q-PCR). SDHD, active in complex two of the respiratory chain, was upregulated. In total, 14 out of 72 genes that participate in the respiratory chain were upregulated (Table S3.2). In conclusion, butyrate regulated numerous genes involved in all parts of the total energy metabolism and ATP production, which provides molecular support for the general assumption that butyrate is involved in the energy metabolism of colonic epithelial cells.

Oxidative stress is an imbalance between the anti-oxidant defense network and the production of reactive oxygen species (ROS) and reactive nitrogen species (RNS). Butyrate has previously been shown to affect oxidative stress and inflammation ${ }^{13,55}$. In the present study, oxidative stress related pathways and NF- $\kappa B$ signaling were shown to be affected by butyrate. More specifically, the expression of glutathione peroxidases GPX 1 and GPX 3 and glutathione reductase (GSR) were upregulated in the oxidative stress pathway.

Glutathione (GSH) is used to eliminate reactive oxygen species, a reaction that is catalysed by glutathione peroxidase. The glutathione disulfide (GSSG) produced in this reaction can be converted back to GSH by the action of the enzyme glutathione reductase $(G S R)^{56}$. In the present study, the increased expression of GPX1, GPX3 and GSR suggests that butyrate induces an increased glutathione turnover capacity and an increased antioxidant capacity. This was in line with earlier findings from our group showing an increased GSH production after butyrate administration ${ }^{55}$. GSH can also detoxify harmful electrophils, and is catalysed by glutathione-S-transferase (GST). Previous studies showed that butyrate induced the expression levels of $\mathrm{GST}^{57,58}$. However, we did not observe transcriptional regulation of GST in the present study. The previously reported finding that butyrate mediates the JAK/STAT signaling pathway ${ }^{59}$, which plays an important role in the regulation of NO production in epithelial cells, supports the upregulation of one of the other genes in the oxidative stress pathway in the present study, NADH quinone oxidoreductase 1(NQO1), which also mediates nitric oxide (NO) biosynthesis. The current observation suggests that butyrate may increase stimulation of epithelial proliferation, migration and apoptosis. In an inflamed colon, reactive oxygen species (ROS) are produced by neutrophilic granulocytes, which are associated with increased oxidative stress as was previously reported in ulcerative colitis and Crohn's disease ${ }^{60,61}$. In the present study, the gene encoding nuclear factor kappa beta inhibitor $\alpha$ (NFKBIA), was upregulated. NFKBIA inhibits the activation of NF-KB and the TNF- $\alpha$ signaling cascade, thereby potentially leading to diminished inflammation and inflammation-induced oxidative stress.

Another important biological process affected by butyrate is proteasome degradation. This process, in which eight genes were differentially expressed (Table S3.2), provides a mechanism for degradation of (oxidatively) damaged proteins and the genes in this pathway are associated with apoptosis, ageing and oxidative stress ${ }^{62}$. 
Hence, butyrate regulated genes that are associated with glutathione metabolism, inflammation, NO synthesis and proteasome degradation, all supporting its previously demonstrated potential to reduce inflammation and oxidative stress.

The q-PCR analyses of 4 out of 6 genes confirmed the microarray results, whereas two genes were upregulated in the microarray analysis while they were downregulated in the q-PCR analysis. One gene was not significantly regulated based on the microarray results but showed a downregulation in the q-PCR analysis. There is international consensus that the Affymetrix microarray technology provides a reliable platform to measure gene expression ${ }^{63}$. The observed differences between microarray and q-PCR analysis are in line with earlier observations and can also be explained by differences in probe sequence and thus target location ${ }^{63}$. Because of these known problems to confirm microarray data with q-PCR, the relevance and importance of such a comparison should be reconsidered. Pathway analysis in combination with stringent statistical methods provides a strong indication for the quality of the microarray measurement. If pathway analysis results in significantly regulated pathways, a number of genes cluster within the same process, hence increasing the likelihood that the fold changes of these individual genes, measured simultaneously, were correct. The added value of the combination of both microarray analysis and the pathway analysis should be point of discussion when evaluating the reliability of the outcome of the microarray analysis.

Butyrate was administered by rectal enemas, because this is a safe and reliable way to deliver a specific amount of substrate to the distal colon. Other techniques, such as oral intake of dietary fibers or encapsulated butyrate, do not allow to accurately target the distal colon in vivo. The distal colon was chosen as target area for the butyrate intervention since the concentration of butyrate is lowest in this part of the colon due to rapid fermentation of commonly ingested dietary fibers in the proximal colon and the incidence of carcinomas and diseases in particularly the distal part of the colon is rising ${ }^{1,2}$. Furthermore, a mucosal specimen of the distal colon can be obtained much more easily compared to that of the proximal colon, without sedation and previous bowel cleansing. The latter is of pivotal importance in this type of studies to avoid disturbance of the physiological conditions in the gut, leading to interference with the effects of the intervention. Volunteers instilled the enemas in the evening prior to sleeping in order to obtain an optimal spread of the butyrate in the distal colon and to minimise the risk of leakage. The spread of enemas was studied previously in patients with ulcerative colitis ${ }^{64-67}$. In a pilot study in healthy volunteers, we confirmed, using enemas containing radio actively labeled Indium, that the spread of the $60 \mathrm{ml}$ enemas used in the present study reached beyond the sigmoid colon [unpublished data]. More research is needed on the actual concentration of butyrate at different locations in the human colon in vivo to optimise the dose and method of administration. 
This study showed for the first time in healthy volunteers the effect of butyrate treatment on the gene transcriptional level in the distal colon. Previously observed beneficial effects of butyrate from patient, animal- and in vitro studies are also induced in healthy subjects. The results presented in this study provide new leads to study the mechanisms involved in the effects of butyrate in humans. Future studies should be planned in order to study and optimize the functional consequences of butyrate or dietary fiber in the colon 


\section{Supplementary tables}

Table S3.1 GO-annotations, ranked by Z-score.

\begin{tabular}{|c|c|c|c|c|c|}
\hline GOID & GO Name & $\begin{array}{l}\text { Number } \\
\text { changed }\end{array}$ & $\begin{array}{l}\text { Number } \\
\text { measured }\end{array}$ & $\begin{array}{l}\text { Number } \\
\text { in GO }\end{array}$ & Z Score \\
\hline 8180 & signalosome complex & 2 & 2 & 9 & 9,299 \\
\hline 4128 & cytochrome-b5 reductase activity & 2 & 2 & 5 & 9,299 \\
\hline 5833 & hemoglobin complex & 2 & 3 & 9 & 7,505 \\
\hline 3756 & protein disulfide isomerase activity & 2 & 3 & 9 & 7,505 \\
\hline 5737 & cytoplasm & 58 & 1123 & 3067 & 7,269 \\
\hline 30126 & COPI vesicle coat & 2 & 4 & 8 & 6,424 \\
\hline 6672 & ceramide metabolism & 3 & 9 & 16 & 6,275 \\
\hline 3735 & structural constituent of ribosome & 6 & 33 & 309 & 6,169 \\
\hline 5739 & mitochondrion & 15 & 176 & 598 & 5,672 \\
\hline 5344 & oxygen transporter activity & 2 & 6 & 16 & 5,122 \\
\hline 6809 & nitric oxide biosynthesis & 2 & 6 & 15 & 5,122 \\
\hline 6635 & fatty acid beta-oxidation & 2 & 6 & 13 & 5,122 \\
\hline 6890 & retrograde vesicle-mediated transport \, Golgi to ER & 2 & 7 & 13 & 4,685 \\
\hline 15671 & oxygen transport & 2 & 7 & 17 & 4,685 \\
\hline 5840 & ribosome & 4 & 26 & 283 & 4,511 \\
\hline 8430 & selenium binding & 2 & 8 & 29 & 4,329 \\
\hline 6891 & intra-Golgi vesicle-mediated transport & 2 & 8 & 21 & 4,329 \\
\hline 8601 & protein phosphatase type $2 \mathrm{~A}$ regulator activity & 2 & 9 & 17 & 4,031 \\
\hline 9306 & protein secretion & 2 & 10 & 23 & 3,776 \\
\hline 6909 & phagocytosis & 2 & 10 & 22 & 3,776 \\
\hline 5622 & intracellular & 84 & 2775 & 7205 & 3,721 \\
\hline 5783 & endoplasmic reticulum & 11 & 172 & 474 & 3,701 \\
\hline 6118 & electron transport & 9 & 127 & 366 & 3,697 \\
\hline 16192 & vesicle-mediated transport & 10 & 150 & 335 & 3,676 \\
\hline 3824 & catalytic activity & 63 & 1916 & 4973 & 3,675 \\
\hline 5768 & endosome & 3 & 22 & 60 & 3,595 \\
\hline 16853 & isomerase activity & 4 & 36 & 135 & 3,582 \\
\hline 19825 & oxygen binding & 2 & 11 & 35 & 3,555 \\
\hline 6629 & lipid metabolism & 13 & 230 & 570 & 3,528 \\
\hline 8152 & metabolism & 82 & 2799 & 7383 & 3,274 \\
\hline 6457 & protein folding & 6 & 80 & 228 & 3,173 \\
\hline 6412 & protein biosynthesis & 10 & 185 & 644 & 2,922 \\
\hline 16491 & oxidoreductase activity & 11 & 213 & 655 & 2,902 \\
\hline 8021 & synaptic vesicle & 2 & 15 & 46 & 2,888 \\
\hline 6810 & transport & 34 & 974 & 2446 & 2,823 \\
\hline 30125 & clathrin vesicle coat & 2 & 16 & 36 & 2,758 \\
\hline 16740 & transferase activity & 24 & 639 & 1575 & 2,69 \\
\hline 6888 & ER to Golgi vesicle-mediated transport & 2 & 18 & 62 & 2,529 \\
\hline 8757 & S-adenosylmethionine-dependent methyltransferase activity & 3 & 35 & 85 & 2,518 \\
\hline 8415 & acyltransferase activity & 4 & 56 & 122 & 2,469 \\
\hline 6631 & fatty acid metabolism & 4 & 57 & 139 & 2,427 \\
\hline 51082 & unfolded protein binding & 4 & 66 & 159 & 2,088 \\
\hline 5975 & carbohydrate metabolism & 8 & 184 & 451 & 1,934 \\
\hline 6886 & intracellular protein transport & 6 & 128 & 365 & 1,867 \\
\hline 8380 & RNA splicing & 3 & 48 & 156 & 1,866 \\
\hline 1558 & regulation of cell growth & 3 & 49 & 108 & 1,826 \\
\hline 6310 & DNA recombination & 2 & 28 & 99 & 1,742 \\
\hline 5215 & transporter activity & 17 & 520 & 1265 & 1,619 \\
\hline
\end{tabular}




\begin{tabular}{llllll}
\hline GOID & GO Name & $\begin{array}{l}\text { Number } \\
\text { changed }\end{array}$ & $\begin{array}{l}\text { Number } \\
\text { measured }\end{array}$ & $\begin{array}{l}\text { Number } \\
\text { in GO }\end{array}$ & Z Score \\
\hline 20037 & heme binding & 2 & 31 & 87 & 1,573 \\
5625 & soluble fraction & 4 & 85 & 197 & 1,527 \\
8285 & negative regulation of cell proliferation & 3 & 58 & 140 & 1,499 \\
6470 & protein amino acid dephosphorylation & 3 & 59 & 131 & 1,466 \\
16829 & lyase activity & 3 & 60 & 149 & 1,434 \\
15031 & protein transport & 7 & 193 & 541 & 1,297 \\
5489 & electron transporter activity & 3 & 66 & 180 & 1,255 \\
139 & Golgi membrane & 2 & 38 & 82 & 1,249 \\
16757 & transferase activity transferring glycosyl groups & 4 & 97 & 224 & 1,244 \\
3714 & transcription corepressor activity & 2 & 39 & 88 & 1,208 \\
4295 & trypsin activity & 2 & 39 & 111 & 1,208 \\
6508 & proteolysis & 8 & 245 & 608 & 1,08 \\
74 & regulation of progression through cell cycle & 6 & 175 & 424 & 1,054 \\
9117 & nucleotide metabolism & 3 & 74 & 197 & 1,044 \\
8283 & cell proliferation & 7 & 213 & 503 & 1,025 \\
4 & biological process unknown & 8 & 250 & 705 & 1,02 \\
8372 & cellular component unknown & 9 & 288 & 768 & 1,011 \\
\hline
\end{tabular}

Table S3.2 Local mapps, ranked by Z-score.

\begin{tabular}{|c|c|c|c|c|}
\hline MAPP Name & $\begin{array}{l}\text { Number } \\
\text { changed }\end{array}$ & $\begin{array}{l}\text { Number } \\
\text { measured }\end{array}$ & $\begin{array}{l}\text { Number on } \\
\text { MAPP }\end{array}$ & Z Score \\
\hline Hs_Electron_Transport_Chain & 14 & 72 & 105 & 6,46 \\
\hline Hs_Krebs-TCA_Cycle & 6 & 19 & 31 & 5,92 \\
\hline Hs_Proteasome_Degradation & 8 & 36 & 61 & 5,38 \\
\hline Hs_Mitochondrial_fatty_acid_betaoxidation & 4 & 11 & 16 & 5,29 \\
\hline Hs_Oxidative_Stress & 6 & 24 & 28 & 5,06 \\
\hline Hs_Fatty_Acid_Beta_Oxidation_Meta_BiGCaT & 5 & 20 & 32 & 4,62 \\
\hline Hs_Glyoxylate_and_dicarboxylate_metabolism & 2 & 5 & 59 & 3,97 \\
\hline Hs_Aminosugars_metabolism & 3 & 14 & 54 & 3,19 \\
\hline Hs_Fatty_Acid_Beta_Oxidation_1_BiGCaT & 3 & 15 & 27 & 3,03 \\
\hline Hs_Valine_leucine_and_isoleucine_degradation & 3 & 16 & 54 & 2,88 \\
\hline Hs_Pyruvate_metabolism & 3 & 17 & 84 & 2,75 \\
\hline Hs_Glycogen_Metabolism & 4 & 30 & 36 & 2,48 \\
\hline Hs_Selenoamino_acid_metabolism & 2 & 11 & 39 & 2,30 \\
\hline Hs_Nucleotide_Metabolism & 2 & 12 & 17 & 2,14 \\
\hline Hs_Ribosomal_Proteins & 4 & 42 & 88 & 1,71 \\
\hline Hs_G13_Signaling_Pathway & 3 & 28 & 37 & 1,70 \\
\hline Hs_Fatty_acid_metabolism & 3 & 28 & 80 & 1,70 \\
\hline Hs_IL-1_NetPath_13 & 3 & 29 & 38 & 1,64 \\
\hline Hs_S1P_Signaling & 2 & 17 & 25 & 1,54 \\
\hline Hs_B_Cell_Receptor_NetPath_12 & 8 & 113 & 158 & 1,52 \\
\hline Hs_Glycolysis_and_Gluconeogenesis & 3 & 32 & 44 & 1,45 \\
\hline Hs_Butanoate_metabolism & 2 & 20 & 75 & 1,28 \\
\hline Hs_Lysine_degradation & 2 & 21 & 75 & 1,20 \\
\hline Hs_Purine_metabolism & 4 & 54 & 181 & 1,16 \\
\hline Hs_IL-3_NetPath_15 & 5 & 74 & 101 & 1,08 \\
\hline
\end{tabular}


Table S3.3

\begin{tabular}{|c|c|c|c|c|c|c|c|c|c|}
\hline Gene & fc & gene & $\mathrm{fc}$ & gene & fc & gene & fc & gene & fc \\
\hline Hs.610283 & 0,733 & Hs.538286 & 1,102 & Hs. 271264 & 1,105 & Hs.533282 & 1,108 & Hs.579928 & 1,114 \\
\hline Hs.547580 & 0,774 & Hs.471975 & 1,102 & Hs.221889 & 1,105 & Hs.591923 & 1,108 & Hs.433951 & 1,114 \\
\hline Hs.626427 & 0,794 & Hs.515258 & 1,102 & Hs.299208 & 1,105 & Hs.593308 & 1,108 & Hs.160958 & 1,115 \\
\hline Hs.594654 & 0,799 & Hs.614801 & 1,102 & Hs.179565 & 1,105 & Hs. 82432 & 1,109 & Hs.622151 & 1,115 \\
\hline Hs.598331 & 0,811 & Hs.632768 & 1,102 & Hs. 21701 & 1,105 & Hs.491682 & 1,109 & Hs. 271510 & 1,115 \\
\hline Hs.623586 & 0,824 & Hs.17250 & 1,102 & Hs.50334 & 1,106 & Hs.515371 & 1,109 & Hs.496984 & 1,115 \\
\hline Hs.643116 & 0,836 & Hs.23862 & 1,102 & Hs.567571 & 1,106 & Hs.227729 & 1,109 & Hs.82609 & 1,115 \\
\hline Hs.8102 & 0,843 & Hs.19673 & 1,102 & Hs.403171 & 1,106 & Hs.118110 & 1,109 & Hs.376722 & 1,115 \\
\hline Hs.634850 & 0,844 & Hs.119251 & 1,102 & Hs.17949 & 1,106 & Hs.126221 & 1,109 & Hs.388004 & 1,115 \\
\hline Hs.619120 & 0,847 & Hs.472558 & 1,102 & Hs.211594 & 1,106 & Hs.568881 & 1,109 & Hs.505077 & 1,115 \\
\hline Hs.332156 & 0,850 & Hs.5741 & 1,102 & Hs.492407 & 1,106 & Hs.632415 & 1,109 & Hs.302287 & 1,115 \\
\hline Hs.593614 & 0,854 & Hs.594563 & 1,102 & Hs.513141 & 1,106 & Hs.284286 & 1,109 & Hs.511801 & 1,116 \\
\hline Hs.596164 & 0,855 & Hs.71787 & 1,102 & Hs.596458 & 1,106 & Hs. 250905 & 1,109 & Hs. 513230 & 1,116 \\
\hline Hs.624050 & 0,872 & Hs.155742 & 1,102 & Hs.406840 & 1,106 & Hs.406515 & 1,109 & Hs.518236 & 1,116 \\
\hline Hs.642601 & 0,876 & Hs.23198 & 1,102 & Hs.436187 & 1,106 & Hs.82002 & 1,109 & Hs.379754 & 1,116 \\
\hline Hs.596854 & 0,879 & Hs.516111 & 1,102 & Hs.75160 & 1,106 & Hs.278569 & 1,109 & Hs.523302 & 1,116 \\
\hline Hs.568045 & 0,882 & Hs.188606 & 1,103 & Hs.129719 & 1,106 & Hs.356766 & 1,109 & Hs.591333 & 1,116 \\
\hline Hs.592712 & 0,883 & Hs.288382 & 1,103 & Hs. 534575 & 1,106 & Hs.468864 & 1,109 & Hs.317192 & 1,116 \\
\hline Hs.150793 & 0,886 & Hs.558009 & 1,103 & Hs.119591 & 1,106 & Hs.212102 & 1,110 & Hs. 584846 & 1,116 \\
\hline Hs.631536 & 0,898 & Hs.531624 & 1,103 & Hs.627196 & 1,106 & Hs.5920 & 1,110 & Hs.610390 & 1,116 \\
\hline Hs.611638 & 0,900 & Hs.55235 & 1,103 & Hs.140309 & 1,106 & Hs.614308 & 1,110 & Hs.131188 & 1,117 \\
\hline Hs.636080 & 0,903 & Hs.44017 & 1,103 & Hs.155097 & 1,106 & Hs.596717 & 1,110 & Hs.471205 & 1,117 \\
\hline Hs.640380 & 0,906 & Hs.610436 & 1,103 & Hs. 271014 & 1,106 & Hs.632430 & 1,110 & Hs.202 & 1,117 \\
\hline Hs.593826 & 0,907 & Hs.9194 & 1,103 & Hs.595678 & 1,106 & Hs.77558 & 1,110 & Hs.515242 & 1,117 \\
\hline Hs.597007 & 0,907 & Hs.472793 & 1,103 & Hs. 25313 & 1,107 & Hs.130774 & 1,110 & Hs.621002 & 1,117 \\
\hline Hs.213087 & 0,909 & Hs.75659 & 1,103 & Hs.50130 & 1,107 & Hs.503345 & 1,110 & Hs.445899 & 1,118 \\
\hline Hs.630427 & 0,909 & Hs.104203 & 1,103 & Hs.5662 & 1,107 & Hs.58351 & 1,110 & Hs.515714 & 1,118 \\
\hline Hs.102798 & 1,100 & Hs.409140 & 1,103 & Hs.595426 & 1,107 & Hs.432330 & 1,110 & Hs.93659 & 1,118 \\
\hline Hs.16606 & 1,100 & Hs.497353 & 1,103 & Hs.24379 & 1,107 & Hs.14317 & 1,110 & Hs.324844 & 1,118 \\
\hline Hs.335551 & 1,100 & Hs.503911 & 1,103 & Hs.511251 & 1,107 & Hs.591998 & 1,110 & Hs.636012 & 1,118 \\
\hline Hs.591145 & 1,100 & Hs. 467408 & 1,103 & Hs.514174 & 1,107 & Hs.592920 & 1,110 & Hs.587054 & 1,118 \\
\hline Hs.636329 & 1,100 & Hs.111903 & 1,103 & Hs.580808 & 1,107 & Hs. 567405 & 1,111 & Hs.400740 & 1,118 \\
\hline Hs.298716 & 1,100 & Hs. 443723 & 1,103 & Hs.595864 & 1,107 & Hs.592781 & 1,111 & Hs.133968 & 1,118 \\
\hline Hs.437779 & 1,100 & Hs.32018 & 1,104 & Hs. 268530 & 1,107 & Hs.414809 & 1,111 & Hs. 280378 & 1,118 \\
\hline Hs.532699 & 1,100 & Hs.599024 & 1,104 & Hs.328865 & 1,107 & Hs.418450 & 1,111 & Hs.504620 & 1,119 \\
\hline Hs.555926 & 1,100 & Hs.76244 & 1,104 & Hs.452445 & 1,107 & Hs.504609 & 1,111 & Hs.306764 & 1,119 \\
\hline Hs.514199 & 1,101 & Hs.502004 & 1,104 & Hs.284141 & 1,107 & Hs.604630 & 1,111 & Hs.368610 & 1,119 \\
\hline Hs. 258429 & 1,101 & Hs.26216 & 1,104 & Hs.466165 & 1,107 & Hs.430733 & 1,111 & Hs.405061 & 1,119 \\
\hline Hs.292009 & 1,101 & Hs.483454 & 1,104 & Hs.501280 & 1,107 & Hs.63290 & 1,111 & Hs.592048 & 1,119 \\
\hline Hs.502914 & 1,101 & Hs. 514373 & 1,104 & Hs.130413 & 1,107 & Hs. 232543 & 1,111 & Hs.321689 & 1,119 \\
\hline Hs.511138 & 1,101 & Hs.1376 & 1,104 & Hs.154073 & 1,107 & Hs.597689 & 1,111 & Hs.404119 & 1,119 \\
\hline Hs. 567523 & 1,101 & Hs.4993 & 1,104 & Hs.410197 & 1,107 & Hs.445570 & 1,111 & Hs.447011 & 1,119 \\
\hline Hs.200738 & 1,101 & Hs.627962 & 1,104 & Hs.514065 & 1,107 & Hs.491988 & 1,111 & Hs.525527 & 1,119 \\
\hline Hs.632469 & 1,101 & Hs.72363 & 1,104 & Hs. 289271 & 1,107 & Hs.591729 & 1,111 & Hs. 242458 & 1,119 \\
\hline Hs. 77422 & 1,101 & Hs. 464422 & 1,104 & Hs. 624418 & 1,107 & Hs.619260 & 1,112 & Hs.333579 & 1,119 \\
\hline Hs.78888 & 1,101 & Hs. 505652 & 1,104 & Hs.140452 & 1,108 & Hs.95577 & 1,112 & Hs.596307 & 1,119 \\
\hline Hs. 213534 & 1,101 & Hs.38972 & 1,104 & Hs.268849 & 1,108 & Hs.513987 & 1,112 & Hs.284295 & 1,120 \\
\hline Hs.446017 & 1,101 & Hs.484188 & 1,104 & Hs.469171 & 1,108 & Hs.406068 & 1,112 & Hs.461379 & 1,120 \\
\hline Hs.567366 & 1,101 & Hs.632215 & 1,104 & Hs.503043 & 1,108 & Hs. 81328 & 1,112 & Hs. 272805 & 1,120 \\
\hline Hs.250758 & 1,101 & Hs.356769 & 1,104 & Hs.505676 & 1,108 & Hs. 256632 & 1,112 & Hs.355606 & 1,121 \\
\hline Hs. 421724 & 1,101 & Hs.595752 & 1,104 & Hs.592292 & 1,108 & Hs.592970 & 1,112 & Hs.9825 & 1,121 \\
\hline Hs.128676 & 1,101 & Hs.356270 & 1,105 & Hs.6076 & 1,108 & Hs.432792 & 1,113 & Hs.163543 & 1,121 \\
\hline Hs.591332 & 1,101 & Hs.529571 & 1,105 & Hs.25597 & 1,108 & Hs. 603284 & 1,113 & Hs.436121 & 1,121 \\
\hline Hs.593679 & 1,101 & Hs. 584950 & 1,105 & Hs.465645 & 1,108 & Hs.434401 & 1,113 & Hs.593910 & 1,121 \\
\hline Hs.595644 & 1,101 & Hs.280990 & 1,105 & Hs.598842 & 1,108 & Hs.401537 & 1,113 & Hs.437388 & 1,121 \\
\hline Hs.528763 & 1,101 & Hs. 567488 & 1,105 & Hs.482038 & 1,108 & Hs.408073 & 1,113 & Hs. 606526 & 1,121 \\
\hline Hs.6686 & 1,101 & Hs. 614545 & 1,105 & Hs.15591 & 1,108 & Hs. 241543 & 1,114 & Hs.9857 & 1,121 \\
\hline Hs.527412 & 1,102 & Hs.495985 & 1,105 & Hs.31431 & 1,108 & Hs.493164 & 1,114 & Hs.33642 & 1,121 \\
\hline
\end{tabular}




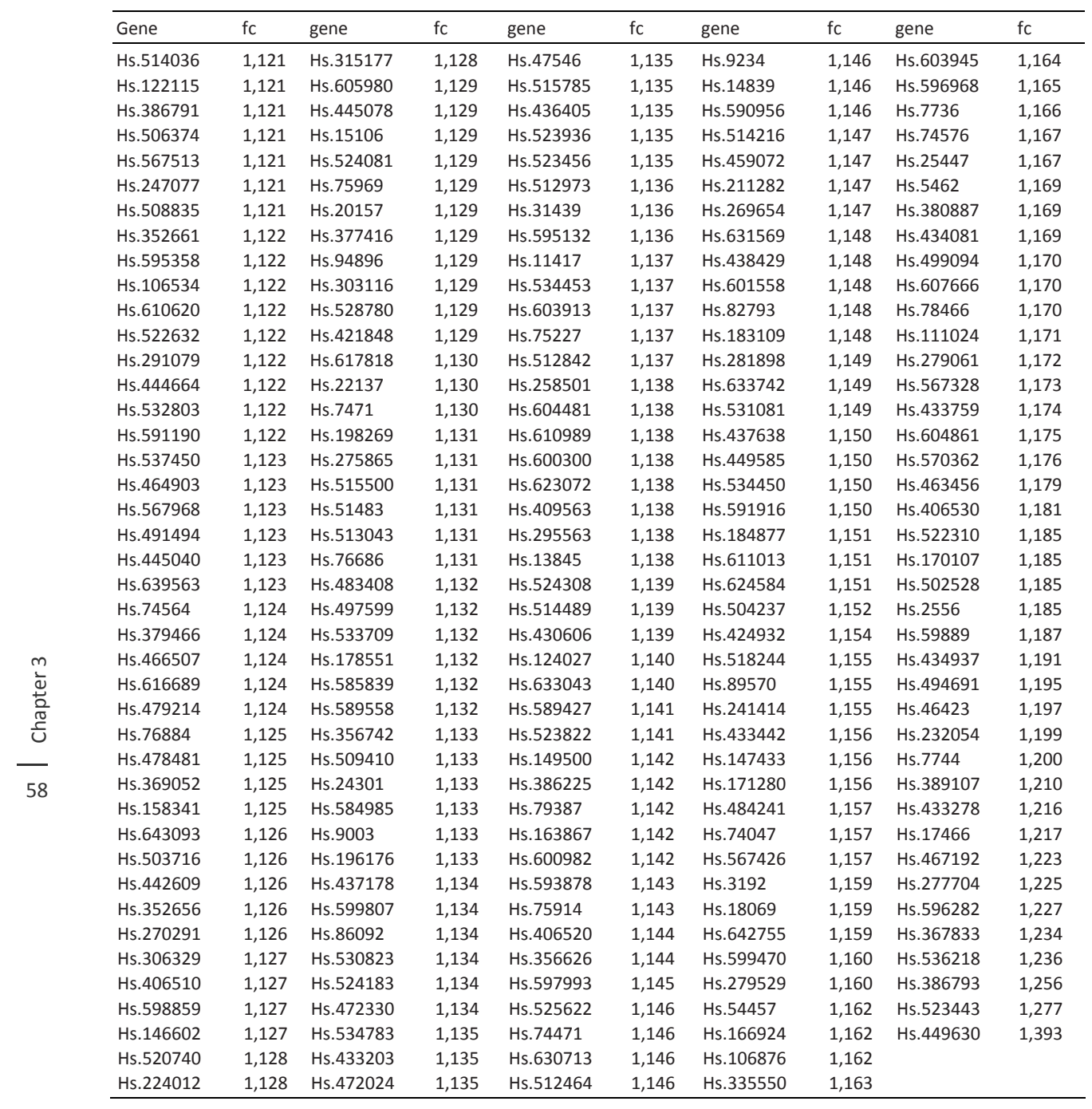




\section{References}

1. Marteau P. Probiotics, prebiotics, synbiotics: ecological treatment for inflammatory bowel disease? Gut 2006;55:1692-3.

2. Le Leu RK, Brown IL, Hu Y, Morita T, Esterman A, et al. Effect of dietary resistant starch and protein on colonic fermentation and intestinal tumourigenesis in rats. Carcinogenesis 2007;28:240-5.

3. Wong CS, Sengupta S, Tjandra JJ, Gibson PR. The influence of specific luminal factors on the colonic epithelium: high-dose butyrate and physical changes suppress early carcinogenic events in rats. Dis Colon Rectum 2005;48: 549-59.

4. Bergman EN. Energy contributions of volatile fatty acids from the gastrointestinal tract in various species. Physiol Rev 1990;70:567-90.

5. Topping DL, Clifton PM. Short-chain fatty acids and human colonic function: roles of resistant starch and nonstarch polysaccharides. Physiol Rev 2001;81:1031-64.

6. Hallert C, Bjorck I, Nyman M, Pousette A, Granno C, et al. Increasing fecal butyrate in ulcerative colitis patients by diet: controlled pilot study. Inflamm Bowel Dis 2003;9:116-21.

7. Comalada M, Bailon E, de Haro O, Lara-Villoslada F, Xaus J, et al. The effects of short-chain fatty acids on colon epithelial proliferation and survival depend on the cellular phenotype. J Cancer Res Clin Oncol 2006;132:487-97.

8. Ranganna K, Joshi T, Yatsu FM. Sodium butyrate inhibits platelet-derived growth factor-induced proliferation of vascular smooth muscle cells. Arterioscler Thromb Vasc Biol 1995;15:2273-83.

9. Ranganna K, Yatsu FM, Hayes BE, Milton SG, Jayakumar A. Butyrate inhibits proliferation-induced proliferating cell nuclear antigen expression (PCNA) in rat vascular smooth muscle cells. Mol Cell Biochem 2000;205:149-61.

10. Prasad KN. Butyric acid: a small fatty acid with diverse biological functions. Life Sci 1980;27:1351-8.

11. Hague A, Butt AJ, Paraskeva C. The role of butyrate in human colonic epithelial cells: an energy source or inducer of differentiation and apoptosis? Proc Nutr Soc 1996;55:937-43.

12. Hodin RA, Meng S, Archer S, Tang R. Cellular growth state differentially regulates enterocyte gene expression in butyrate-treated HT-29 cells. Cell Growth Differ 1996;7:647-53.

13. Hamer HM, Jonkers D, Venema K, Vanhoutvin S, Troost FJ, et al. Review article: the role of butyrate on colonic function. Aliment Pharmacol Ther 2008;27:104-19.

14. Cuff M, Dyer J, Jones M, Shirazi-Beechey S. The human colonic monocarboxylate transporter Isoform 1: its potential importance to colonic tissue homeostasis. Gastroenterology 2005;128:676-86.

15. Pajak B, Orzechowski A, Gajkowska B. Molecular basis of sodium butyrate-dependent proapoptotic activity in cancer cells. Adv Med Sci 2007;52:83-8.

16. Mariadason JM, Kilias D, Catto-Smith A, Gibson PR. Effect of butyrate on paracellular permeability in rat distal colonic mucosa ex vivo. J Gastroenterol Hepatol 1999;14:873-9.

17. Peng L, He Z, Chen W, Holzman IR, Lin J. Effects of butyrate on intestinal barrier function in a Caco-2 cell monolayer model of intestinal barrier. Pediatr Res 2007;61:37-41.

18. Vanhoutvin SA, Troost FJ, Kilkens TO, Lindsey PJ, Hamer HM, et al. The effects of butyrate enemas on visceral perception in healthy volunteers. Neurogastroenterol Motil. 2009;21:952-e76.

19. Cherbut C. Motor effects of short-chain fatty acids and lactate in the gastrointestinal tract. Proc Nutr Soc 2003;62:95-9.

20. Cani PD, Joly E, Horsmans $Y$, Delzenne NM. Oligofructose promotes satiety in healthy human: a pilot study. Eur J Clin Nutr 2006;60:567-72.

21. Daly K, Shirazi-Beechey SP. Microarray analysis of butyrate regulated genes in colonic epithelial cells. DNA Cell Biol 2006;25:49-62.

22. Li RW, Li C. Butyrate induces profound changes in gene expression related to multiple signal pathways in bovine kidney epithelial cells. BMC Genomics 2006;7:234.

23. Daly K, Cuff MA, Fung F, Shirazi-Beechey SP. The importance of colonic butyrate transport to the regulation of genes associated with colonic tissue homoeostasis. Biochem Soc Trans 2005;33: 733-5.

24. Li CJ, Li RW, Wang YH, Elsasser TH. Pathway analysis identifies perturbation of genetic networks induced by butyrate in a bovine kidney epithelial cell line. Funct Integr Genomics 2007;7:193-205.

25. Ranganna K, Yousefipour Z, Yatsu FM, Milton SG, Hayes BE. Gene expression profile of butyrateinhibited vascular smooth muscle cell proliferation. Mol Cell Biochem 2003;254:21-36. 
26. Tabuchi Y, Takasaki I, Doi T, Ishii Y, Sakai H, et al. Genetic networks responsive to sodium butyrate in colonic epithelial cells. FEBS Lett 2006;580:3035-41.

27. Joseph J, Mudduluru G, Antony S, Vashistha S, Ajitkumar P, et al. Expression profiling of sodium butyrate $(\mathrm{NaB})$-treated cells: identification of regulation of genes related to cytokine signaling and cancer metastasis by NaB. Oncogene 2004;23:6304-15.

28. Mariadason JM, Corner GA, Augenlicht LH. Genetic reprogramming in pathways of colonic cell maturation induced by short chain fatty acids: comparison with trichostatin A, sulindac, and curcumin and implications for chemoprevention of colon cancer. Cancer Res 2000;60:4561-72.

29. Miller SJ, Zaloga GP, Hoggatt AM, Labarrere C, Faulk WP. Short-chain fatty acids modulate gene expression for vascular endothelial cell adhesion molecules. Nutrition 2005;21:740-8.

30. Thibault R, De Coppet P, Daly K, Bourreille A, Cuff M, et al. Down-regulation of the monocarboxylate transporter 1 is involved in butyrate deficiency during intestinal inflammation. Gastroenterology 2007;133:1916-27.

31. Williams EA, Coxhead JM, Mathers JC. Anti-cancer effects of butyrate: use of micro-array technology to investigate mechanisms. Proc Nutr Soc 2003;62:107-15.

32. Bordin M, D'Atri F, Guillemot L, Citi S. Histone deacetylase inhibitors up-regulate the expression of tight junction proteins. Mol Cancer Res 2004;2:692-701.

33. Kien $\mathrm{CL}$, Blauwiekel $\mathrm{R}$, Bunn JY, Jetton TL, Frankel WL, et al. Cecal infusion of butyrate increases intestinal cell proliferation in piglets. J Nutr 2007;137:916-22.

34. Kien CL, Peltier CP, Mandal S, Davie JR, Blauwiekel R. Effects of the in vivo supply of butyrate on histone acetylation of cecum in piglets. JPEN J Parenter Enteral Nutr 2008;32:51-6.

35. Gaudier E, Rival M, Buisine M, Robineau I, Hoebler C. Butyrate enemas upregulate Muc genes expression but decrease adherent mucus thickness in mice colon. Physiol Res. 2009;58:111-9.

36. Dai M, Wang P, Boyd AD, Kostov G, Athey B, et al. Evolving gene/transcript definitions significantly alter the interpretation of GeneChip data. Nucleic Acids Res 2005;33:e175.

37. Akaike H. Information theory and an extension of the maximum likelihood principle. Second international symposium on inference theory (Tsahkadsor, 1971) 1973:267-81.

38. Dahlquist KD, Salomonis N, Vranizan K, Lawlor SC, Conklin BR. GenMAPP, a new tool for viewing and analyzing microarray data on biological pathways. Nat Genet 2002;31:19-20.

39. Doniger SW, Salomonis N, Dahlquist KD, Vranizan K, Lawlor SC, et al. MAPPFinder: using Gene Ontology and GenMAPP to create a global gene-expression profile from microarray data. Genome Biol 2003;4:R7.

40. Ashburner M, Ball CA, Blake JA, Botstein D, Butler H, et al. Gene ontology: tool for the unification of biology. The Gene Ontology Consortium. Nat Genet 2000;25:25-9.

41. Parkinson H, Kapushesky M, Kolesnikov N, Rustici G, Shojatalab M, et al. ArrayExpress update--from an archive of functional genomics experiments to the atlas of gene expression. Nucleic Acids Res 2009;37:D868-72.

42. Gregersen N, Andresen BS, Corydon MJ, Corydon TJ, Olsen RK, et al. Mutation analysis in mitochondrial fatty acid oxidation defects: Exemplified by acyl-CoA dehydrogenase deficiencies, with special focus on genotype-phenotype relationship. Hum Mutat 2001;18:169-89.

43. Eaton S, Bartlett K, Pourfarzam M. Mammalian mitochondrial beta-oxidation. Biochem J 1996;320 (Pt 2):345-57.

44. Korman SH, Pitt JJ, Boneh A, Dweikat I, Zater M, et al. A novel SLC25A20 splicing mutation in patients of different ethnic origin with neonatally lethal carnitine-acylcarnitine translocase (CACT) deficiency. Mol Genet Metab 2006;89:332-8.

45. Indiveri C, lacobazzi V, Giangregorio N, Palmieri F. The mitochondrial carnitine carrier protein: cDNA cloning, primary structure and comparison with other mitochondrial transport proteins. Biochem J 1997;321 ( Pt 3):713-9.

46. Dashti N, Ontko JA. Rate-limiting function of 3-hydroxy-3-methylglutaryl-coenzyme A synthase in ketogenesis. Biochem Med 1979;22:365-74.

47. Le May C, Pineau T, Bigot K, Kohl C, Girard J, et al. Reduced hepatic fatty acid oxidation in fasting PPARalpha null mice is due to impaired mitochondrial hydroxymethylglutaryl-CoA synthase gene expression. FEBS Lett 2000;475:163-6.

48. Mascaro C, Buesa C, Ortiz JA, Haro D, Hegardt FG. Molecular cloning and tissue expression of human mitochondrial 3-hydroxy-3-methylglutaryl-CoA synthase. Arch Biochem Biophys 1995;317:385-90. 
49. Cherbuy C, Darcy-Vrillon B, Morel MT, Pegorier JP, Duee PH. Effect of germfree state on the capacities of isolated rat colonocytes to metabolize n-butyrate, glucose, and glutamine. Gastroenterology 1995;109:1890-9.

50. Cherbuy C, Andrieux C, Honvo-Houeto E, Thomas M, Ide C, et al. Expression of mitochondrial HMGCoA synthase and glutaminase in the colonic mucosa is modulated by bacterial species. Eur J Biochem 2004;271:87-95.

51. Mortensen FV, Langkilde NC, Joergensen JC, Hessov I. Short-chain fatty acids stimulate mucosal cell proliferation in the closed human rectum after Hartmann's procedure. Int J Colorectal Dis 1999;14: 150-4.

52. Scheppach W, Bartram P, Richter A, Richter F, Liepold H, et al. Effect of short-chain fatty acids on the human colonic mucosa in vitro. JPEN J Parenter Enteral Nutr 1992;16:43-8.

53. Camarero N, Mascaro C, Mayordomo C, Vilardell F, Haro D, et al. Ketogenic HMGCS2 Is a c-Myc target gene expressed in differentiated cells of human colonic epithelium and down-regulated in colon cancer. Mol Cancer Res 2006;4:645-53.

54. Stryer L. Biochemistry: WH Freeman and Company New York 1997.

55. Hamer HM, Jonkers DM, Bast A, Vanhoutvin SA, Fischer MA, et al. Butyrate modulates oxidative stress in the colonic mucosa of healthy humans. Clin Nutr 2009;28:88-93.

56. Maher P. The effects of stress and aging on glutathione metabolism. Ageing Res Rev 2005;4: 288-314.

57. Pool-Zobel BL, Selvaraju V, Sauer J, Kautenburger T, Kiefer J, et al. Butyrate may enhance toxicological defence in primary, adenoma and tumor human colon cells by favourably modulating expression of glutathione S-transferases genes, an approach in nutrigenomics. Carcinogenesis 2005;26: 1064-76.

58. Ebert MN, Klinder A, Peters WH, Schaferhenrich A, Sendt W, et al. Expression of glutathione S-transferases (GSTs) in human colon cells and inducibility of GSTM2 by butyrate. Carcinogenesis 2003;24:1637-44.

59. Stempelj M, Kedinger M, Augenlicht L, Klampfer L. Essential role of the JAK/STAT1 signaling pathway in the expression of inducible nitric-oxide synthase in intestinal epithelial cells and its regulation by butyrate. J Biol Chem 2007;282:9797-804.

60. Kruidenier L, Verspaget HW. Review article: oxidative stress as a pathogenic factor in inflammatory bowel disease--radicals or ridiculous? Aliment Pharmacol Ther 2002;16:1997-2015.

61. Kruidenier L, Kuiper I, Lamers CB, Verspaget HW. Intestinal oxidative damage in inflammatory bowel disease: semi-quantification, localization, and association with mucosal antioxidants. J Pathol 2003;201: 28-36.

62. Glickman $\mathrm{MH}$, Ciechanover A. The ubiquitin-proteasome proteolytic pathway: destruction for the sake of construction. Physiol Rev 2002;82:373-428.

63. Canales RD, Luo Y, Willey JC, Austermiller B, Barbacioru CC, et al. Evaluation of DNA microarray results with quantitative gene expression platforms. Nat Biotechnol 2006;24:1115-22.

64. Campieri M, Corbelli C, Gionchetti P, Brignola C, Belluzzi A, et al. Spread and distribution of 5-ASA colonic foam and 5-ASA enema in patients with ulcerative colitis. Dig Dis Sci 1992;37:1890-7.

65. Chapman NJ, Brown ML, Phillips SF, Tremaine WJ, Schroeder KW, et al. Distribution of mesalamine enemas in patients with active distal ulcerative colitis. Mayo Clin Proc 1992;67:245-8.

66. Kruis W, Bull U, Eisenburg J, Paumgartner G. Retrograde colonic spread of sulphasalazine enemas. Scand J Gastroenterol 1982;17:933-8.

67. Vitti RA, Meyers F, Knight LC, Siegel JA, Malmud LS, et al. Quantitative distribution of radiolabeled 5-aminosalicylic acid enemas in patients with left-sided ulcerative colitis. Dig Dis Sci 1989;34: 1792-7. 


\section{4}

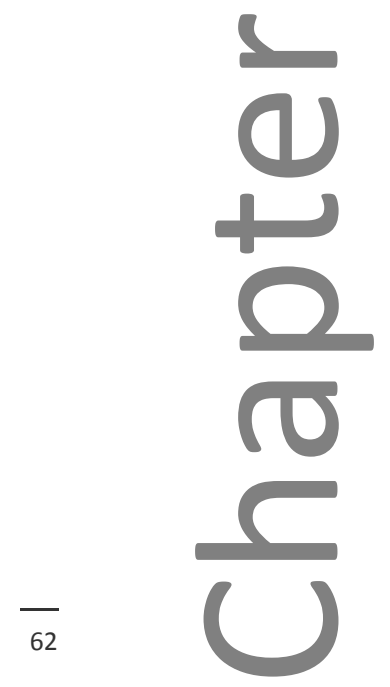




\section{The effects of butyrate enemas on visceral perception in healthy volunteers}

Steven ALW Vanhoutvin, Freddy J Troost, Tessa Kilkens,

Patrick .J Lindsey, Henrike M Hamer, Daisy MAE Jonkers, Koen Venema, Robert JM Brummer

Neurogastroenterol Motil. 2009;21:952-e76 


\section{Abstract}

\section{Introduction}

Fermentation of dietary fibers by colonic microbes leads to the production of short chain fatty acids (mainly propionate, butyrate and acetate), which are utilized by the colonic mucosa. Previous studies showed positive effects of butyrate on parameters of oxidative stress, inflammation and apoptosis. Recent studies in rats, however, showed that butyrate increased visceral sensitivity.

\section{Objectives}

The aim of this study was to determine the effects of physiologically relevant concentrations of butyrate on visceral perception in healthy human subjects.

\section{Methods}

Eleven healthy volunteers participated in this randomized double blind, placebo controlled cross over study. The study consisted of three periods of one week each, in which the volunteers daily self-administered rectal enemas containing $100 \mathrm{mM}, 50 \mathrm{mM}$ butyrate, or placebo (saline) prior to sleeping. A rectal barostat measurement was performed at the start and the end of each test period for the measurement of pain, urge and discomfort.

\section{Results}

Butyrate treatment resulted in a dose-dependent reduction of pain, urge and discomfort throughout the entire pressure range of the protocol. At a pressure of $4 \mathrm{mmHg}, 50 \mathrm{mM}$ and 100 $\mathrm{mM}$ butyrate concentrations resulted in a $23.9 \%$ and $42.1 \%$ reduction of pain scores, respectively, and the discomfort scores decreased by $44.2 \%$ and $69.0 \%$, respectively. At a pressure of $67 \mathrm{mmHg}, 50$ and $100 \mathrm{mM}$ of butyrate decreased the pain scores by $23,8 \%$ and $42 \%$, respectively, and discomfort scores $1,9 \%$ and $5,2 \%$, respectively.

\section{Conclusion}

Colonic administration of butyrate, at physiologically relevant concentrations, dosedependently decreases visceral sensitivity in healthy volunteers. 


\section{Introduction}

The human large intestine harbors a complex diversity of micro-organisms, which exert both positive and negative effects on gut physiology. Short chain fatty acids (SCFAs) are derived from microbial fermentation of undigested dietary fibers in the colon. Most saccharolytic fermentation occurs in the proximal colon whereas in the distal colon the depletion of fermentable carbohydrates leads to a switch towards proteolytic fermentation, which is less favourable due to the formation of toxic end products (such as ammonia, sulphur compounds, indoles and phenolic compounds). The amount of SCFAs (mainly acetate, propionate and butyrate) produced in the colon depends on the site of fermentation, the diet and the composition of the microbiota, and can provide up to $5-15 \%$ of the substrates for human energy production ${ }^{1}$.

Among the different SCFAs, butyrate is known to modulate numerous processes ${ }^{2}$. Increased colonic butyrate formation has often been proposed as one of the protective mechanisms of high fiber diets ${ }^{3-5}$. Butyrate is the major energy source for colonocytes $^{6}$ and may act as a signal metabolite affecting epithelial cell proliferation, apoptosis and differentiation ${ }^{7}$. There is evidence that butyrate beneficially affects several inflammatory parameters such as cytokines and myeloperoxidase activity, primarily via inhibition of nuclear factor kappa B (NF-KB) activation ${ }^{8}$. Furthermore, butyrate stimulates intestinal mucus production, thereby supporting the mucosal barrier function ${ }^{9,10}$, increases anti-oxidant capacity ${ }^{11,12}$, increases mucosal blood flow $^{13}$, and may decrease colonic epithelial permeability ${ }^{14,15}$.

Although there is ample evidence for these beneficial effects from in vitro and animal models, limited studies have confirmed these effects in vivo in humans.

Previously, positive effects of butyrate on inflammation in active distal ulcerative colitis have been reported ${ }^{2}$. Paradoxically, two studies in rats showed that butyrate instillation in the colon increased visceral sensitivity and maintained an increased visceral sensitivity after TNBS induced colitis ${ }^{16,17}$. It is not known whether a comparable effect of butyrate administration on visceral sensitivity can be observed in human subjects. The aim of this study therefore is to evaluate the effects of rectally administered butyrate, in a concentration that can be reached by consumption of a high fiber diet, on visceral perception in healthy volunteers.

\section{Materials and methods}

\section{Subjects}

Eleven healthy volunteers ( 3 male and 8 female) were included in this randomised, double blind, placebo-controlled cross-over study. None of the subjects had a history of gastrointestinal disease or previous abdominal surgery. The study was approved by 
the Medical Ethics Committee of University Hospital Maastricht and conducted in full accordance with the principles of the 'Declaration of Helsinki' (52nd WMA General Assembly, Edinburgh, Scotland, Oct 2000). All volunteers gave their written informed consent prior to participation. The study has been registered in the US National Library of Medicine (http://www.clinicaltrials.gov, NCT 00726817)

\section{Study design}

The study consisted of three periods of one week each, with a wash-out period of two weeks in between the test weeks. The volunteers self-administered rectal enemas (enema bottles were kindly provided by Tramedico Holding B.V., Weesp, The Netherlands) containing a $60 \mathrm{ml}$ solution of either butyrate (100 or $50 \mathrm{mM}$ ) or placebo (saline) once daily just prior to sleeping. During the first 20 minutes after enema instillation, volunteers were instructed to stay in a left lateral supine position. The enemas were made isotonic with sodium chloride and had a $\mathrm{pH}$ of 7 . Barostat measurements to assess visceral perception were performed at the start and at the end of each test week (Figure 4.1). Compliance of the enema administration and complaints were monitored using a daily questionnaire.

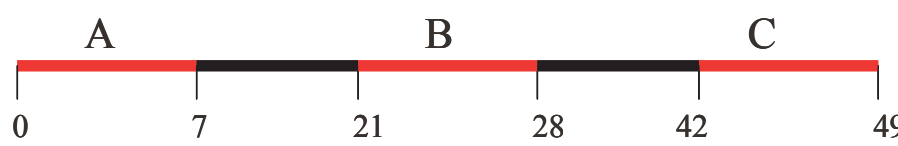
A: Placebo
B: Butyrate $100 \mathrm{mM}$
C: Butyrate $50 \mathrm{mM}$

Figure 4.1 Design of barostat study consisting of 3 randomised cross-over test periods of 7 days, interspaced with 14 days wash-out period.

\section{Barostat protocol}

After arrival in the hospital, the volunteers self-administered a rectal enema containing $60 \mathrm{ml}$ of saline to clean the rectum. Five minutes thereafter, subjects were instructed to void rectal contents.

Subsequently, the volunteers laid down on a bed in a left lateral supine position and remained in this position during the entire test procedure. A commercially available barostat balloon (Mui Scientific, Ontario, Canada, part: C7-2CB-R) was lubricated with KY gel (Johnsson \& Johnsson, Langhorne, Pennsylvania) and inserted rectally $3 \mathrm{~cm}$ proximal to the anal sphincter. After a 5 minutes habituation period, the balloon was attached to the barostat equipment (Distender II, G\&J electronics, Ontario, Canada) and the barostat procedure was started. The controlled balloon distensions were programmed using the standard software package of the barostat equipment (Protocol Plus Deluxe, version 6_7; G\&J electronics, Ontario, Canada). 
The barostat protocol consisted of four sub-protocols, each designed for the measurement of specific parameters of interest:

\section{i) Balloon unfolding}

This part of the protocol consisted of 1 single distension at a balloon pressure of 20 $\mathrm{mmHg}$ for 1 minute, to ensure that the balloon was placed correctly without folds that may impair the airflow.

\section{ii) Minimal Distension Pressure (MDP)}

The second part of the protocol consisted of a staircase distension protocol with pressure steps of $1 \mathrm{mmHg}$ with a duration of 30 seconds each and a range from 0 $20 \mathrm{mmHg}$. The MDP, which is the minimal balloon pressure needed to overcome the intra-abdominal pressure, was defined as the first pressure at which respiratory curves were present in the volume recording of the balloon. The entire protocol was performed up to the $20 \mathrm{mmHg}$ pressure in all subjects as a sensitisation step prior to the compliance and perception measurements. The obtained MDP value was set to zero as a reference point. During this protocol the volunteers were asked to report the moment at which they could sense the balloon for the first time. This pressure was defined as the threshold for first sensation.

\section{iii) Compliance}

Directly after finishing the MDP measurements, the third part of the protocol was initiated. This part of the protocol, designed for compliance measurement, which represents the elasticity of the rectum in $\mathrm{ml} / \mathrm{mmHg}$, consisted of a staircase distension protocol with pressure steps of $3 \mathrm{mmHg}$ with a duration of 30 seconds each and a range of 0-33 $\mathrm{mmHg}$.

\section{iv) Visceral perception}

Subsequently, the distension protocol of the visceral perception measurements was initiated. This protocol consisted of semi-random distensions (at 4, 13, 10, 19, 16, 25, $22,31,28,37,34,43,40,49,46,55,52,61,58,67,64,71 \mathrm{mmHg}$ above MDP, respectively) with a duration of 1 minute each, interspaced with 30 second intervals at MDP. Thirty seconds after the start of each distension, volunteers scored the sensation of pain and discomfort on a $10 \mathrm{~cm}$ Visual Analogue Scale (VAS) and urge on a 6 -point scale $(0=$ no feeling, $1=$ just sensible , $2=$ clearly sensible/ light urge, $3=$ normal urge, $4=$ strong urge/ have to run to toilet, $5=$ maximum/stop) represented by 6 buttons on an electronic control panel (distender II perception panel) ${ }^{18,19}$, which was directly linked to the barostat equipment. The procedure was stopped when the maximum score for pain, urge or discomfort was reached. 


\section{Statistics}

The pain and discomfort data were analysed using a Gaussian non-linear regression, a random effect, and an autocorrelation. Urge was scored on an ordinal 6-point scale and was analyzed using a combination of a logistic distribution (parameterised as a proportional-odds) and a gamma distribution (to introduce a series dependence) ${ }^{20}$. The mean regression was imposed through the time variable to follow a logistic ('Sshape') curve. The model included pressure, MDP, first sensation, procedure (treated or not), and carry over effect as explanatory variables. The inference criterion used for comparing the models is their ability to predict the observed data, i.e. models are compared directly through their minimised minus log-likelihood. When the numbers of parameters in models differed, they were penalised by adding the number of estimated parameters, a form of the Akaike information criterion (AIC) ${ }^{21}$. The variable under consideration was found to be affected by butyrate if the AIC decreased compared to the model not containing the treatment. For each variable of interest, the dose as a continuous variable was then added to the model. Effects were considered significant if the AIC decreased and the confidence intervals did not overlap. The effect of butyrate on first sensation thresholds was considered significant when the confidence intervals of the threshold difference did not include zero.

\section{Results}

Eleven volunteers were enrolled in the study. One volunteer was excluded from further participation in the study due to non-compliance, which was assessed on basis of a questionnaire and the returned enema-bottles. No side effects of the enema use were reported and no carry-over was found between the three test conditions.

Butyrate treatment resulted in a significant reduction in pain, urge and discomfort scores over the entire pressure range of the protocol in a dose dependent way (Figure 4.2, 4.3 and Table 4.1). In Figure 4.2 also the confidence intervals for the pain scores are shown, demonstrating a significant effect of butyrate over the entire pressure range.

The confidence intervals for the discomfort scores were too small for graphical representation and therefore presented in Table 4.2, at three chosen points in the pressure range of the protocol (at 4, 22 and $40 \mathrm{mmHg}$ above MDP). The urge scores, shown in Table 4.1, also differed significantly over the entire pressure range. Due to the ordinal (6-point scale) characteristics it was not possible to provide confidence intervals of this parameter. For each dose of butyrate, the urge score with the highest likelihood to be scored at each pressure step, has been presented. 


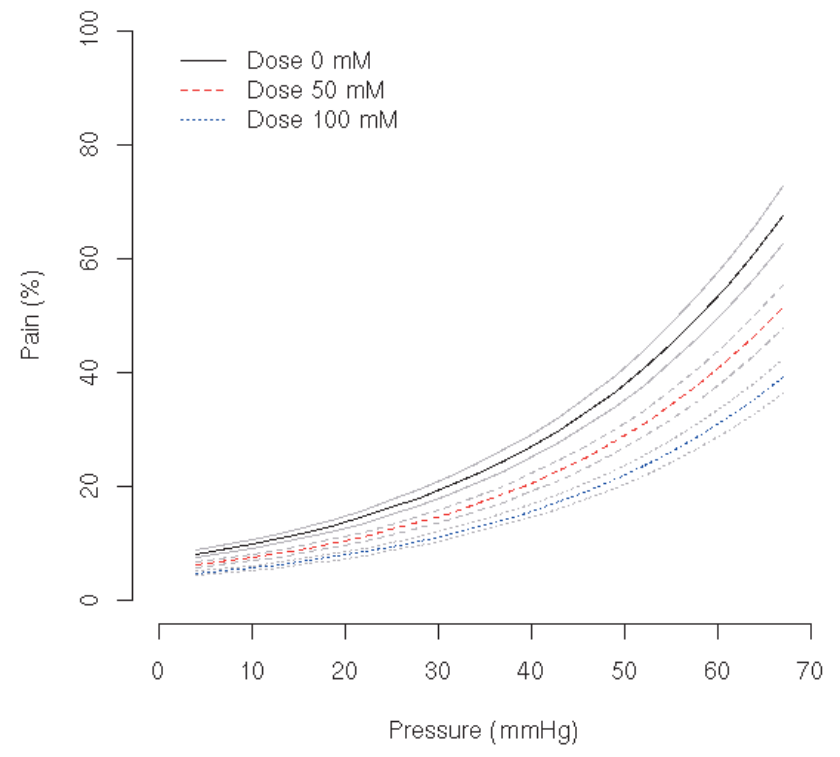

Figure 4.2 The effect of daily administration of enemas containing 0,50 or $100 \mathrm{mM}$ butyrate for 7 days on pain scores (100 mm VAS) at the consecutive pressure steps of the barostat protocol. $90 \%$ Confidence intervals of the pain scores are shown in grey.

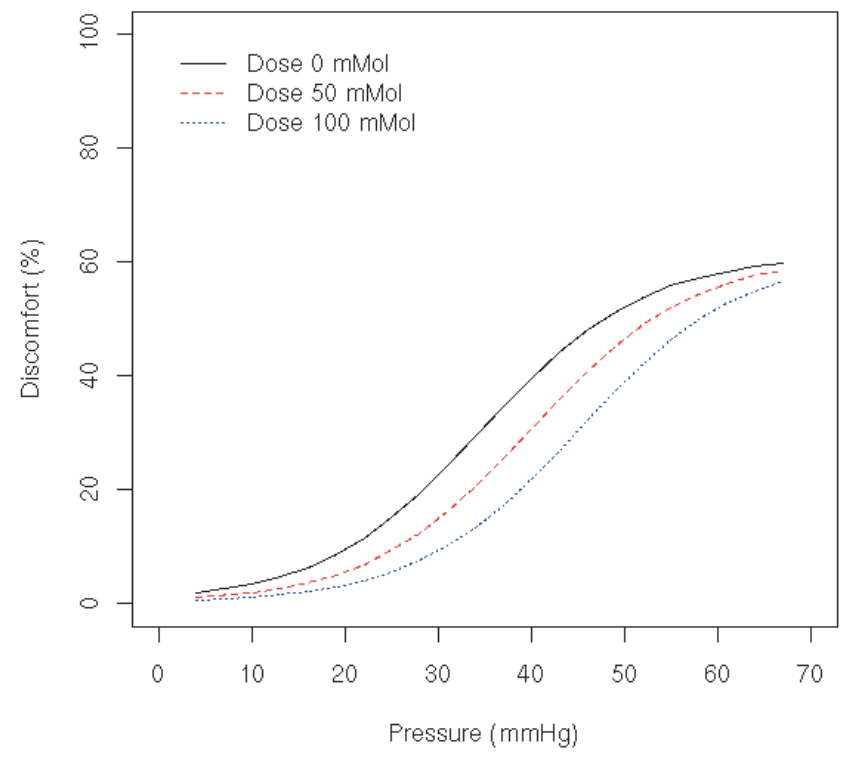

69

Figure 4.3 The effect of daily administration of enemas containing 0,50 and $100 \mathrm{mM}$ butyrate for 7 days on discomfort scores (100 mm VAS) at the consecutive pressure steps of the barostat protocol. 
Table 4.1 The effect of daily administration of enemas containing 0, 50 and $100 \mathrm{mM}$ butyrate for 7 days on urge scores (6-point scale) at the consecutive pressure steps of the barostat protocol.

\begin{tabular}{lccc}
\hline Pressure $(\mathrm{mmHg})$ & Dose 0 & Dose 50 & Dose 100 \\
\hline 4 & 1 & 0 & 0 \\
10 & 1 & 1 & 1 \\
13 & 1 & 1 & 1 \\
16 & 2 & 1 & 2 \\
19 & 2 & 2 & 2 \\
22 & 3 & 2 & 2 \\
25 & 3 & 3 & 3 \\
28 & 3 & 3 & 3 \\
31 & 3 & 3 & 3 \\
34 & 4 & 4 & 4 \\
37 & 4 & 4 & 4 \\
40 & 4 & 4 & 4 \\
43 & 4 & 4 & 4 \\
46 & 4 & 4 & 4 \\
49 & 4 & 4 & 4 \\
52 & 5 & 5 & 5 \\
55 & 5 & 5 & 5 \\
58 & 5 & 5 & 5 \\
61 & 5 & 5 & 5 \\
64 & 5 & 5 & 5 \\
67 & 5 & 5 & \\
\hline
\end{tabular}

Table 4.2 90\% Confidence intervals (upper and lower) of discomfort scores at the start, the middle and the end of the pressure range of the protocol, indicating a significant, dose dependent effect of butyrate.

\begin{tabular}{lccc}
\hline & Press $4 \mathrm{mmHg}$ & Press $22 \mathrm{mmHg}$ & Press $40 \mathrm{mmHg}$ \\
\hline $0 \mathrm{mM}$ butyrate & $1.86-1.88$ & $11.35-11.46$ & $39.46-39.63$ \\
$50 \mathrm{mM}$ butyrate & $1.035-1.05$ & $6.81-6.89$ & $30.60-30.79$ \\
$100 \mathrm{mM}$ butyrate & $0.57-0.58$ & $3.95-4.0$ & $21.74-21.91$ \\
\hline
\end{tabular}

The pressure threshold for first sensation was higher after the placebo treatment compared to baseline (increase of $3 \mathrm{mmHg} \mathrm{Cl} \mathrm{0,87-5,12} \mathrm{mmHg}$ ). Compared to placebo, both 50 and $100 \mathrm{mM}$ of butyrate administration significantly reduced the threshold (reduction of 2,7; $\mathrm{Cl}$ 0,11-5,33 $\mathrm{mmHg}$ and 4,9; $\mathrm{Cl} 2,18-7,65 \mathrm{mmHg}$ respectively).

Only the highest dosage of butyrate increased the dynamic compliance, when measured at a pressure of $12 \mathrm{mmHg}$, which was in the linear part of the graph (Figure 4.4). At a pressure of $12 \mathrm{mmHg}$, the volume increased from $200 \mathrm{ml}$ (Cl 197-203 ml) after placebo and $50 \mathrm{mM}$ butyrate to $215 \mathrm{ml}(\mathrm{Cl} 212-218 \mathrm{ml}$ ) after $100 \mathrm{mM}$. 


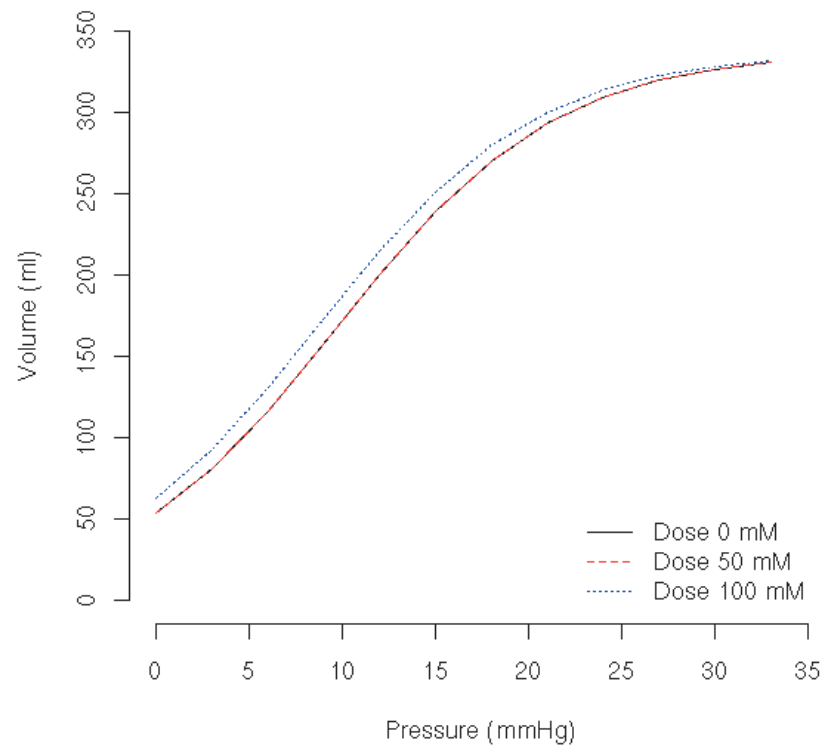

Figure 4.4 Pressure-Volume relationship (compliance) for each dose of butyrate. No difference was found between 0 and $50 \mathrm{mM}$.

\section{Discussion}

The aim of the present study was to evaluate the physiological effects of butyrate on visceral perception in healthy subjects. The study showed that one week rectal butyrate administration decreased the perception of pain, urge and discomfort compared to placebo in a dose dependent fashion. Compliance of the rectal wall increased significantly after $100 \mathrm{mM}$ butyrate treatment.

The present results indicating a significant decrease in visceral perception are in contrast with previous findings from rat studies, in which butyrate prolonged visceral hypersensitivity in TNBS-treated rats and induced visceral hypersensitivity in control animals $^{16,17}$. Although the perception scores were decreased due to the butyrate treatment, no conclusions about the putative underlying mechanisms can be drawn since we did not take biopsy samples for histological, biochemical and genetic analysis.

The decrease in visceral perception due to butyrate treatment could be via modulation of 5-hydroxytryptamine (5-HT or serotonin) activity. 5-HT is a neurotransmitter that is found predominantly in the gastrointestinal tract. SCFAs are known to stimulate intraluminal 5-HT release in rat colon, resulting in an increase in motility ${ }^{22}$. Previous studies in humans have shown that an increased $5-\mathrm{HT}$ release 
leads to an increased compliance and a decrease in visceral perception ${ }^{18}$. 5-HT exerts its actions by activation of specific serotonin receptors, of which $5-\mathrm{HT}_{3}$ and $5-\mathrm{HT}_{4}$ are known to induce colonic contractions leading to an increased motility ${ }^{23}$ and some evidence exists for a neuroprotective effect of $5-\mathrm{HT}_{4}$ receptor stimulation ${ }^{24}$. The effect of serotonin on visceral sensitivity is not clear but an increased compliance has been reported in literature, suggesting that sensation thresholds may be higher when serotonin is released ${ }^{25-27}$.

An indirect effect of the butyrate-mediated 5-HT release could be through sensitisation of transient receptor potential vanilloid 1 (TRPV1) receptors in the colonic mucosa. TRPV1 receptors are responsible for pain transduction to the central nervous system and stimulation of these receptors by butyrate or 5-HT could lead to an increase in pain sensation. Moreover, increased TRPV1 receptor expression in IBS was found to correlate with abdominal pain ${ }^{28}$. Overstimulation or repetitive stimulation of this receptor however, is known to desensitise afferent neurons as has been shown for capsaicin ${ }^{29,30}$. Since butyrate-induced serotonin release could trigger TRPV1, it may well be that desensitisation of TRPV 1 after one week of butyrate administration occurs. These effects are in concordance with the effects of butyrate on perception scores and compliance in the present study.

Another mechanism by which butyrate could affect visceral perception is via inhibition of histone deacetylase (HDAC). Several of these inhibitors, valproate, butyrate and trichostatin A, have previously been reported to induce microglyal apoptosis and to reduce inflammation-induced neurotoxicity in rat tissue, which may affect visceral perception $^{31}$. Those findings, together with the previously reported observation that valproate is effective in pain reduction ${ }^{32}$ suggest that butyrate exerts its effect on visceral sensitivity in part via its histon deacetylation inhibiting capacity.

These possible mechanisms underlying the effect of butyrate on visceral perception do not fully explain the difference between the results found in rats and humans. However, the differences may in part be explained by the concentration differences or differences between 5 -HT receptor subtypes and their precise function and location in both species. Furthermore, the methods for the visceral perception measurements in rats (behavioural changes and abdominal contractions) and humans (VAS or ordinal scales) are different, which makes a good comparison of the results difficult. The subjects of this study were well instructed and underwent a dummy barostat measurement at the screening to ensure a minimal level of uncertainty and anxiety. This may further explain the difference between well-instructed volunteers and rats, since fear and anxiety have been reported to influence pain scores ${ }^{33-35}$.

The applied barostat methodology is a generally accepted and validated method to measure visceral perception in humans. The pressures that were induced during the semi-random staircase protocol for visceral perception were corrected for the minimal distension pressure (MDP). In the present study, the MDP value was determined by increasing the pressure gradually until respiratory waves were visible in the volume curve of the barostat apparatus. Although we are aware of alternative 
methods to estimate $\mathrm{MDP}^{36}$, we consider this approach as most reliable as it is based on physiological differences between subjects and conditions rather than an arbitrary cut-off.

In a previous pilot study the length of the wash-out period of two weeks was determined and found to be sufficient (data not shown). This was confirmed by the present study as no carry-over effect of butyrate and placebo was found.

In the present study the butyrate and placebo were delivered by enemas, which provide a reliable and non-invasive tool to deliver a substance to the distal colon. Although this way of delivery was considered most appropriate, a placebo effect was found on the parameter "first sensation" (FS), which may have been caused by the use of enemas. None of the other parameters was significantly affected by placebo administration.

The results of the present study show a remarkable improvement of parameters of visceral perception and suggest a possible beneficial effect of butyrate in disorders, which are associated with visceral hypersensitivity such as Irritable Bowel Syndrome (IBS). This is in line with previous findings of Kilkens et al., which demonstrate a serotonin-dependent difference on compliance between IBS patients and controls ${ }^{18}$. More research is needed to unravel the mechanisms of action by which butyrate decreases visceral perception. Furthermore, the results of the present study should be confirmed by an oral-intake study with non-digestible fibers, which lead to a fermentation mediated increase colonic butyrate levels.

In conclusion, intra-luminal administration of a physiologically relevant dose of butyrate into the distal colon, increases compliance and decreases pain, urge and discomfort measured with a rectal barostat procedure in healthy subjects. This provides a basis for future trials with dietary modulation resulting in intra-colonic butyrate production in both healthy and IBS subjects. 


\section{References}

1. Bergman EN. Energy contributions of volatile fatty acids from the gastrointestinal tract in various species. Physiological reviews. 1990;70:567-90.

2. Hamer HM, Jonkers D, Venema K, Vanhoutvin S, Troost FJ, Brummer RJ. Review article: the role of butyrate on colonic function. Aliment Pharmacol Ther. 2008;27:104-19.

3. Kanauchi O, Iwanaga T, Andoh A, Araki Y, Nakamura T, Mitsuyama K, et al. Dietary fiber fraction of germinated barley foodstuff attenuated mucosal damage and diarrhea, and accelerated the repair of the colonic mucosa in an experimental colitis. J Gastroenterol Hepatol. 2001;16:160-8.

4. Jacobasch G, Schmiedl D, Kruschewski M, Schmehl K. Dietary resistant starch and chronic inflammatory bowel diseases. Int J Colorectal Dis. 1999;14:201-11.

5. Tsukahara T, Iwasaki Y, Nakayama K, Ushida K. Stimulation of butyrate production in the large intestine of weaning piglets by dietary fructooligosaccharides and its influence on the histological variables of the large intestinal mucosa. Journal of nutritional science and vitaminology. 2003; 49:414-21.

6. Fuller R, Perdigon G. Gut Flora, Nutrition, Immunity and Health: Plackwell Publishing 2003.

7. Boren J, Lee WN, Bassilian S, Centelles JJ, Lim S, Ahmed S, et al. The stable isotope-based dynamic metabolic profile of butyrate-induced HT29 cell differentiation. J Biol Chem. 2003;278:28395-402.

8. Ogawa H, Rafiee P, Fisher PJ, Johnson NA, Otterson MF, Binion DG. Butyrate modulates gene and protein expression in human intestinal endothelial cells. Biochem Biophys Res Commun. 2003;309:512-9.

9. Gaudier E, Jarry A, Blottiere HM, de Coppet P, Buisine MP, Aubert JP, et al. Butyrate specifically modulates MUC gene expression in intestinal epithelial goblet cells deprived of glucose. Am J Physiol Gastrointest Liver Physiol. 2004;287:G1168-G74.

10. Willemsen LE, Koetsier MA, van Deventer SJ, van Tol EA. Short chain fatty acids stimulate epithelial mucin 2 expression through differential effects on prostaglandin $E(1)$ and $E(2)$ production by intestinal myofibroblasts. Gut. 2003;52:1442-7.

11. Blache D, Gesquiere L, Loreau N, Durand P. Oxidant stress: the role of nutrients in cell-lipoprotein interactions. Proc Nutr Soc. 1999;58:559-63.

12. Rosignoli P, Fabiani R, De Bartolomeo A, Spinozzi F, Agea E, Pelli MA, et al. Protective activity of butyrate on hydrogen peroxide-induced DNA damage in isolated human colonocytes and HT29 tumour cells. Carcinogenesis. 2001;22:1675-80.

13. Kvietys PR, Granger DN. Effect of volatile fatty acids on blood flow and oxygen uptake by the dog colon. Gastroenterology. 1981;80:962-9.

14. Bordin M, D'Atri F, Guillemot L, Citi S. Histone deacetylase inhibitors up-regulate the expression of tight junction proteins. Mol Cancer Res. 2004;2:692-701.

15. Venkatraman A, Ramakrishna BS, Shaji RV, Kumar NS, Pulimood A, Patra S. Amelioration of dextran sulfate colitis by butyrate: role of heat shock protein 70 and NF-kappaB. Am J Physiol Gastrointest Liver Physiol. 2003;285:G177-84.

16. Bourdu S, Dapoigny M, Chapuy E, Artigue F, Vasson MP, Dechelotte P, et al. Rectal instillation of butyrate provides a novel clinically relevant model of noninflammatory colonic hypersensitivity in rats. Gastroenterology. 2005;128:1996-2008.

17. Tarrerias AL, Millecamps M, Alloui A, Beaughard C, Kemeny JL, Bourdu S, et al. Short-chain fatty acid enemas fail to decrease colonic hypersensitivity and inflammation in TNBS-induced colonic inflammation in rats. Pain. 2002;100:91-7.

18. Kilkens TO, Honig A, van Nieuwenhoven MA, Riedel WJ, Brummer RJ. Acute tryptophan depletion affects brain-gut responses in irritable bowel syndrome patients and controls. Gut. 2004;53:1794-800.

19. Kilkens TO, Honig A, Fekkes D, Brummer RJ. The effects of an acute serotonergic challenge on braingut responses in irritable bowel syndrome patients and controls. Aliment Pharmacol Ther. 2005;22: 865-74.

20. Lindsey PJ, Kaufmann J. Analysis of a longitudinal ordinal response clinical trial using dynamic models. Journal of the Royal Statistical Society Series C-Applied Statistics. 2004;53:523-37.

21. Akaike H. Information theory and an extension of the maximum likelihood principle. Second international symposium on inference theory (Tsahkadsor, 1971). 1973:267-81. 
22. Fukumoto S, Tatewaki M, Yamada T, Fujimiya M, Mantyh C, Voss M, et al. Short-chain fatty acids stimulate colonic transit via intraluminal 5-HT release in rats. Am J Physiol Regul Integr Comp Physiol. 2003;284:R1269-76.

23. Camilleri M, Von der Ohe MR. Drugs affecting serotonin receptors. Bailliere's clinical gastroenterology. 1994;8:301-19.

24. Gershon MD, Liu MT. Serotonin and neuroprotection in functional bowel disorders. Neurogastroenterol Motil. 2007;19 Suppl 2:19-24.

25. Tack J, Broekaert D, Corsetti M, Fischler B, Janssens J. Influence of acute serotonin reuptake inhibition on colonic sensorimotor function in man. Aliment Pharmacol Ther. 2006;23:265-74.

26. Delvaux M, Louvel D, Mamet JP, Campos-Oriola R, Frexinos J. Effect of alosetron on responses to colonic distension in patients with irritable bowel syndrome. Aliment Pharmacol Ther. 1998;12: 849-55.

27. Greenwood-van Meerveld B. Importance of 5-hydroxytryptamine receptors on intestinal afferents in the regulation of visceral sensitivity. Neurogastroenterol Motil. 2007;19 Suppl 2:13-8.

28. Akbar A, Yiangou Y, Facer P, Walters JR, Anand P, Ghosh S. Increased capsaicin receptor TRPV1expressing sensory fibres in irritable bowel syndrome and their correlation with abdominal pain. Gut. 2008;57:923-9.

29. Holzer P. The pharmacological challenge to tame the transient receptor potential vanilloid-1 (TRPV1) nocisensor. Br J Pharmacol. 2008;155:1145-62.

30. Holzer P. TRPV1: a new target for treatment of visceral pain in IBS? Gut. 2008;57:882-4

31. Chen PS, Wang CC, Bortner CD, Peng GS, Wu X, Pang H, et al. Valproic acid and other histone deacetylase inhibitors induce microglial apoptosis and attenuate lipopolysaccharide-induced dopaminergic neurotoxicity. Neuroscience. 2007;149:203-12.

32. Kochar DK, Jain N, Agarwal RP, Srivastava T, Agarwal P, Gupta S. Sodium valproate in the management of painful neuropathy in type 2 diabetes - a randomized placebo controlled study. Acta neurologica Scandinavica. 2002;106:248-52.

33. Rhudy JL, Meagher MW. Negative affect: effects on an evaluative measure of human pain. Pain. 2003; 104:617-26.

34. Rhudy JL, Meagher MW. Fear and anxiety: divergent effects on human pain thresholds. Pain. 2000;84: 65-75.

35. Staats PS, Staats A, Hekmat H. The additive impact of anxiety and a placebo on pain. Pain medicine (Malden, Mass. 2001;2:267-79.

36. van den Berg MM, Voskuijl WP, Boeckxstaens GE, Benninga MA. Rectal compliance and rectal sensation in constipated adolescents, recovered adolescents and healthy volunteers. Gut. 2008;57:599-603. 

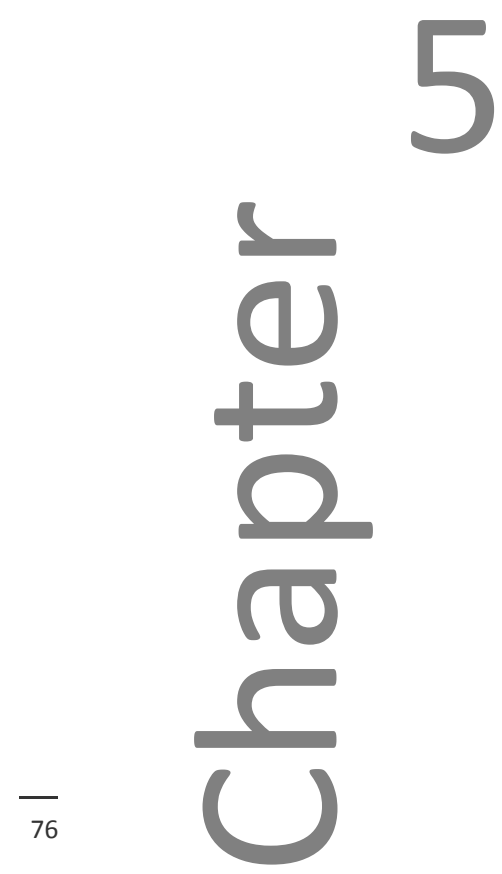


\section{Rectal butyrate instillation decreases visceral perception in IBS patients}

Steven ALW Vanhoutvin, Freddy J Troost, Daisy MAE Jonkers, Patrick J Lindsey, Henrike M Hamer, Koen Venema, Ger Koek Robert JM Brummer, Ad AM Masclee 


\section{Abstract}

\section{Background}

Irritable Bowel Syndrome (IBS) is a functional bowel disorder with a high prevalence, affecting up to $15 \%$ of the general population. Visceral hypersensitivity is a hallmark that has been reported in up to $60 \%$ of IBS patients. We have previously shown that the short chain fatty acid butyrate after rectal administration is able to significantly reduce visceral sensitivity in healthy controls.

Aim of this study was to determine the effects of rectally administered butyrate on visceral perception in IBS patients.

\section{Methods}

16 IBS patients (Rome III, mean age $40 \pm 14$ y, 6 males) participated in a randomized double blind placebo controlled cross-over study with rectal enemas containing either butyrate (100 mM) or placebo (saline) once daily prior to sleeping, for one week. Visceral perception and compliance were assessed at the start and the end of each test week during a rectal barostat procedure. In a separate ex vivo experiment in Ussing chambers, the potential protective effect of different concentrations of butyrate on colonic permeability was studied after challenge with the bile acid deoxycholate.

\section{Results}

Daily administration of $100 \mathrm{mM}$ butyrate enemas for one week resulted in a significant reduction in the perception of urge and pain, but did not affect rectal compliance. The Ussing chamber experimental data indicate that butyrate beneficially affects colonic permeability. The changes in permeability and perception induced by butyrate may be mutually related.

\section{Conclusions}

Rectocolonic butyrate administration for one week reduces pain and urge sensations during rectal mechanical distension in IBS patients. Part of this effect may have been obtained via changes in gut permeability. Consumption of non-digestible carbohydrates, resulting in colonic production of butyrate due to microbial fermentation, may provide novel dietary strategies for treatment of (visceral hypersensitivity in) IBS patients. 


\section{Introduction}

Irritable Bowel Syndrome (IBS) is a functional bowel disorder with a high prevalence affecting up to $15 \%$ of the general population ${ }^{1,2}$. It is a heterogeneous and multifactorial disorder, characterised by abdominal symptoms and alterations in bowel habits. Visceral hypersensitivity is an important hallmark present in up to $64 \%$ of IBS patients ${ }^{3}$ and is associated with more pronounced daily symptoms, reduced quality of life, anxiety disorder and depression ${ }^{4}$. IBS symptoms are typically provoked or aggravated by meal intake or by specific nutrients such as lactose or fructose, fat or fatty acids. While specific nutrients provoke symptoms, other nutrients or substances may provide relief. The use of dietary ingredients that modify visceral perception and thereby may result in reduction of daily symptoms and complaints in IBS patients may provide a new therapeutic strategy in IBS. In this respect, non-digestible carbohydrates may have therapeutic potential since ingestion of these carbohydrates results in an increased colonic production of short chain fatty acids (SCFA), such as acetate, propionate and butyrate, due to saccharolytic fermentation by colonic microbiota. SCFA are rapidly absorbed and metabolized by the gut mucosa and contribute to mucosal homeostasis and intestinal barrier function. The SCFA butyrate is involved in regulation of various processes such as cell proliferation ${ }^{5}$, mucosal antioxidant capacity ${ }^{6}$, inflammation through cytokine production and myeloperoxidase

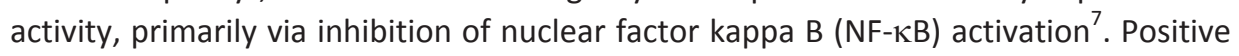
effects of butyrate on intestinal inflammation have been reported not only in animal models of colitis but also in patients with active ulcerative colitis ${ }^{8,9}$. Finally, butyrate may beneficially affect intestinal permeability ${ }^{10}$, a key factor that has repeatedly been associated with both visceral hypersensitivity and $\mathrm{IBS}^{11-14}$. Treatment with prebiotic enzyme-treated rice fiber resulted in increased levels of intestinal butyrate and prevented the occurrence of visceral hypersensitivity in a rat model of IBS ${ }^{15}$. We have shown previously that repeated administration of butyrate via enemas resulted in a significant decrease in visceral perception in healthy volunteers ${ }^{16}$. That finding raised the question whether local application of butyrate to the distal colon will also affect visceral perception in conditions with a high prevalence of visceral hypersensitivity, such as IBS ${ }^{17}$. The objective of the present study was to evaluate the effect of colonic administered butyrate on visceral perception in IBS patients. We hypothesised that daily rectal administration of butyrate over a one-week period will decrease visceral perception in IBS patients, in line with our previous observation in healthy volunteers. Since visceral hypersensitivity and symptoms in IBS are associated with impaired intestinal barrier function, we also evaluated in an ex-vivo model the potential role of butyrate to reduce a stressor-induced increase in colonic mucosal permeability in human colonic tissue obtained from healthy controls. 


\section{Materials and methods}

\section{In vivo barostat experiment}

\section{Subjects}

Sixteen IBS patients ( 6 males and 10 females, mean age $40 \pm 14$ years), diagnosed by the Rome III criteria were enrolled in this study. Seven of the IBS patients had diarrhea-predominant, six constipation-predominant and three had mixed type of IBS (Table 5.1). The study was approved by the Medical Ethics Committee of Maastricht University Medical Center and was conducted in accordance with the principles of the 'Declaration of Helsinki' (52nd WMA General Assembly, Edinburgh, Scotland, Oct 2000). All volunteers gave written informed consent before participation. The study has been registered in the US National Library of Medicine (http://www.clinicaltrials.gov, NCT00696098)

Table 5.1 Patient characteristics and IBS sub type.

\begin{tabular}{|c|c|c|c|}
\hline NR & Gender & Age & IBS-type \\
\hline 1 & $M$ & 46 & D \\
\hline 2 & V & 26 & c \\
\hline 3 & $M$ & 62 & $d$ \\
\hline 4 & $M$ & 49 & $d$ \\
\hline 5 & $M$ & 53 & $d$ \\
\hline 6 & V & 28 & $d$ \\
\hline 7 & V & 54 & mixed \\
\hline 8 & V & 20 & c \\
\hline 9 & $M$ & 33 & c \\
\hline 10 & V & 48 & c \\
\hline 11 & V & 54 & c \\
\hline 12 & V & 26 & mixed \\
\hline 13 & V & 26 & $d$ \\
\hline 14 & V & 41 & $d$ \\
\hline 15 & M & 21 & c \\
\hline 16 & $\mathrm{~V}$ & 57 & mixed \\
\hline
\end{tabular}

\section{Study design}

This randomised, double blind, placebo-controlled cross-over study consisted of two test periods of one week each, with a wash-out period of two weeks in between. The duration of the wash-out period was based on the results of a previous study in healthy volunteers showing no carry-over effect with two weeks wash-out ${ }^{16}$. The patients self-administered rectal enemas containing a $60 \mathrm{ml}$ solution of either butyrate $(100 \mathrm{mM})$ or placebo $(0.9 \% \mathrm{NaCl})$ once daily prior to sleeping. Patients were instructed to stay in a left lateral supine position to ensure optimal dispersion of the test solutions in the distal colon during the first 20 min after enema instillation. The 
enemas were made isotonic with sodium chloride and had a pH of 7. Rectal barostat measurements to assess visceral perception were performed at the start and at the end of each of the two test weeks. Compliance of the enema administration, stool frequency and fecal consistency according to the Bristol Stool form scale and abdominal symptoms (flatulence, bloating, abdominal cramps) were monitored daily using a questionnaire. Abdominal complaints were scored as mild (1) moderate (2) or severe (3) as was previously described by Hamer et al. ${ }^{18}$. Subjects were instructed to maintain their regular dietary habits during both test weeks.

\section{Barostat protocol}

The barostat protocol applied in this study was identical to the one described previously in the study with healthy volunteers ${ }^{16}$. All subjects underwent a dummy barostat measurement prior to enrollment to become familiar with the barostat procedure. On each test day, after arrival at the hospital, the patients selfadministered a rectal enema containing $60 \mathrm{ml}$ of saline and voided after $5 \mathrm{~min}$, to clear rectal contents. Subsequently, patients remained in a left lateral supine position during the entire test procedure. A commercially available barostat balloon (MUI Scientific, C7-2CB-R, Ontario, Canada) was lubricated with KY gel (Johnsson \& Johnsson, Langhorne, Pennsylvania) and inserted rectally $3 \mathrm{~cm}$ proximal to the anal sphincter. After a 5-min habituation period, the balloon was attached to the barostat equipment (Distender II, G\&J electronics, Ontario, Canadan, controlled by the software Protocol Plus Deluxe, version 6_7; G\&J electronics, Ontario, Canada) and the barostat procedure was initiated. The barostat protocol consisted of four parts. During the first part of the protocol, the barostat balloon was unfolded by a single pressure step. In the second part of the protocol, first sensation (FS) and minimal distension pressure (MDP) were assessed during a staircase procedure. This part of the protocol was performed up to the $20 \mathrm{mmHg}$ pressure as a sensitisation step prior to the subsequent compliance and perception measurements. Subsequently, rectal compliance was determined, which was corrected for MDP. Finally, visceral perception was determined using a semi-random staircase protocol as described previously ${ }^{16}$.

\section{Ex vivo Ussing chamber experiment}

\section{Biopsy samples}

Biopsy samples for Ussing chamber experiments were obtained from eleven healthy volunteers ( 6 females, age 18 to 54yrs). Exclusion criteria were gastrointestinal disease or surgery, age over 65 years, and use of any medication, probiotics or prebiotics three months prior to inclusion. All participants signed an informed consent prior to participation to the study, which was approved by the Ethical Committee of the University Hospital Maastricht, the Netherlands, and conducted in full accordance 
with the principles of the 'Declaration of Helsinki' $\left(52^{\text {nd }}\right.$ WMA General assembly, Edinburgh, Scotland, Oct 2000).

Subjects arrived at the endoscopy unit in the morning and were asked to void stool prior to undergoing sigmoidoscopy. Clear drinks were allowed ad libitum during the $10 \mathrm{~h}$ preceding the examination. During sigmoidoscopy without analgesia or sedation, tissue samples were obtained from the unprepared sigmoid (no laxatives or enemas allowed). In each subject eight tissue samples were taken $20-25 \mathrm{~cm}$ above the anal sphincter. Biopsies were preserved immediately in cold oxygenated modified KrebsRinger buffer (KRB) and arrived at the laboratory facility within 15 min after sampling.

\section{Ussing chamber procedure}

The tissue samples were mounted in modified $1.5 \mathrm{ml}$ Ussing Chambers (Harvard Apparatus Inc., Holliston, Mass., USA) with an opening of 9-mm in diameter and reduced to an exposed tissue area of $1.76 \mathrm{~mm}^{2}$, using a technique previously described by Wallon et al. After mounting, each half chamber was filled with $1.5 \mathrm{ml}$ $\mathrm{KRB}$, bathing both the mucosal and serosal side of the specimen. The KRB solution $(\mathrm{pH}$ 7.4) was continuously oxygenated and stirred by gas flow in the chambers and kept at a temperature of $37^{\circ} \mathrm{C}$ with a heater block system.

After a 20-min equilibration period to achieve steady-state conditions in electrophysiological parameters, the KRB was replaced in both compartments with fresh $\mathrm{KRB}$, containing different concentrations of butyrate $(0 \mathrm{mM}, 5 \mathrm{mM}, 10 \mathrm{mM}$, and $20 \mathrm{mM}$, respectively), in the mucosal compartment only. After another $20 \mathrm{~min}$ incubation period, the KRB solution from the mucosal compartment, except the solution in the negative control compartment, was replaced with $1 \mathrm{mM}$ deoxycholic acid, a potent stressor to induce an increased permeability ${ }^{19}$. In order to evaluate the macromolecular permeability, $1 \mathrm{mg} / \mathrm{ml}$ sucralose was added to the mucosal side. Electric potential difference (PD), transmucosal electrical resistance (TER) and shortcircuit current (Isc) were determined during the $60 \mathrm{~min}$ following addition of deoxycholic acid. Experiments were performed in open-circuit conditions with assessment of electrophysiological parameters at 2-min intervals. A sample of $0.3 \mathrm{ml}$ was taken at the start of addition of deoxycholic acid, and at 30 and $60 \mathrm{~min}$ thereafter, respectively from the serosal side and replaced with $0.3 \mathrm{ml}$ fresh KRB. Details on the Ussing chamber procedure are described elsewhere ${ }^{20}$.

\section{Sucralose analysis}

Sucralose concentrations were determined by liquid chromatography in combination with mass spectometry (LC-MS). Chromatographic separation was based on isocratic elution of sucralose on an IOA-10009 $\mu \mathrm{m}$ cation-exchange column (300 mmx7.8 mm ID; Grace, Deerfield, IL), mounted in a Mistral column oven (Separations, H.I. Ambacht, the Netherlands) at $30^{\circ} \mathrm{C}$. Samples and standards were injected using a 
Model 233XL sample processor with Peltier chilled sample storage compartments $\left(10^{\circ} \mathrm{C}\right)$, equipped with a $20 \mu \mathrm{l}$ sample loop (Gilson, Middleton, WI). MS detection was performed using a model LTQ XL (Thermo Fisher Scientific, Waltham, MA) equipped with an ion-Max electrospray probe. Details on this analytical procedure are provided elsewhere $^{21}$.

\section{Statistics}

The pain and discomfort data, expressed as percentage of the $100 \mathrm{~mm}$ VAS scale, were analysed using a multivariate Gaussian non-linear regression including a random effect and an autocorrelation as described previously ${ }^{16}$. Urge, scored on an ordinal 6point scale, was analyzed using a mixture of a logistic distribution (parameterised as a proportional-odds) and a gamma distribution (to introduce a frailty dependence) ${ }^{22}$. Mean scores of cramping, gas and bloating and of fecal frequency and consistency was calculated for the three days prior to each test day. Cramping, gas, and bloating complaints as well as the fecal consistency type were analysed using a paired Student's t-test. Stool frequency was analysed using a mixture of gamma distributions to introduce a serial dependence ${ }^{23}$. The Akaike Information Criterion (AIC) was used to assess whether there was a (butyrate) treatment effect. Intestinal compliance data were analysed using a multivariate Gaussian non-linear regression including two random effects. The AIC was used to assess whether there was a (butyrate) treatment effect.

An extensive description of the statistics used is provided in the supplementary material (S1).

Differences were considered significant at $\mathrm{P}<0.05$ or when the $95 \%$ Confidence intervals showed no overlap (in the multivariate Gaussian non-linear regression analysis).

For the Ussing chamber data, differences between chambers for TER and ISC were analysed by a mixed-effects model, with fixed effects for time and chamber and a random intercept per biopsy within subject. Baseline values were entered as an additional (fixed effect) covariate. F-tests were used to test significance of factors.

Differences in markerflux at $T=60$ minutes between the control and the test conditions were analysed non parametricly using the Wilcoxon rank sum test.

\section{Results}

\section{In vivo barostat experiment}

Out of the 16 IBS patients enrolled in the study, 15 completed the experiment. One patient was withdrawn 2 days after start of the treatment period with butyrate, because of abdominal pain and diarrhea. This patient was excluded from the data 
analysis. No side effects have been reported in the other 15 patients. All patients complied to the study protocol, based on information from daily questionnaires and the number of returned empty enema vials.

Instillation with $100 \mathrm{mM}$ butyrate enemas for one week resulted in a significant reduction of pain (Figure 5.1) and urge (Figure 5.2) but not of discomfort scores (Figure 5.3). For the pain scores, the differences between butyrate and placebo were significant throughout the entire pressure range of the barostat protocol, as indicated in the figures by the confidence intervals. Due to the ordinal characteristics of the urge scores, it was not possible to provide confidence intervals for this parameter. For each intervention (placebo or $100 \mathrm{mM}$ butyrate), the urge score with the highest likelihood to be scored at each pressure step has been presented (Figure 5.2), demonstrating a significant reduction of urge after butyrate treatment. The butyrate treatment did not have a significant effect on discomfort. Butyrate did not affect first sensation, rectal compliance or MDP. A carry-over effect of butyrate was found for both the variables discomfort and pain, but not for other parameters. This carry-over effect was corrected for by the statistical model, as the baseline measurements obtained at the start of each intervention period, were taken into account in the statistical analysis.

The intervention with butyrate did not significantly affect the score for stool type (Bristol Stool Scale) or any of the abdominal symptoms (i.e cramping, gas or bloating)

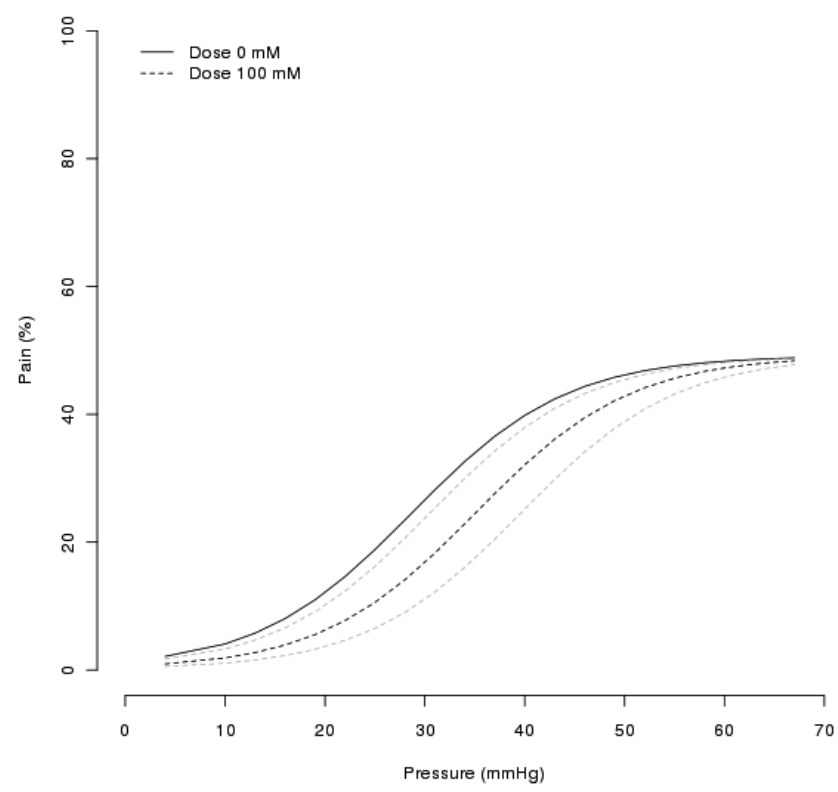

Figure 5.1 The effect of daily administration of enemas containing 0 or $100 \mathrm{mmol} \mathrm{L}^{-1}$ butyrate for 7 days on pain scores (100 mm VAS) at the consecutive pressure steps of the barostat protocol. 


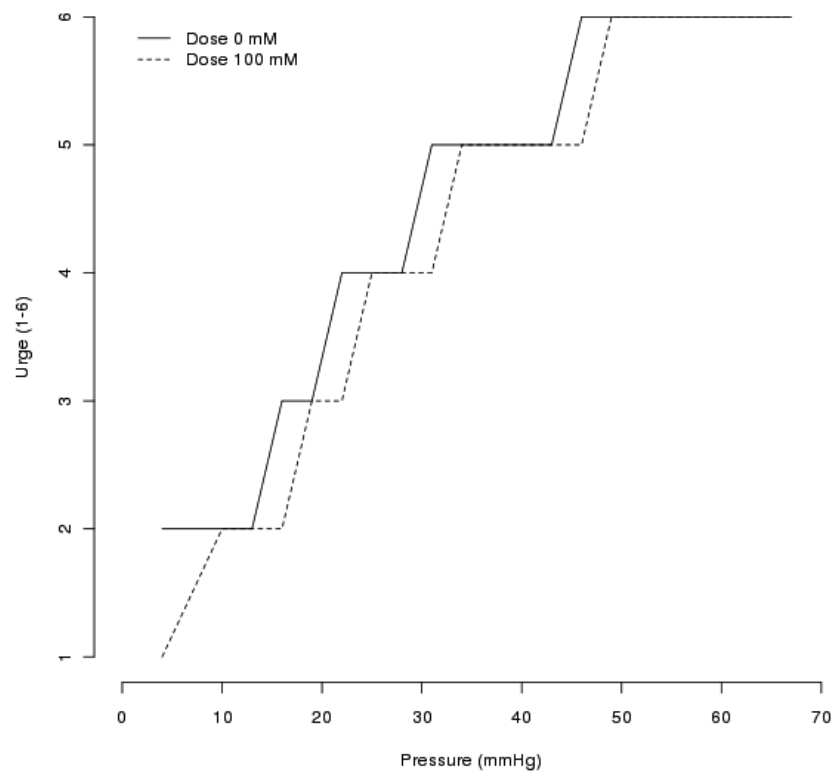

Figure 5.2 The effect of daily administration of enemas containing 0 or $100 \mathrm{mmol} \mathrm{L}^{-1}$ butyrate for 7 days on urge probablity scores (six-point scale) at the consecutive pressure steps of the barostat protocol.

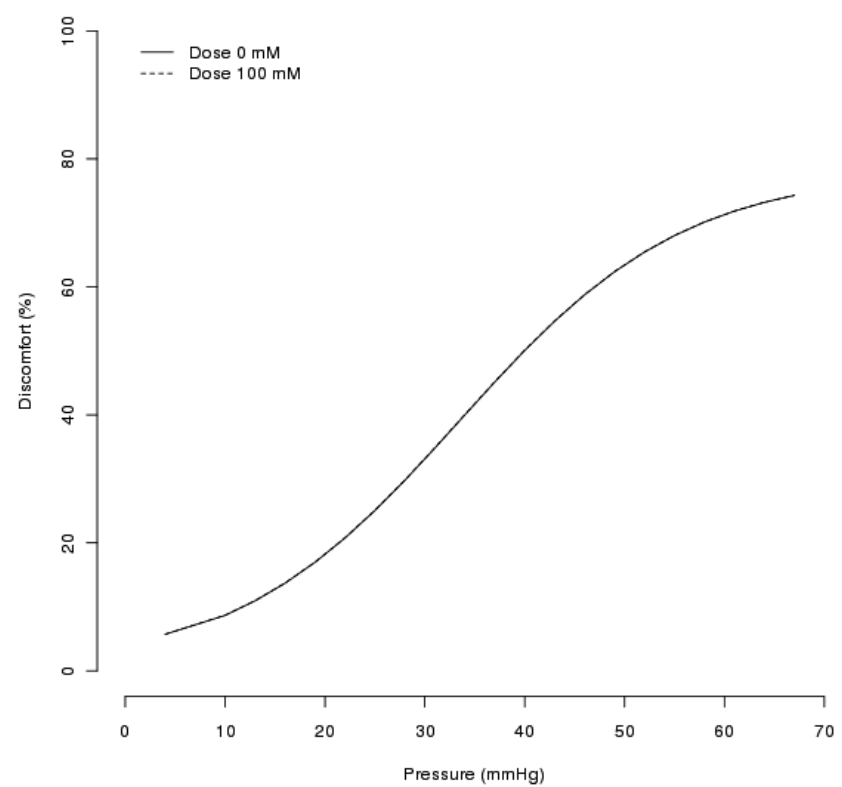

Figure 5.3 The effect of daily administration of enemas containing 0 or $100 \mathrm{mmol} \mathrm{L}^{-1}$ butyrate for 7 days on discomfort scores (100 mm VAS) at the consecutive pressure steps of the barostat protocol. 


\section{Ex vivo Ussing chamber experiments}

Viability of the tissue over the 60 min study period was confirmed by continuous monitoring of the PD between the two Ussing chamber compartments.

Compared to the negative control, addition of $1 \mathrm{mM}$ of deoxycholate (control) significantly increased sucralose flux at $t=60$ minutes from median $0.45(0.13-2.4)$ to median $2.17(0,37-15) ; \mathrm{P}=0.02$ (Figure 5.4). After correction for multiple testing this significance however was lost. Pretreatment with increasing concentrations of 5, 10 or $20 \mathrm{mM}$ of butyrate dose dependently decreased the deoxycholate induced increase of sucralose flux although the differences did not reach statistical significance $(P=0,87$; 0,49 and 0,28 , respectively)

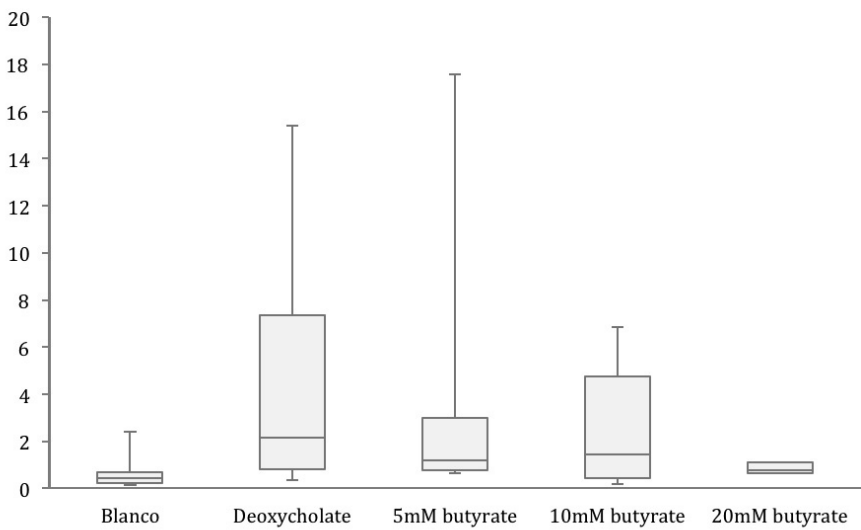

Figure 5.4 Sucralose flux at $\mathrm{t}=60$ minutes. Whisker plot showing median and quartiles.

\section{Discussion}

We have shown a beneficial effect of rectocolonic butyrate administration daily for one week on visceroperception in IBS patients. This observation extends on previous findings by our group in healthy volunteers ${ }^{16}$. The butyrate dosage we have employed (100 mM of sodium butyrate) is in the range of the maximum concentrations reached intraluminally, while taking a high-fiber diet. Therefore these doses can be considered as physiological ${ }^{24}$. The same dose of butyrate resulted in increased colonic levels of the antioxidant glutathione in healthy controls ${ }^{6}$, and also had clinical efficacy in the treatment of acute ulcerative colitis ${ }^{25,26}$.

The magnitude of the observed effect of butyrate was smaller in IBS patients, compared to that observed in healthy volunteers ${ }^{16}$. Several factors may account for this difference. First, IBS is a heterogeneous disorder, with variations in predominant 
symptoms, IBS subtypes and underlying pathophysiology. We have currently no indication or evidence that the effect of butyrate may differ among IBS sub-types or IBS patients with/without visceral hypersensitivity. The limited number of IBS patients we studied does not allow further subgroup analysis.

The beneficial effect of rectal butyrate on visceral sensitivity may result from various factors. First, direct modulation of 5-hydroxytryptamine (5-HT, serotonin) release by enterochromaffin cells may be involved. Serotonin was found to be released in rat colon after luminal stimulation by a mixture of the SCFAs including butyrate at concentrations of 100-200 mM SCFA ${ }^{27}$. Intestinal serotonin may beneficially modulate visceral perception via activation of the $5 \mathrm{HT}_{3}$ and $5 \mathrm{HT}_{4}$ receptors ${ }^{28}$ and perception thresholds may increase after serotonin release ${ }^{29}$. Butyrate, as well as other SCFAs, are able to trigger intestinal transient receptor potential vanniloid-1 (TRPV1) receptors. Prolonged TRPV1 stimulation may result in desensitisation of the TRPV1 receptor, thereby reducing the TRPV1 signalling to the central nervous system, and thus reducing pain sensations. Additionally, TRPV1 activation may initiate mucosal 5 -HT release in the gut and, consequently, alter visceral perception ${ }^{17}$.

Second, the effect of butyrate in modulating visceral perception may occur via its inhibitory effect on histone deacetylase. Several histone deacetylase inhibitors, including butyrate, have been reported to reduce inflammation-induced neurotoxicity in rats ${ }^{30}$. Valproate, another SCFA with histone deacetylase inhibitory capacity, is also able to potentiate the inhibitory transmitter gamma amino butyric acid (GABA). Furthermore, valproate improves neuropathic pain perception in type 2 diabetic patients $^{31}$. Butyrate may have a similar action on GABA, although human data available to support this hypothesis are lacking. Third, Haschke et al. described a direct effect of butyrate on isolated neurones. Butyrate increases intracellular $\mathrm{Ca}^{2+}$ levels, resulting in hyperpolarisation and, consequently, in an increase of the threshold required to evoke an action potential $\left.\right|^{32}$. This hyperpolarisation may lead to an increased adaptation to mechanical distension.

Fourth, the beneficial effect of butyrate on perception may occur via changes in mucosal inflammation. Persisting, ongoing low grade inflammation is a key factor in the pathogenesis of $\mathrm{IBS}^{33}$ and is associated with a pro-inflammatory genetic make up. We have shown previously in a study applying genome-wide micro-arrays, that butyrate induces the transcription of several genes that are involved in the coordination of anti-inflammatory and anti-oxidant status in intestinal mucosa ${ }^{6,34}$. Furthermore, we have demonstrated previously in an in vitro system using Caco2 cell lines, that butyrate reduces the release of several pro-inflammatory cytokines, improved transepithelial resistance and increased the concentration of the antioxidant glutathione ${ }^{35}$. This further supports the hypothesis that the beneficial effects of butyrate may (in part) result from its effect on mucosal inflammation.

The effects of butyrate on visceroperception that have been observed in animal models are not in line with data from human experiments. Butyrate instilled in the 
colon was reported to increase visceral perception and to maintain this increased visceral sensitivity in a rat model of TNBS-induced colitis ${ }^{36,37}$. These contrasting results of butyrate on visceral sensitivity between species may result from several factors. First, differences in the expression and activity of pain- or serotonin receptors may be involved. Second, differences in methodology of measuring visceral perception (VAS scores in humans versus behavioral changes in rats) may account for at least part of the observed differences. Third, fear and anxiety, which were perceived in a different way by both species, may have affected the pain scores ${ }^{38}$. Fourth, Kannampalli et al. state that the effects of butyrate inducing visceral hypersensitivity in rats are modulated via involvement of peptidergic C-fibers, which express TRPV1 ion channels. Fifth, different concentrations of butyrate have been used in the intervention studies, intestinal microbiota composition may differ as well as species dependent metabolism of butyrate ${ }^{17}$. Recently, Marger et al. demonstrated that T-type calcium channels are expressed in colonic nociceptive primary afferent neurons and contribute to the exaggerated pain perception in a butyrate-mediated rodent model of IBS ${ }^{39}$. Finally, butyrate has a direct effect on gut integrity by inducing re-organization and reallocation of tight junction proteins ${ }^{40}$.

Both inflammation and increased permeability are associated with IBS and IBS symptoms. The ex vivo experiment on colonic mucosal tissue strongly indicated a beneficial effect of butyrate on intestinal barrier function in a dose-dependent fashion. However, due to the limited number of experiments, in combination with the observed variation between individuals, statistical power was lacking and differences were not statistically significant. Nevertheless, our explorative data support previous findings that butyrate may have a potential beneficial effect in IBS via its ability to decrease colonic permeability ${ }^{10}$.

In general, our data confirm previous observations with respect to beneficial effects of butyrate treatment. We assume that dietary interventions with prebiotics leading to increased intracolonic butyrate concentrations may provide a nutritional strategy to influence visceroperception and thereby reduce IBS associated GI symptoms. Indeed, dietary interventions with prebiotics in healthy subjects were shown to result in an increased number of bifido bacteria, microbial fermentation rate and consequently in increased concentrations of SCFA ${ }^{41}$. Dietary intervention studies with non-digestible carbohydrates in IBS patients indicate that colonic fermentation of these carbohydrates may have detrimental effects on symptom severity in IBS patients, due to formation of byproducts of fermentation, such as hydrogen- and methane gas. A diet low in fermentable oligosaccharides, disaccharides, monosaccharides and polyols (FODMAP) was shown to decrease symptom severity for pain and bloating in IBS patients $^{42,43}$. It is currently not known whether dietary supplementation of butyrate producing fibers will result in symptom improvement, based on the beneficial effects of butyrate, or may also have undesirable effects due to increased gas production. 
In conclusion, we have shown that repeated rectocolonic butyrate administration for one week significantly reduces urge and pain sensations in IBS patients during mechanical rectal distensions. The consumption of non-digestible carbohydrates, which results in the production of butyrate in the colon due to microbial fermentation, may provide an attractive alternative dietary strategy to reduce visceral hypersensitivity and symptoms in IBS patients. 


\section{References}

1. Chang L. Review article: epidemiology and quality of life in functional gastrointestinal disorders. Aliment Pharmacol Ther 2004;20 Suppl 7:31-9.

2. Harkness EF, Harrington V, Hinder S, et al. GP perspectives of irritable bowel syndrome--an accepted illness, but management deviates from guidelines: a qualitative study. BMC family practice 2013;14:92.

3. Ludidi S, Conchillo JM, Keszthelyi D, et al. Rectal hypersensitivity as hallmark for irritable bowel syndrome: defining the optimal cutoff. Neurogastroenterol Motil 2012;24:729-33, e345-6.

4. van der Veek PP, Van Rood YR, Masclee AA. Symptom severity but not psychopathology predicts visceral hypersensitivity in irritable bowel syndrome. Clin Gastroenterol Hepatol 2008;6:321-8.

5. Boren J, Lee WN, Bassilian S, et al. The stable isotope-based dynamic metabolic profile of butyrateinduced HT29 cell differentiation. J Biol Chem 2003;278:28395-402.

6. Hamer HM, Jonkers DM, Bast A, et al. Butyrate modulates oxidative stress in the colonic mucosa of healthy humans. Clinical nutrition (Edinburgh, Scotland) 2009;28:88-93.

7. Ogawa $\mathrm{H}$, Rafiee $\mathrm{P}$, Fisher PJ, et al. Butyrate modulates gene and protein expression in human intestinal endothelial cells. Biochemical and biophysical research communications 2003;309:512-9.

8. Hamer HM, Jonkers D, Venema K, et al. Review article: the role of butyrate on colonic function. Aliment Pharmacol Ther 2008;27:104-19.

9. Soldavini J, Kaunitz JD. Pathobiology and potential therapeutic value of intestinal short-chain fatty acids in gut inflammation and obesity. Dig Dis Sci 2013;58:2756-66..

10. Suzuki T, Yoshida S, Hara H. Physiological concentrations of short-chain fatty acids immediately suppress colonic epithelial permeability. Br J Nutr 2008;100:297-305.

11. Annahazi A, Ferrier L, Bezirard V, et al. Luminal cysteine-proteases degrade colonic tight junction structure and are responsible for abdominal pain in constipation-predominant IBS. Am J Gastroenterol 2013;108:1322-31.

12. Barbara G, Zecchi L, Barbaro R, et al. Mucosal permeability and immune activation as potential therapeutic targets of probiotics in irritable bowel syndrome. J Clin Gastroenterol 2012;46 Suppl: S52-5.

13. Piche $T$, Barbara $G$, Aubert $P$, et al. Impaired intestinal barrier integrity in the colon of irritable bowel syndrome patients: involvement of soluble mediators. Gut 2009;58:196-201.

14. Vivinus-Nebot $M$, Frin-Mathy $G$, Bzioueche $H$, et al. Functional bowel symptoms in quiescent inflammatory bowel diseases: role of epithelial barrier disruption and low-grade inflammation. Gut 2013.

15. Kanauchi O, Mitsuyama K, Komiyama Y, et al. Preventive effects of enzyme-treated rice fiber in a restraint stress-induced irritable bowel syndrome model. International journal of molecular medicine 2010;25:547-55.

16. Vanhoutvin SA, Troost FJ, Kilkens TO, et al. The effects of butyrate enemas on visceral perception in healthy volunteers. Neurogastroenterol Motil 2009;21:952-e76.

17. Kannampalli P, Shaker R, Sengupta JN. Colonic butyrate- algesic or analgesic? Neurogastroenterol Motil 2011;23:975-9.

18. Hamer HM, Jonkers DM, Vanhoutvin SA, et al. Effect of butyrate enemas on inflammation and antioxidant status in the colonic mucosa of patients with ulcerative colitis in remission. Clinical nutrition (Edinburgh, Scotland) 2010;29:738-44.

19. Munch A, Strom M, Soderholm JD. Dihydroxy bile acids increase mucosal permeability and bacterial uptake in human colon biopsies. Scand J Gastroenterol 2007;42:1167-74.

20. Geraedts MC, Troost FJ, Tinnemans R, et al. Release of satiety hormones in response to specific dietary proteins is different between human and murine small intestinal mucosa. Annals of nutrition \& metabolism 2010;56:308-13.

21. van Wijck K, van Eijk HM, Buurman WA, et al. Novel analytical approach to a multi-sugar whole gut permeability assay. J Chromatogr B Analyt Technol Biomed Life Sci 2011;879:2794-801.

22. Lindsey PJ, Kaufmann J. Analysis of a longitudinal ordinal response clinical trial using dynamic models. Journal of the Royal Statistical Society Series C-Applied Statistics 2004;53:523-37. 
23. Lindsey JK. Statistical analysis of stochastic processes in time. Cambridge, UK; New York: Cambridge University Press 2004.

24. Cummings JH, Pomare EW, Branch WJ, et al. Short chain fatty acids in human large intestine, portal, hepatic and venous blood. Gut 1987;28:1221-7.

25. Luhrs H, Gerke T, Muller JG, et al. Butyrate inhibits NF-kappaB activation in lamina propria macrophages of patients with ulcerative colitis. Scand J Gastroenterol 2002;37:458-66.

26. Scheppach W, Sommer H, Kirchner T, et al. Effect of butyrate enemas on the colonic mucosa in distal ulcerative colitis. Gastroenterology 1992;103:51-6.

27. Fukumoto S, Tatewaki M, Yamada T, et al. Short-chain fatty acids stimulate colonic transit via intraluminal 5-HT release in rats. Am J Physiol Regul Integr Comp Physiol 2003;284:R1269-76.

28. Gershon MD, Liu MT. Serotonin and neuroprotection in functional bowel disorders. Neurogastroenterol Motil 2007;19 Suppl 2:19-24.

29. Greenwood-van Meerveld B. Importance of 5-hydroxytryptamine receptors on intestinal afferents in the regulation of visceral sensitivity. Neurogastroenterol Motil 2007;19 Suppl 2:13-8.

30. Chen PS, Wang CC, Bortner CD, et al. Valproic acid and other histone deacetylase inhibitors induce microglial apoptosis and attenuate lipopolysaccharide-induced dopaminergic neurotoxicity. Neuroscience 2007;149:203-12.

31. Kochar DK, Jain N, Agarwal RP, et al. Sodium valproate in the management of painful neuropathy in type 2 diabetes - a randomized placebo controlled study. Acta neurologica Scandinavica 2002;106:248-52.

32. Haschke G, Schafer H, Diener M. Effect of butyrate on membrane potential, ionic currents and intracellular $\mathrm{Ca} 2+$ concentration in cultured rat myenteric neurones. Neurogastroenterol Motil 2002;14:133-42.

33. Ford AC, Talley NJ. Mucosal inflammation as a potential etiological factor in irritable bowel syndrome: a systematic review. J Gastroenterol 2011;46:421-31.

34. Vanhoutvin SA, Troost FJ, Hamer HM, et al. Butyrate-induced transcriptional changes in human colonic mucosa. PLoS ONE 2009;4:e6759.

35. Hamer HM. Short chain fatty acids and colonic health; PhD thesis, Maastricht University 2009:49-69.

36. Bourdu S, Dapoigny $M$, Chapuy $E$, et al. Rectal instillation of butyrate provides a novel clinically relevant model of noninflammatory colonic hypersensitivity in rats. Gastroenterology 2005;128: 1996-2008.

37. Tarrerias AL, Millecamps M, Alloui A, et al. Short-chain fatty acid enemas fail to decrease colonic hypersensitivity and inflammation in TNBS-induced colonic inflammation in rats. Pain 2002;100:91-7.

38. Rhudy JL, Meagher MW. Fear and anxiety: divergent effects on human pain thresholds. Pain 2000;84:65-75.

39. Marger F, Gelot A, Alloui A, et al. T-type calcium channels contribute to colonic hypersensitivity in a rat model of irritable bowel syndrome. Proceedings of the National Academy of Sciences of the United States of America 2011;108:11268-73.

40. Ohata A, Usami M, Miyoshi M. Short-chain fatty acids alter tight junction permeability in intestinal monolayer cells via lipoxygenase activation. Nutrition 2005;21:838-47.

41. Klosterbuer AS, Hullar MA, Li F, et al. Gastrointestinal effects of resistant starch, soluble maize fibre and pullulan in healthy adults. Br J Nutr 2013;110:1068-74.

42. Staudacher HM, Lomer MC, Anderson JL, et al. Fermentable carbohydrate restriction reduces luminal bifidobacteria and gastrointestinal symptoms in patients with irritable bowel syndrome. J Nutr 2012;142:1510-8.

43. Staudacher HM, Whelan K, Irving PM, et al. Comparison of symptom response following advice for a diet low in fermentable carbohydrates (FODMAPs) versus standard dietary advice in patients with irritable bowel syndrome. J Hum Nutr Diet 2011;24:487-95. 


\section{Extensive statistical information}

All data analyses take as much as possible of the experimental design into account as to provide the most appropriate analysis at the time of the writing of the manuscript. In addition, all analyses have been performed in a similar way in order to remain consistent throughout the manuscript.

\section{Visceral perception data analysis}

Exactly as described previously ${ }^{1}$, the pain and discomfort data were analysed using a multivariate Gaussian non-linear regression $(\operatorname{MVN}(\mu, \Sigma)$ where $\mu$ is the mean,

$\sum$ is the covariance matrix $\left(\begin{array}{cccc}\sigma^{2}+\delta & \delta+\rho^{t_{2}-t_{1}} & \cdots & \delta+\rho^{t_{n}-t_{1}} \\ \delta+\rho^{t_{2}-t_{1}} & \ddots & \ddots & \vdots \\ \vdots & \ddots & \ddots & \delta+\rho^{t_{n}-t_{n-1}} \\ \delta+\rho^{t_{n}-t_{1}} & \cdots & \delta+\rho^{t_{n}-t_{n-1}} & \sigma^{2}+\delta\end{array}\right), \sigma^{2}$ is

the variance, $\rho^{t_{n}-t_{n-1}}$ is the first order autocorrelation taking the time lag with the previous observation into account, and $\delta$ as both the extra component of variance across subjects and the common covariance among responses on the same subject) including, if necessary, a random effect and a first order autocorrelation.

The inference criterion used for comparing the models is their ability to predict the observed data, i.e. models are compared directly through their minimised minus loglikelihood. When the numbers of parameters in models differed, they were penalised by adding the number of estimated parameters, a form of the Akaike information criterion $(\mathrm{AIC})^{2}$.

For each variable of interest, a model containing the relevant covariates $\left(\mathrm{E}(y)=\beta_{0}+\beta_{1} \times\right.$ Pressure $+\beta_{2} \times \mathrm{MDP}+\beta_{3} \times \mathrm{FS}+\beta_{4} \times$ Procedure $+\beta_{5} \times$ Carry-Over ) was fitted in order to obtain a reference AIC. Then a model containing the dose was fitted $\left(\mathrm{E}(y)=\beta_{0}+\beta_{1} \times\right.$ Press. $+\beta_{2} \times \mathrm{MDP}+\beta_{3} \times \mathrm{FS}+\beta_{4} \times$ Proc.$+\beta_{5} \times$ Carry-Over $+\beta_{6} \times$ Dose $)$.

The variable of interest under consideration was found to be differentially expressed if the AIC of the model containing the dose effect was smaller when compared to the reference AIC (the model not containing the dose effect).

Urge was scored on an ordinal 6-point scale and was analysed using a mixture of a logistic distribution (parameterised as a proportional-odds) and a gamma distribution (to introduce frailty and autocorrelation dependencies) ${ }^{3}$.

The first model was obtained by imposing the mean regression to follow a logistic ('S-shape') curve through the pressure variable $\left(\beta_{0} /\left(1+e^{\left(\beta_{1}+\beta_{2} \times \text { Pressure }\right)}\right)\right)$. The model included pressure, MDP, first sensation, intervention (placebo or butyrate), carry over effect, and the HADS scores as explanatory variables. The AIC was used to assess whether there was a dose effect. 


\section{Abdominal symptoms and stool consistency analysis}

Similarly to urge, the cramping, gas, and bloating complaints as well as the stool type were analysed using a mixture of a logistic distribution (parameterized as a proportional-odds) and a gamma distribution (to introduce frailty and autocorrelation dependencies) ${ }^{3}$. The model included time, carry over effect, intervention (placebo or butyrate), and the HADS scores (except for stool type) as explanatory variables. As previously, the AIC was used to assess whether there was an intervention effect.

\section{Stool frequency analysis}

Fecal frequency is a count variable and was analysed using a mixture of gamma distributions (to introduce a serial dependence). A reference AIC was obtained by using a linear mean regression $\left(\beta_{0}+\beta_{1} \times\right.$ Day $)$. The model included time, carry over effect, and procedure (treated or not) as explanatory variables. The AIC was used to assess whether there was an intervention effect.

\section{Compliance data analysis}

Intestinal compliance data were analysed using a multivariate Gaussian non-linear regression ( $\operatorname{MVN}(\mu, \Sigma)$ where $\mu$ is the mean, $\Sigma$ is the covariance matrix

$$
\left(\begin{array}{cccccc}
\sigma^{2}+\tau_{1}+\tau_{2} & \cdots & \tau_{1}+\tau_{2} & \tau_{1} & \cdots & \tau_{1} \\
\vdots & \ddots & \vdots & \vdots & \ddots & \vdots \\
\tau_{1}+\tau_{2} & \cdots & \sigma^{2}+\tau_{1}+\tau_{2} & \tau_{1} & \cdots & \tau_{1} \\
\tau_{1} & \cdots & \tau_{1} & \sigma^{2}+\tau_{1}+\tau_{2} & \cdots & \tau_{1}+\tau_{2} \\
\vdots & \ddots & \vdots & \vdots & \ddots & \vdots \\
\tau_{1} & \cdots & \tau_{1} & \tau_{1}+\tau_{2} & \cdots & \sigma^{2}+\tau_{1}+\tau_{2}
\end{array}\right), \sigma^{2} \text { is the variance, } \tau_{1}
$$

gives the relationship among observations measured every 30 seconds on the same day, whereas $\tau_{2}$ gives the relationship between days) and including, if necessary, two random effects. As for the analysis of pain and discomfort, a model containing the intervention effect was fitted. The model included time, intervention (placebo or butyrate), carry over effect, MDP, and first sensation as explanatory variables. The AIC was then used to assess if the intervention effect was significant. 


\section{References}

1. Vanhoutvin SA, Troost FJ, Kilkens TO, Lindsey PJ, Hamer HM, Jonkers DM, et al. The effects of butyrate enemas on visceral perception in healthy volunteers. Neurogastroenterol Motil. 2009 Sep;21(9):952-e76.

2. Akaike H. Information theory and an extension of the maximum likelihood principle. Second international symposium on inference theory (Tsahkadsor, 1971). 1973:267-81.

3. Lindsey PJ, Kaufmann J. Analysis of a longitudinal ordinal response clinical trial using dynamic models. Journal of the Royal Statistical Society Series C-Applied Statistics. 2004;53:523-37. 


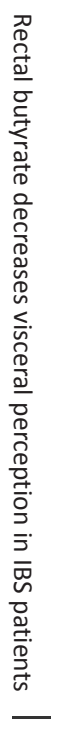



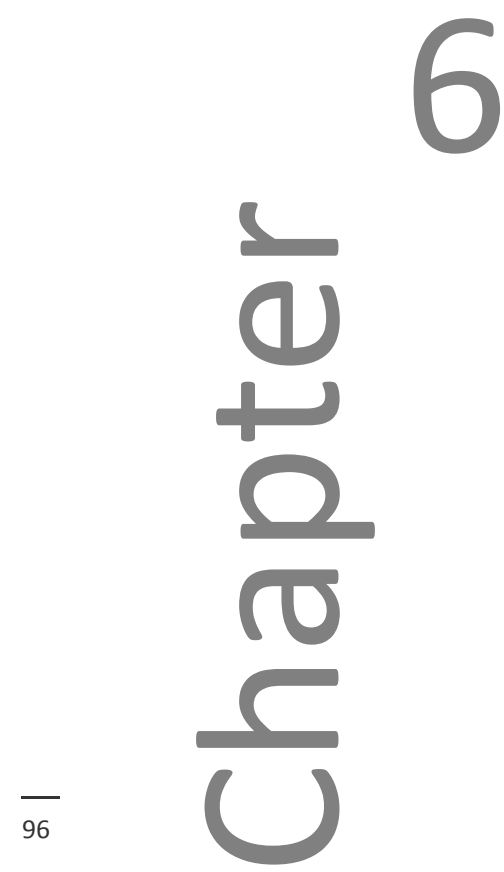


\section{Alternative procedure to shorten rectal barostat procedure for the assessment of rectal compliance and visceral perception: a feasibility study}

Steven ALW Vanhoutvin, Freddy J Troost, Tessa OC Kilkens, Patrick J Lindsey, Daisy MAE Jonkers, Koen Venema, Ad AM Masclee, Robert JM Brummer, J Gastroenterol. 2012;47:896-903. 


\section{Abstract}

\section{Background}

Barostat methodology is widely used for assessing visceral perception. Different barostat protocols are described with respect to the measurement of rectal compliance and visceral perception. The choice of protocols affects the duration, which is normally 60-90 minutes, and accuracy of the procedure. This study aimed to shorten the procedure by using the semirandom distension protocol for both compliance and visceral perception measurement and a correction based on rectal capacity (RC) instead of minimal distension pressure (MDP).

\section{Methods}

Twelve Irritable Bowel Syndrome (IBS) patients (7 females) and eleven healthy controls (8 females) underwent a barostat procedure. Compliance was determined during both a staircase distension and a semi-random protocol. Visceral perception data were compared as a function of pressure or relative volume, corrected for MDP or RC, respectively.

\section{Results}

Compliance measurement using the semi-random protocol instead of the staircase distension protocol resulted in an over-estimation in healthy volunteers, but not in IBS patients. The overall conclusion that IBS patients had a lower compliance compared to controls was not different between protocols. Data presentation of the visceral perception scores as function of corrected volume instead of pressures corrected for MDP, did not alter the conclusion that sensation scores in IBS patients were higher as compared to healthy controls.

\section{Conclusions}

This study showed that barostat procedures may be shortened by approximately 20 minutes, without losing the ability to discriminate between healthy controls and IBS patients. Complementary, a correction for RC instead of MDP may improve the accuracy of the procedure. 


\section{Introduction}

Alterations in visceral perception and rectal compliance have been observed in several functional gastrointestinal disorders, but the underlying pathophysiological mechanisms are still poorly understood. Several studies demonstrated a decreased rectal compliance and increased rectal sensitivity, in patients with Irritable Bowel Syndrome (IBS), compared to healthy controls ${ }^{1-7}$. Visceral perception is generally measured in vivo using the barostat technique. Since its introduction, different distension protocols have been used and efforts have been made to optimise the distension protocols ${ }^{8-10}$. Whitehead et al. described a number of basic recommenddations for the measurement of visceral perception and compliance. These recommendations include the use of a thin plastic polyethylene bag instead of a latex balloon, inflation speed, catheter construction in terms of minimal luminal cross sections and pressure monitoring inside the balloon, the use of Visual Analogue Scales (VAS) and the influence of body posture and position during the measurements ${ }^{11}$. In addition, recommendations were given with respect to the distension protocol for determination of compliance, visceral perception, determination of minimal distension pressure (MDP) and first sensation. However, barostat procedures applied for clinical diagnostic purposes and for scientific studies still have different protocols. This hampers comparisons between studies. Some but not all research groups present sensation scores (pain, urge and discomfort) as a function of balloon pressure ${ }^{12-15}$, while others relate it to balloon volume ${ }^{9,14}$. In order to correct for inter-individual variation, a correction for MDP and/or rectal capacity (RC) is used by some, but not by others. Moreover, the protocols used to determine MDP and RC differ.

To enable the comparison of results obtained in different studies, initiatives should be taken to come to a generally accepted protocol with standardised cut-offs for RC and/or MDP. Consensus should be achieved with respect to the pressure, at which rectal capacity should be determined. MDP is defined by some investigators, as the pressure at which respiratory waves appear for the first time in the volume curve ${ }^{9,13}$, while others define it as the pressure needed to reach a specific volume (e.g. pressure at which the volume reaches $25 \mathrm{ml})^{4,10}$. Determination of the different parameters in one barostat procedure requires multiple consecutive distension protocols. Shortening the procedure by combining the determination of several parameters in one distension protocol would provide a major advantage for its use in a clinical setting, since duration of the procedure has important implications for patient burden as well as for total costs.

The primary aim of this study was to shorten the barostat procedure by using the semi-random distension protocol for both compliance and visceral perception measurement, while preserving the ability to discriminate between healthy volunteers and IBS patients. This would shorten the duration of the barostat protocol and, hence, lower the patient- and labour burden. 


\section{Methods}

\section{Subjects}

Twelve IBS patients, based on Rome III criteria ( 7 females, mean age $42 \pm 14$ years) and eleven healthy controls ( 8 females, mean age $33 \pm 15$ years) were included in this study. Five of the IBS patients had diarrhea-predominant IBS, 5 suffered from constipation-predominant IBS, and 2 patients had the alternating type. No differences were found based on age or gender between both groups. BMI $\left(\mathrm{kg} / \mathrm{m}^{2}\right)$ did not significantly differ between IBS patients (mean 24; $\mathrm{Cl}$ 22.5-25.5) and healthy volunteers (mean 23.9; $\mathrm{Cl}$ 22.1-25.8). None of the volunteers had a history of abdominal surgery. No medication was allowed during the study unless subjects were on stable medication for at least three months prior to and during the study. The study was approved by the Medical Ethics Committee of University Hospital Maastricht and conducted in accordance with the principles of the 'Declaration of Helsinki' (52nd WMA General Assembly, Edinburgh, Scotland, Oct 2000). All volunteers gave their written informed consent prior to participation. Baseline data from two intervention studies (http://www.clinicaltrials.gov, NCT00696098 and NCT00726817) were used for the present study. All subjects participated in a single barostat measurement.

\section{Barostat protocol}

All subjects underwent the same barostat procedure as described before ${ }^{13}$. After an overnight fast, the subjects arrived in the hospital and self-administered a rectal enema containing $60 \mathrm{ml}$ of saline to clean the rectum. Five minutes thereafter, patients were instructed to void rectal contents.

Subsequently, the patients laid down on a bed in a left lateral supine position and remained in this position during the entire test procedure. This position was chosen to minimize the intra-abdominal pressure. A commercially available barostat balloon (Mui Scientific C7-2CB-R, Ontario, Canada) was lubricated with KY gel (Johnsson \& Johnsson, Langhorne, Pennsylvania) and inserted rectally $3 \mathrm{~cm}$ proximal to the anal sphincter. The balloon had a volume of $500 \mathrm{ml}$ and was made of PVC. After a 5 minutes habituation period, the balloon was attached to the barostat equipment (Distender II, G\&J electronics, Ontario, Canada) and the barostat procedure was started. The controlled balloon distensions were programmed using the standard software package of the barostat equipment (Protocol Plus Deluxe, version 6_7; G\&J electronics, Ontario, Canada).

The barostat protocol consisted of five sub-protocols, each designed for the measurement of specific parameters of interest (Figure 6.1). 


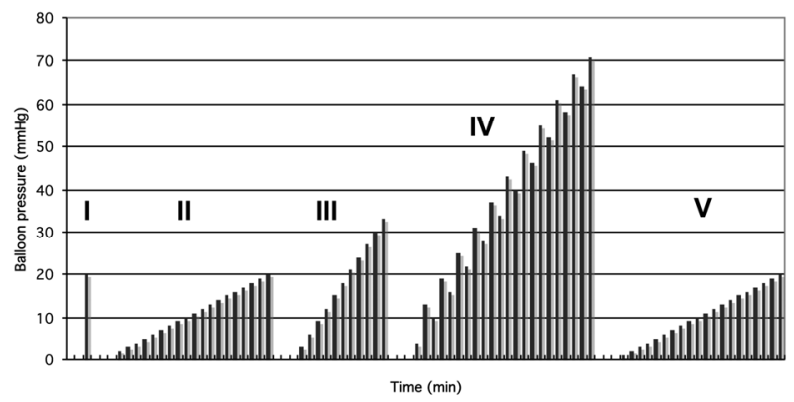

Figure 6.1 Barostat protocol that was applied in this study. It contained 5 consecutive distension protocols (I-V). Protocol I was designed for balloon unfolding, protocol II for determination of minimal distension pressure (MDP-1) and first sensation (FS-1), protocol III for compliance 1 and rectal capacity (RC) measurement, protocol IV for visceral perception and compliance 2 and protocol $\mathrm{V}$ for the assessment of MDP-2 and FS-2.

The total duration of the barostat procedure was 60-90 minutes. After inclusion, prior to the start of the study, all subjects underwent a dummy barostat procedure, which consisted of a reduced number of distensions of different intensities. During this dummy barostat procedure, subjects were to get familiar with the barostat technique and the VAS scores in order to reduce the amount of fear and anxiety on the day of testing.

\section{I) Balloon unfolding}

The first part of the protocol consisted of a single distension at a balloon pressure of $20 \mathrm{mmHg}$ for 1 minute, to ensure that the balloon was placed correctly without folds that may impair airflow.

\section{Il and V) Minimal Distension Pressure (MDP)}

The second part of the protocol consisted of a staircase distension protocol with pressure steps of $1 \mathrm{mmHg}$ with a duration of 30 seconds each and a range from 0 $20 \mathrm{mmHg}$. The MDP, which is the minimal balloon pressure required to overcome the intra-abdominal pressure, was defined as the first pressure at which respiratory curves were present in the volume recording of the balloon. The entire protocol was performed up to the $20 \mathrm{mmHg}$ pressure in all subjects and served as a sensitisation step prior to the compliance and perception measurements. The obtained MDP value was set to zero as a reference point during the measurement of visceral perception (protocol IV). During this protocol the patients were asked to report the moment at which they sensed the balloon for the first time. This pressure was defined as the threshold for first sensation (FS). The measurement of MDP and FS were repeated at 
the end of the protocol (protocol V) to check the stability of these variables during the barostat procedure.

\section{III) Compliance and Rectal Capacity}

Directly after finishing the MDP and FS measurements, the third part of the protocol was initiated. This part of the protocol, designed for determining compliance, consisted of a staircase distension protocol with pressure steps of $3 \mathrm{mmHg}$ with a duration of 30 seconds each and a pressure range of 0-33 $\mathrm{mmHg}$. Pressure-volume curves from both the staircase distension (part III of the protocol, i.e. compliance 1) and the semi-random distension (part IV of the protocol, i.e. compliance 2) were used to compare the compliance measurements. Dynamic compliance was defined as the slope of the pressure-volume curve at the steepest part (at the inflection point of the curve). In addition, rectal capacity, which was defined as the volume at a pressure of $33 \mathrm{mmHg}$, was determined. RC was used to correct the measured volumes for differences in individual RC. Consequently, all volumes are expressed as a percentage of the individual RC (= index volume).

\section{IV) Visceral perception}

Subsequently, the distension protocol of the visceral perception measurements was initiated. This protocol consisted of semi-random distensions (at 4, 13, 10, 19, 16, 25, $22,31,28,37,34,43,40,49,46,55,52,61,58,67,64,71 \mathrm{mmHg}$ above MDP, respectively) with a duration of 1 minute each, interspaced with 30 second intervals at MDP. Thirty seconds after the start of each distension, patients scored the sensation of pain and discomfort on a $10 \mathrm{~cm}$ Visual Analogue Scale (VAS) and urge on a 6-point scale $(0=$ no feeling, $1=$ just sensible , $2=$ clearly sensible/ light urge, $3=$ normal urge, $4=$ strong urge/ have to run to toilet, $5=$ maximum/stop) represented by 6 buttons on an electronic control panel (distender II perception panel), which was directly linked to the barostat equipment. The procedure was stopped when the maximum score for pain, urge or discomfort was reached.

\section{Statistical analysis}

\section{Minimal distension pressure (MDP) and first sensation (FS) data analysis}

MDP and FS were each analysed using a Gaussian linear regression. For both analyses, the body mass index (BMI), FS, and compliance were included during model building. The inference criterion used for comparing the models is their ability to predict the observed data, i.e. models are compared directly through their minimized minus loglikelihood. When the numbers of parameters in models differed, they were penalized by adding the number of estimated parameters, a form of the Akaike information criterion $(\mathrm{AIC})^{16}$. For each variable of interest, the group was then added to the model. 
The effects were considered significant if the AIC decreased compared to the model not containing the group.

MDP, BMI, and rectal capacity (RC) were also analysed by pairs using a bivariate Gaussian linear regression including the appropriate covariance structure in order to capture the dependence between them. The compliance and FS were included as explanatory variables during model building. The AIC was used to assess whether there was a group effect.

\section{Rectal capacity data analysis}

The RC volume was analyzed using a Gaussian non-linear regression including the pressure and compliance as explanatory variables. The AIC was used to assess whether there was a group effect.

\section{Visceral perception data analysis}

The pain and discomfort data were analyzed using a multivariate Gaussian non-linear regression including, if necessary, a random effect and a first order autocorrelation. Urge was scored on an ordinal 6-point scale and was analyzed using a mixture of a logistic distribution (parameterized as a proportional-odds) and a gamma distribution (to introduce frailty and autocorrelation dependencies) ${ }^{17}$. The mean regression was imposed through the pressure variable to follow a logistic ('S-shape') curve. The model included MDP and FS as explanatory variables. As for the other analysis, the AIC was used to assess whether there was a group effect.

A more detailed description of the analyses is provided in the supplementary material (S6.1).

\section{Results}

\section{Visceral perception}

Figures 6.2A-2F show the perception scores for pain, urge and discomfort presented as a function of pressure (corrected for individual differences in MDP) and as a function of index volume (corrected for individual differences in RC). As shown in Figure $6.2 \mathrm{~A}$, the index volume at which a moderate pain level of $50 \%$ is reached is 1.11 and 1.24 for IBS patients and healthy controls, respectively. The confidence intervals for the pains cores at the level of index volume are 44.15-54.97 and 42.34-54.32 for IBS patients and healthy controls, respectively. The individual curves for the two conditions differ significantly. In all cases, IBS patients showed higher sensation scores compared to the healthy controls independent of the presentation of pressure or index volume curves. 
2A) Pain versus Index Volume

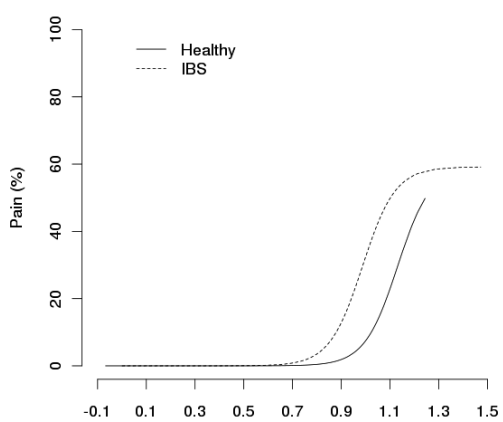

2C) Discomfort versus Index Volume

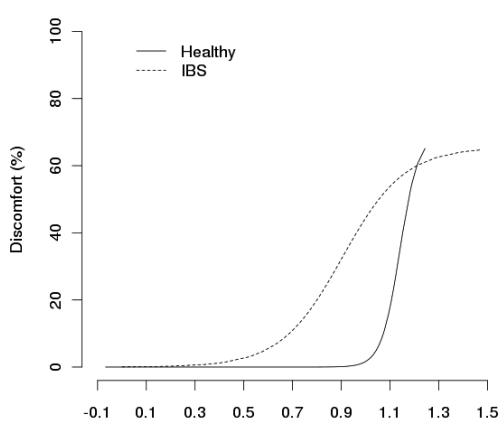

2E) Urge versus Index Volume

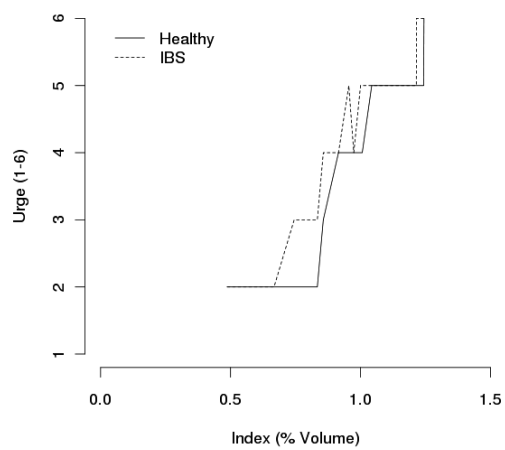

2B) Pain versus Pressure

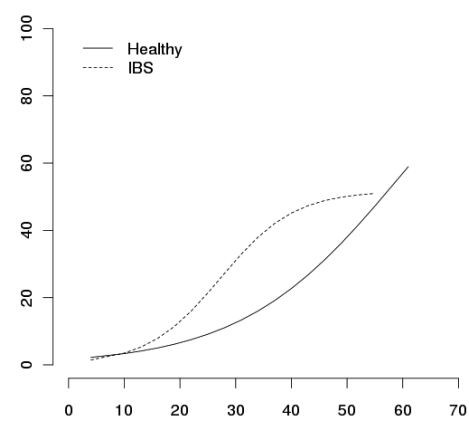

2D) Discomfort versus Pressure

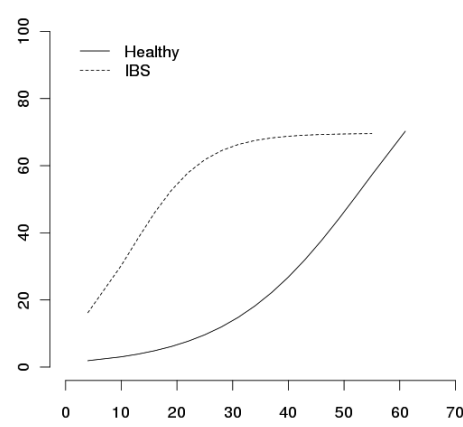

2F) Urge versus Pressure

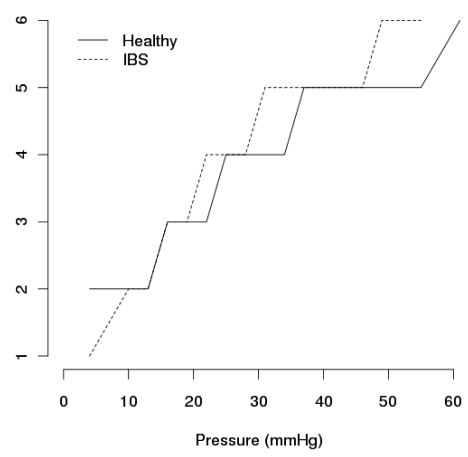

Figure 6.2 The perception scores for pain, discomfort and urge presented as either a function of index volume (Figures $6.2 \mathrm{~A}, 6.2 \mathrm{C}$ and $6.2 \mathrm{E}$, respectively) or as function of pressure (Figures 6.2B, $6.2 \mathrm{D}$ and $6.2 \mathrm{~F}$, respectively) in IBS patients and in healthy controls. In all cases, IBS patients showed higher sensation scores compared to healthy controls. 


\section{MDP and FS}

MDP and FS were determined in the beginning (1) and at the end (2) of the protocol. No significant differences were detected between MDP1 (mean 4.9; $\mathrm{Cl} 4.1-5.7$ ) and MDP2 (mean 5.3; $\mathrm{Cl} 4.6-6.1$ ) and between FS1 (mean 12.1; $\mathrm{Cl} 10.5-13.7$ ) and FS2 (mean 11.9; $\mathrm{Cl}$ 10.3-13.5).

IBS patients had a lower FS as compared to healthy controls (mean $6.81 \mathrm{mmHg}$; $\mathrm{Cl}=5.14-8.74$ and mean $12 \mathrm{mmHg} ; \mathrm{Cl}=10.73-13.63$, respectively). No significant correlation was found between MDP and RC. (Figure 6.3) The correlation as was shown in Figure 6.3, was RC=337.4-9.6*MDP with a confidence interval of the coefficient of -20.2 to 1.0 indicating that the correlation is not significant. BMI and $\mathrm{MDP}$, and between BMI and RC (data not shown).

Rectal Capacity versus MDP

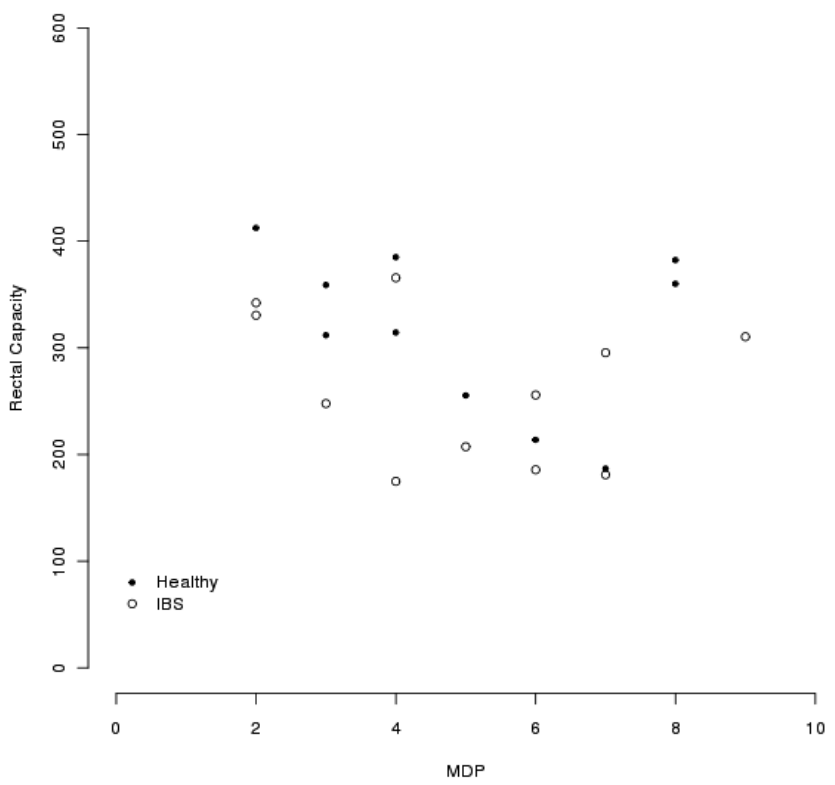

Figure 6.3 Shows the individual measurements (two missing values) of Rectal Capacity (RC) and Minimal Distension Pressure (MDP). No significant correlation was found.

\section{$\mathrm{RC}$}

$\mathrm{RC}$ was determined as the volume at a pressure of $33 \mathrm{mmHg}$ and was used to plot the sensation scores as a function of index volume (volume \% of individual RC). RC was not significantly different between healthy volunteers (mean $1.1 ; \mathrm{Cl} 1.0-1.2$ ) and IBS patients (mean 1.1; Cl 1.1-1.2). 


\section{Compliance}

Within IBS patients, no difference was found between the compliance calculated in part III and IV of the protocol (compliance 1 and 2, respectively). In healthy controls, calculation of the compliance in the semi-random protocol (compliance 2 ) resulted in a higher compliance (Figure 6.4). Regardless of the protocol chosen, the compliance was significantly lower in IBS patients compared to healthy controls. In addition to a comparison of the overall pressure-volume curves, dynamic compliance was calculated at the inflection point of the pressure-volume curves from Figure 6.4. The means and confidence intervals for the dynamic compliance 1 and 2 for the healthy controls were $156.86 \mathrm{ml} / \mathrm{mmHg} ; \mathrm{Cl}=155.6-158.12$ and $199.89 \mathrm{ml} / \mathrm{mmHg}$; $\mathrm{Cl}=198.75-201.04$, respectively and for the IBS patients $133.3 \mathrm{ml} / \mathrm{mmHg} ; \mathrm{Cl}=132.06$ 134.54 and $137.81 \mathrm{ml} / \mathrm{mmHg} ; \mathrm{Cl}=136.56-139.05$, respectively.

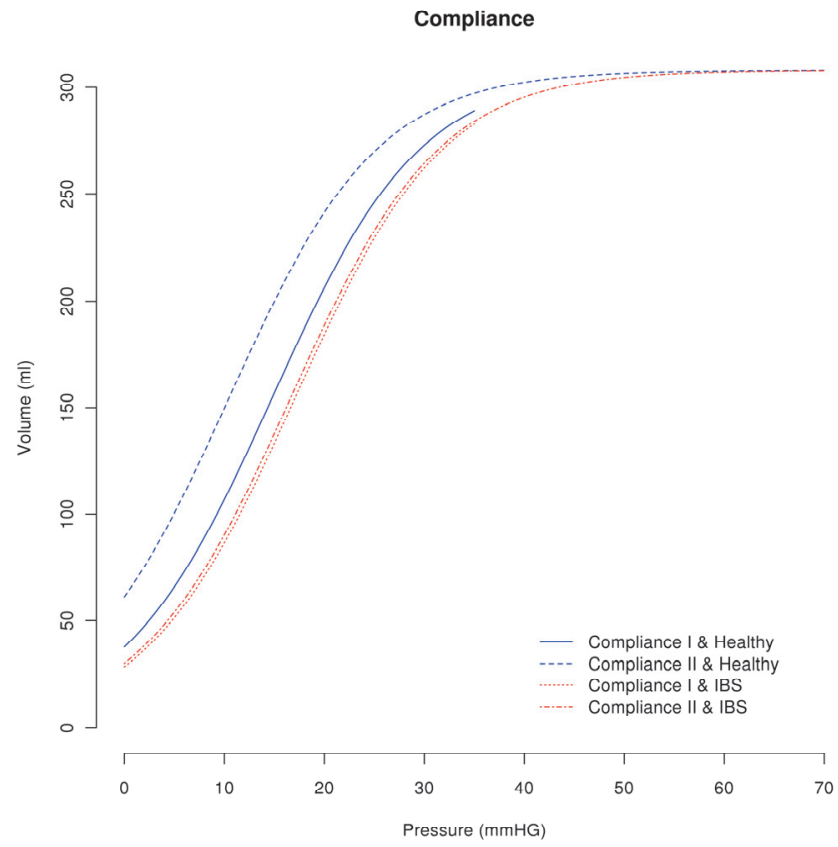

Figure 6.4 Compliance curves for healthy controls and IBS patients, both calculated in the staircase distension protocol (compliance 1) and in the semi-random protocol ( compliance 2).

\section{Discussion}

Our data indicate that compliance can be measured in the semi-random protocol instead of the staircase distension protocol, without losing the ability to discriminate between healthy controls and IBS patients. Furthermore, measurements of MDP and 
FS did not change during the barostat procedure. The visceral perception data, expressed as percentage of RC show the same results as those based on balloon pressure, although the presentation of the data differs. Both sets of data lead to the conclusion that perception scores are higher in IBS patients compared to controls. Baseline data from two intervention studies using the same procedure were used for the present study. This has led to two highly comparable datasets but also resulted in a lack of perception data in the staircase distension protocol for evaluation of the possibility of measuring multiple parameters in the staircase protocol. The number of patients tested for this study did not allow sub-group analysis of different types of IBS patients.

In literature, various methods are applied to determine rectal compliance from a pressure-volume curve ${ }^{8-10}$. Both the total fit and the dynamic compliance, which is the slope of the pressure-volume curve at its steepest point, are commonly used techniques to evaluate the compliance. The total fit of the curve provides more information on the pressure-volume relationship at each pressure level without losing statistical power due to multiple testing. In the present study, a total fit of the curve was calculated to evaluate the differences between IBS patients and healthy controls using two different distension protocols (i.e. semi-random vs. staircase). In addition, the compliance values at the steepest point of the pressure-volume curve (dynamic comliance) was presented. With the interpretation of his dynamic compliance, however, several factors in the protocol should be taken into account that may have influenced the result and therefore stress a proper comparison between studies (balloonshape and -characteristics, pressure- vs. volume-controlled distensions and the size of pressure or volume increments in the protocol).

In IBS, no difference was detected in compliance measured using the two distension protocols (compliance I and II), indicating that compliance can be measured in the semi-random protocol used to assess visceral perception. In healthy volunteers however, compliance measured in the semi-random protocol resulted in higher values compared to the values calculated in the staircase distension. The reason for this difference may result from the fact that healthy controls have a higher rectal compliance. The barostat device is designed to inflate or extract air from the balloon in order to maintain a certain pressure. During the semi-random staircase distension, the barostat device deflates the balloon after each pressure step, until the pressure in the balloon equals MDP. The volume at which this pressure is reached, depends on the rectal tone and probably on intra-abdominal pressure. Unfortunately, we were unable to find evidence for the latter since no correlation was found between MDP and compliance. Another possible explanation for the higher volumes measured in the semi-random protocol may be that the previous distension steps from the staircase distension led to rectal adaptation and subsequent relaxation. Nozu et al. reported a sensitizing effect of priming distensions in IBS patients whereas no effect of priming on sensitivity was observed in healthy volunteers ${ }^{18}$. This suggests that a difference in adaptation between healthy volunteers and IBS patients exists. We showed that 
compliance measurement in the semi-random protocol increases the difference between IBS patients and healthy controls and thus, will help to better discriminate between those groups. An important implication of this observation is, that the conventional staircase distension for measuring compliance can be discarded from barostat protocols, which results in a reduction of the duration of the total procedure by approximately 10 minutes per patient.

The compliance measurement is mainly used for evaluation of the pathophysiology of gastrointestinal conditions ${ }^{5}$. Our results show that in addition to visceral perception, compliance may also be a useful diagnostic tool, and is able to discriminate between healthy controls and IBS patients.

MDP has been used in a large number of studies to correct for differences in intraabdominal pressure between subjects ${ }^{4,5,10,13,14,19,20}$. The variation that exists between the methods to determine MDP, hampers the comparison between various studies. Sometimes, MDP is reported as the pressure value at which the volume reaches $25 \mathrm{ml}$, while we and others defined MDP as the pressure at which respiratory waves could be detected in the balloon volume. In our opinion this method is more precise, as it allows the determination of MDP, independent of anatomical differences in the rectal capacity of the patients, although the possibility of substantial inter-observer variation should be considered when comparing different studies. In addition, the body position of the patient during MDP measurement should be considered carefully, since this greatly influences MDP. In this study, the patients were in a left lateral position to minimize the intra-abdominal pressure.

The MDP, as determined in the staircase distension, is used to correct for differences in abdominal pressure. This pressure is set to zero in the protocol for the measurement of visceral perception. A disadvantage of using the MDP as a reference is that it needs to be assessed, as well as programmed, during the actual measurement. The determination of MDP has a high inter-observer variability, which affects the accuracy of the further procedure. If MDP is set during the compliance measurement (instead of the semi-random protocol), information on the start of the pressure-volume curve will be lost since the curve will start at MDP instead of $0 \mathrm{mmHg}$. Hence, the use of MDP as a reference for barostat measurements makes the barostat technique prone to errors in conducting the measurements.

An alternative for the MDP correction could be a correction for RC. Where MDP is the balloon pressure needed to overcome the intra-abdominal pressure, RC is mostly defined as the volume at a certain pressure at the high end of the pressure range. A correlation between MDP and RC was not found. This suggests that RC, which is determined in the higher pressure range of the protocol was influenced by other factors (such as anatomical size of the rectum or stretch of non-contractile tissue) than MDP, which is known to be affected by differences in body posture and body position.

Fox et al. studied the minimal pressure at which RC should be determined with a minimal variance in the outcome measure ${ }^{9}$. They showed, based on results in healthy 
subjects that the variance of the RC determination decreased with increasing pressure and RC should be determined preferably at a pressure of $40 \mathrm{mmHg}$. In line with these findings but limited by the maximum range of our staircase protocol, we defined a pressure of $33 \mathrm{mmHg}$ to determine $\mathrm{RC}^{9}$. Although in the present study all IBS patients reached the pressure of $33 \mathrm{mmHg}$, the decreased pain threshold of IBS patients could potentially compromise a proper measurement of RC at higher pressure, because some patients may not complete the barostat protocol until this pressure is reached. We used a barostat balloon with a volume of $500 \mathrm{ml}$, whereas Fox et al. applied a larger balloon with a volume of $800 \mathrm{ml}$. This may have affected the pressure volume curves due to a difference in wall tension, hampering a comparison of both studies. Within the present study though, these effects are expected to be small since none of the_subjects reached the maximal balloon volume in the measurement of RC and all volunteers were measured by an identical protocol and equipment. The impact of both variables (balloon volume and pressure for RC measurement) should be studied in_detail in future validation studies to reach consensus on a fully standardized procedure. Based on previous findings that a semi-random protocol reduces the bias that is introduced by both the predictability of the protocol and differences in tendency to report pain ${ }^{21}$, we expect the ascending method of limits to give lower values for pain thresholds compared to phasic distensions in a random order. Conversely, Nozu et al. showed that phasic distensions may sensitize IBS patients, which may result in lower pain thresholds in a semi-random protocol ${ }^{18}$. We expect this sensitizing effect of the phasic distensions to be minimal in the lower volume/pressure range of the protocol since this is the first part of the assessment and only few distensions are needed to reach the value for first pain sensations. It should be noted that all subjects underwent a dummy barostat procedure after inclusion in the study, to reduce the amount of fear and anxiety on the actual day of testing and to prevent a learning curve in the consecutive test days, which may affect the study outcome.

The major advantage of correcting visceral perception data for RC, instead of MDP, is that RC correction can be done after the barostat procedure and does not require a dedicated part of the barostat protocol. This minimizes the likelihood of inaccurate measurements during the actual procedure and could reduce the procedure time by an additional 10 minutes. Hence, we recommend the correction for RC for visceral perception measurements. The choice of data presentation based either on pressure, corrected for MDP or volume corrected for RC has implications for the individual graphs although the conclusion remained unchanged. Bouin et al. previously described the sensitivity and specificity of pain thresholds in the discrimination between IBS and controls ${ }^{22}$. The sensitivity and specificity to discriminate between healthy and IBS and also between sub-groups of IBS patients applying the barostat protocol as presented here will have to be assessed in follow-up studies. A cut-off score for index volume, as has been done before for pressure to discriminate between hypersensitive and normosensitive subjects needs to be assessed. 


\section{Conclusion}

We have shown that barostat procedures in clinical practice may be shortened without losing the discriminatory value between healthy controls and IBS patients, by measuring compliance during the semi-random part of the protocol, which conventionally was dedicated to assess visceral perception. The total procedure time could be shortened by $20 \mathrm{~min}$ to a total duration of $45 \mathrm{~min}$. The exact duration of the protocol depends on the pressure step at which a patient scores the maximum sensation of pain, urge or discomfort during the perception protocol. An additional advantage of combining these measurements in the same part of the protocol may be that, when corrected for RC, the inter-observer variability may decrease. Validation of this newly proposed procedure is needed in a large group of patients in order to assess its potential and value in a clinical setting. In the near future, consensus should be reached on how to present the data (graphs versus thresholds and volume- versus pressure-based distensions) to enable proper comparison of different studies. 


\section{References}

1. Kilkens TO, Honig A, van Nieuwenhoven MA, Riedel WJ, Brummer RJ. Acute tryptophan depletion affects brain-gut responses in irritable bowel syndrome patients and controls. Gut. 2004;53:1794-800.

2. Kilkens TO, Honig A, Fekkes D, Brummer RJ. The effects of an acute serotonergic challenge on braingut responses in irritable bowel syndrome patients and controls. Aliment Pharmacol Ther. 2005;22:865-74.

3. Ritchie J. Pain from distension of the pelvic colon by inflating a balloon in the irritable colon syndrome. Gut. 1973;14:125-32.

4. Penning C, Steens J, van der Schaar PJ, Kuyvenhoven J, Delemarre JB, Lamers CB, et al. Motor and sensory function of the rectum in different subtypes of constipation. Scand J Gastroenterol. 2001;36:32-8.

5. Steens J, Van Der Schaar PJ, Penning C, Brussee J, Masclee AA. Compliance, tone and sensitivity of the rectum in different subtypes of irritable bowel syndrome. Neurogastroenterol Motil. 2002;14:241-7.

6. Whitehead WE, Holtkotter B, Enck P, Hoelzl R, Holmes KD, Anthony J, et al. Tolerance for rectosigmoid distention in irritable bowel syndrome. Gastroenterology. 1990;98:1187-92.

7. Kanazawa M, Palsson OS, Thiwan SI, Turner MJ, van Tilburg MA, Gangarosa LM, et al. Contributions of pain sensitivity and colonic motility to IBS symptom severity and predominant bowel habits. Am J Gastroenterol. 2008;103:2550-61.

8. Krogh K, Ryhammer AM, Lundby L, Gregersen H, Laurberg TS. Comparison of methods used for measurement of rectal compliance. Dis Colon Rectum. 2001;44:199-206.

9. Fox M, Thumshirn M, Fried M, Schwizer W. Barostat measurement of rectal compliance and capacity. Dis Colon Rectum. 2006;49:360-70.

10. Park JH, Baek YH, Park DI, Kim HJ, Cho YK, Sohn $\mathrm{Cl}$, et al. Analysis of rectal dynamic and static compliances in patients with irritable bowel syndrome. Int J Colorectal Dis. 2008;23:659-64.

11. Whitehead WE, Delvaux M. Standardization of barostat procedures for testing smooth muscle tone and sensory thresholds in the gastrointestinal tract. The Working Team of Glaxo-Wellcome Research, UK. Dig Dis Sci. 1997;42:223-41.

12. Gladman MA, Dvorkin LS, Lunniss PJ, Williams NS, Scott SM. Rectal hyposensitivity: a disorder of the rectal wall or the afferent pathway? An assessment using the barostat. Am J Gastroenterol. 2005;100:106-14.

13. Vanhoutvin SA, Troost FJ, Kilkens TO, Lindsey PJ, Hamer HM, Jonkers DM, et al. The effects of butyrate enemas on visceral perception in healthy volunteers. Neurogastroenterol Motil. 2009;21: 952-e76. .

14. Hammer HF, Phillips SF, Camilleri M, Hanson RB. Rectal tone, distensibility, and perception: reproducibility and response to different distensions. Am J Physiol. 1998;274:G584-90.

15. Ludidi S, Conchillo JM, Keszthelyi D, Koning CJ, Vanhoutvin SA, Lindsey PJ, et al. Does meal ingestion enhance sensitivity of visceroperception assessment in irritable bowel syndrome? Neurogastroenterol Motil. 2012;24:47-53, e3.

16. Akaike H. Information theory and an extension of the maximum likelihood principle. Second international symposium on inference theory (Tsahkadsor, 1971). 1973:267-81.

17. Lindsey PJ, Kaufmann J. Analysis of a longitudinal ordinal response clinical trial using dynamic models. Journal of the Royal Statistical Society Series C-Applied Statistics. 2004;53:523-37.

18. Nozu T, Kudaira M, Kitamori S, Uehara A. Repetitive rectal painful distention induces recta hypersensitivity in patients with irritable bowel syndrome. J Gastroenterol. 2006;41:217-22.

19. Simren M, Agerforz P, Bjornsson ES, Abrahamsson H. Nutrient-dependent enhancement of rectal sensitivity in irritable bowel syndrome (IBS). Neurogastroenterol Motil. 2007;19:20-9.

20. van der Veek PP, Steenvoorden M, Steens J, van der Schaar PJ, Brussee J, Masclee AA. Recto-colonic reflex is impaired in patients with irritable bowel syndrome. Neurogastroenterol Motil. 2007;19: 653-9.

21. Dorn SD, Palsson OS, Thiwan SI, Kanazawa M, Clark WC, van Tilburg MA, et al. Increased colonic pain sensitivity in irritable bowel syndrome is the result of an increased tendency to report pain rather than increased neurosensory sensitivity. Gut. 2007;56:1202-9. 
22. Bouin $M$, Plourde $V$, Boivin $M$, Riberdy $M$, Lupien $F$, Laganiere $M$, et al. Rectal distention testing in patients with irritable bowel syndrome: sensitivity, specificity, and predictive values of pain sensory thresholds. Gastroenterology. 2002;122:1771-7. 


\section{S6.1 Statistical analysis}

All data analyses take the experimental design into account as to provide the most appropriate analysis.

\section{Minimal distension pressure (MDP) and first sensation (FS) data analysis}

MDP and FS were each analysed using a Gaussian linear regression ( $\mathrm{N}\left(\mu, \sigma^{2}\right)$ where $\mu$ is the mean and $\sigma^{2}$ is the variance). For both analyses, the body mass index (BMI), FS, and compliance (COMP) were included in the model.

The inference criterion used for comparing the models is their ability to predict the observed data, i.e. models are compared directly through their minimized minus loglikelihood. When the numbers of parameters in models differed, they were penalized by adding the number of estimated parameters, a form of the Akaike information criterion $(\mathrm{AIC})^{16}$.

For each variable of interest, a model containing the relevant covariates mentioned above ( $\mathrm{E}(y)=\beta_{0}+\beta_{1} \times \mathrm{BMI}+\beta_{2} \times \mathrm{FS}+\beta_{3} \times \mathrm{COMP}$ ) was fitted in order to obtain a reference AIC. Then a model containing the group was fitted ( $\mathrm{E}(y)=\beta_{0}+\beta_{1} \times \mathrm{BMI}+\beta_{2} \times \mathrm{FS}+\beta_{3} \times \mathrm{COMP}+\beta_{4} \times \mathrm{Grp}$ ).

The variable of interest was found to be differentially expressed if the AIC of the model containing a group effect was smaller than the reference AIC (the model not containing the group effect).

MDP, BMI, and rectal capacity (RC) were also analysed by bivariate Gaussian linear regression ( $\mathrm{BVN}(\mu, \Sigma)$ where $\mu$ is the mean, $\sum$ is the two-by-two covariance matrix $\left(\begin{array}{cc}\sigma^{2}+\delta & \delta \\ \delta & \sigma^{2}+\delta\end{array}\right), \sigma^{2}$ is the variance and $\delta$ as both the extra component

of variance across subjects and the common covariance among responses on the same subject) including the appropriate covariance structure in order to capture the dependence between them. The compliance and FS were included as explanatory variables. As for the previously described analysis, the AIC was used to assess a group effect.

\section{Rectal capacity data analysis}

The RC volume was analyzed using a Gaussian non-linear regression ( $N\left(\mu, \sigma^{2}\right)$ where $\mu$ is the mean and $\sigma^{2}$ is the variance) including the pressure and compliance as explanatory variables. Again, the AIC was used to assess whether there was a group effect. 


\section{Visceral perception data analysis}

Pain and discomfort data were analyzed using a multivariate Gaussian non-linear regression ( $\mathrm{MVN}(\mu, \Sigma)$ where $\mu$ is the mean, $\sum$ is the covariance matrix

$$
\left(\begin{array}{cccc}
\sigma^{2}+\delta & \delta+\rho^{t_{2}-t_{1}} & \cdots & \delta+\rho^{t_{n}-t_{1}} \\
\delta+\rho^{t_{2}-t_{1}} & \ddots & \ddots & \vdots \\
\vdots & \ddots & \ddots & \delta+\rho^{t_{n}-t_{n-1}} \\
\delta+\rho^{t_{n}-t_{1}} & \cdots & \delta+\rho^{t_{n}-t_{n-1}} & \sigma^{2}+\delta
\end{array}\right), \sigma^{2} \text { is the variance, } \rho^{t_{n}-t_{n-1}} \text { is }
$$

the first order autocorrelation taking the time lag with the previous observation into account, and $\delta$ as both the extra component of variance across subjects and the common covariance among responses on the same subject) including, if necessary, a random effect and a first order autocorrelation.

Urge was scored on an ordinal 6-point scale and was analyzed using a mixture of a logistic distribution (parameterized as a proportional-odds) and a gamma distribution (to introduce frailty and autocorrelation dependencies) ${ }^{17}$. The first model was obtained by imposing the mean regression to follow a logistic ('S-shape') curve through the pressure variable $\left(\mathrm{E}(y)=\beta_{0} /\left(1+e^{\left(\beta_{1}+\beta_{2} \times \text { Pressure }\right)}\right)\right)$. Then models including MDP and FS as explanatory variables were build $\left(\mathrm{E}(y)=\beta_{0} /\left(1+e^{\left(\beta_{1}+\beta_{2} \times \text { Pressure }+\beta_{3} \times \mathrm{MDP}+\beta_{4} \times \mathrm{FS}\right)}\right)\right)$ in order to obtain a reference AIC.

114 Finally, a model containing the group effect was fitted and the AIC was used to assess this group effect was significant. 

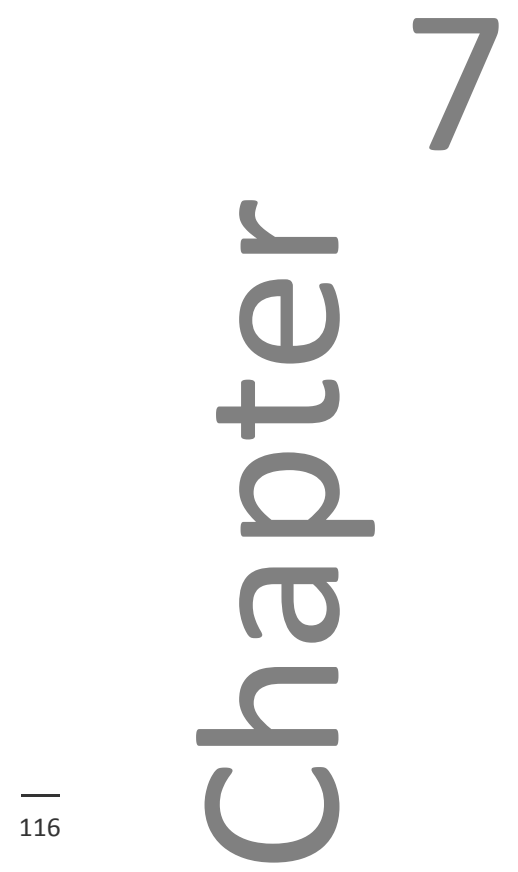
Summary and general discussion 


\section{Summary and general discussion}

Short chain fatty acids (SCFAs) are produced via fermentation of non digestible carbohydrates in the colon. Of the SCFAs, butyrate is generally considered to exert the most potent favourable effects on colonic function and gut health.

The typical Western diet does not contain adequate amounts of fibers to contribute to optimal gut health ${ }^{1}$. Insufficient fiber intake results in suboptimal saccharolytic fermentation in the colon, while the rate of proteolytic fermentation consequently is high. The potentially toxic products of proteolytic fermentation are thought to play a role in several colon disorders, such as colorectal cancer ${ }^{2}$. In contrast, increased intracolonic butyrate production is generally assumed to be beneficial to gut health and may even have therapeutic potential in several gastrointestinal disorders. Up to now, there is limited scientific evidence to substantiate potential health effects of SCFA in vivo in humans. Research on the effects of SCFA in vivo has been hampered by the relative inaccessibility of the colon to administer substrates, and to collect biological samples. A reliable and preferably minimally invasive delivery technique is required to deliver substrates to the colon without compromising colon physiology. In this thesis a selection of potential delivery techniques (i.e. enteric coated capsules, oro-cecal catheters and enemas) have been tested in explorative studies described in chapter 2. The enteric coated capsules proved to be reliable for delivery in the terminal ileum/proximal colon in $85 \%$ of the cases. However, application of this technique in a research setting, irrespective of the observed moderate variation in delivery location, is limited by the rather small amount of test substance that can be transported to the target location. A large number of capsules would be required to administer sufficient amounts of substrate, thereby increasing the risk of unsuccessful delivery to the target location.

The oro-ileal catheter does not pertain the disadvantage of limited substrate delivery and the delivery location can be determined quite accurately. Additionally, a multilumen catheter provides the unique feature of sampling intraluminal fluid contents at various intestinal locations. Two important drawbacks of this technique are first; the time consuming procedure of catheter positioning that occurs through peristalsis and the fact that after successful positioning of the catheter tip into the terminal ileum, the tip of the catheter may progress through the ileocecal valve into the colon. Second; repeated fluoroscopic imaging is necessary to monitor the progress of the tip of the catheter.

The use of rectally administered enemas allowed delivery of sufficient amounts of substrates to the sigmoid region and also to part of the descending colon and was well tolerated by the subjects. Although bowel movements influenced optimal spread of enema contents, the $60 \mathrm{ml}$ enemas reached a region as far as the descending colon, in an unprepared colon. Another advantage of this method is that volunteers can self- 
administer the enemas at home, lowering the need for hospital visits, not requiring the use of dedicated equipment or assistance of trained personnel.

Due to the reliability of the enemas and the fact that enema administration is very patient friendly with low burden, this method has been applied in several studies of the present thesis. In chapter $\mathbf{3}$ we reported the results of a study on the effects of rectally administered butyrate on gene expression in colonic mucosa. In total 501 genes were differentially expressed after butyrate treatment. Butyrate significantly modulated several pathways such as transport of fatty acids, beta-oxidation and the electron transport chain, which are all pathways involved in the metabolic processing of butyrate. This suggests an increased uptake and metabolism of butyrate. In an inflamed colon, reactive oxygen species (ROS) are produced. These highly reactive radicals induce oxidative damage to organic molecules when ROS production exceeds the antioxidative capacity at the site of origin. This has previously been reported in patients with ulcerative colitis $(U C)^{3}$. Butyrate was found to upregulate the gene "nuclear factor kappa beta inhibitor alpha (NFkBIA)", which inhibits the activation of NF- $k B$ and the TNF-alpha cascade and potentially diminishes inflammation and inflammation-induced oxidative stress. In the oxidative stress pathway, a number of genes involved in glutathione metabolism were differentially expressed, indicating an increased glutathione turnover and increased antioxidant capacity. Related to this, butyrate significantly modulated the pathway "proteosome degradation" in which eight genes were differentially expressed. This pathway provides a mechanism for the degradation of (oxidatively) damaged proteins and the genes in this pathway are associated with oxidative stress, apoptosis and ageing. These findings were in line with previously reported results from our group, describing the effect of butyrate on oxidative stress parameters ${ }^{4}$. The results of the present in vivo intervention study clearly support previously described findings, that the beneficial effects of butyrate can mainly be attributed to modulation of inflammation, oxidative stress and apoptosis.

Butyrate is thought to improve and strengthen intestinal barrier function, by modulation of tight junction expression ${ }^{5-8}$. An increased intestinal permeability has implications for pathogen infiltration, which may result in activation of the local intestinal immune system and, in the worst case, in mucosal inflammation. Intestinal permeability of inert permeation markers was shown to be increased in several conditions, such as UC and irritable bowel syndrome (IBS) ${ }^{9}$. These findings support the association between intestinal barrier dysfunction and mucosal immune activation and mucosal inflammation. Increased intestinal permeability may allow noxious substances to pass the epithelial barrier. These substances may trigger enterig afferent neurons projecting to the CNS or induce local inflammatory and immune responses. The established anti-inflammatory capacity of butyrate ${ }^{10}$, and the putative relationship between permeability, inflammation and functional bowel diseases, led 
to the studies described in chapters 4 and 5, respectively. In these studies, the effects of intracolonic butyrate on visceral perception were studied in healthy volunteers and in IBS patients, respectively. In a placebo controlled study in healthy volunteers (chapter 4), administration of both 50 and $100 \mathrm{mM}$ butyrate once daily for a week significantly decreased sensations of pain, urge and discomfort. The observed effects were dose dependent. Furthermore, butyrate increased rectal compliance, indicating that relaxation of the rectal wall increased in response to the increasing balloon pressures after butyrate treatment.

In a follow-up study in IBS patients (chapter 5), only the high concentration of 100 $\mathrm{mM}$ butyrate was tested. In those patients, butyrate significantly decreased sensations of pain and urge, but did not change rectal compliance or feelings of discomfort. The effects of butyrate on pain and urge scores suggest a potential role for butyrate or a food intervention resulting in increased butyrate production in the treatment of IBS related symptoms.

In that study the potential preventive effect of butyrate on deoxycholate-induced increase in intestinal permeability was examined in an ex vivo model using human intestinal tissue samples. The bile acid deoxycholate negatively affected intestinal barrier function. Although butyrate dose dependently improved mucosal barrier function, the effect was not statistically significant due to small sample size and large inter-individual variation. Although the ex vivo experiment was not performed in tissue from the IBS patients to prevent interaction between the biopsies and sensitivity scores, these findings support previous observations that butyrate may improve gut permeability ${ }^{6-11,12}$. Changes in gut permeability are considered to be an early event in the development of several GI and systemic disorders and an association between permeability, visceroperception and IBS has previously been hypothesized $^{13-15}$. Consequently, the results of the studies described in chapters $\mathbf{4}$ and 5 provide a new lead in the treatment of barrier dysfunction and visceral hypersensitivity in IBS patients. In the current study, due to invasiveness we did not assess permeability in the same patient group.

The barostat procedure applied in these studies is a widely used technique to assess visceral perception. However, this technique still lacks consensus on the exact duration and intensity of pressure steps, outcome parameters and data analysis for correction of inter-individual variation. This hampers comparison of outcomes of the reported studies in the literature. In chapter 6 we propose a new, short and les complex protocol that still permits us to distinguish between healthy volunteers and IBS patients but is less prone to inter-observer variation in assessing minimal distension pressure during the procedure. 


\section{Future perspectives}

Because of the difficulty to access the human colon for non-diagnostic or therapeutic interventions, the need to find non-invasive routes for delivery of substrates such as butyrate to the human colon remains challenging. When targeting the proximal colon, the technology of naso-intestinal catheters should be optimized for instance by using non-invasive positioning tools such as electromagnetic imaging to assess catheter localization in vivo, as an alternative for fluoroscopy. Another goal is to optimize catheter design and construction by adequate catheter length and stiffness in order to minimize subject discomfort and maximize positioning success rate. Although rectal enemas have been proven to provide a reliable and suitable tool for selfadministration of substrates to the rectum, the ultimate goal should be to study the effects of dietary interventions with dietary substrates (such as dietary fibers) that result in increased concentrations of SCFAs, especially butyrate, in the colon. Eventually, the currently available scientific data, including those presented in this thesis, point to a clear need to develop dietary strategies that lead to increased colonic butyrate concentrations.

Previous studies have pointed to differences in butyrate production as result of microbial fermentation of various dietary fibers. In general, soluble fibers are fermented to a much greater extent than non-soluble fibers. Most prebiotic fibers such as fructo-oligosaccharides (FOS), inulin, galacto-oligosaccharides (GOS) and xylooligosaccharides (XOS), all with different degrees of polymerisation, have been shown to increase fecal counts of bifidobacteria ${ }^{16-19}$. Although bifidobacteria do not produce butyrate directly, an increased number of bifidobacteria could result in an increased butyrate production via crossfeeding, indicating that some butyrate producing species feed on metabolic endproducts of others. For example, Eubacterium hallii utilizes lactate, which is formed by the metabolism of a variety of microbes, and produces butyrate $^{20}$. It would be of interest to develop relatively slow fermentable fibers with high degree of polymerization subsequently leading to higher butyrate concentrations in the distal part of the gut. Inulin has been reported to increase butyrate production by colonic bacteria ${ }^{21}$. Despite the beneficial effects of prebiotics, such as butyrate production and/or increasing the number of bifidobacteria, not all dietary fibers are well tolerated when supplemented. Steward et al. reported the effects of dietary supplementation with $12 \mathrm{~g}$ of different dietary fibers (pullulan, resistant starch, dextrin or soluble corn fiber). All four fibers were well tolerated with only mild or moderate GI symptoms ${ }^{22}$. In IBS patients however, the beneficial effect of dietary supplementation is questionable since inulin but also fermentable oligo-, di- and mono-saccharides and polyols (FODMAPs) were reported to induce symptoms like flatulence ${ }^{23-25}$.

Recently Clarke et al. have taken a different approach and showed that butyrylated starch is effective in increasing the concentration of butyrate in the colon in both rats 
and humans. Butyrylated starch consists of starch with a high number of butyric acid molecules esterified to the branches. When degraded, the butyrate is released and contributes, in addition to the butyrate that is produced during the fermentation of the starch molecules itself, to increased intracolonic butyrate concentrations. This was found to provide a dietary tool to increase intracolonic butyrate concentrations without inducing gastrointestinal side effects ${ }^{26}$. An alternative approach to establish butyrate induced health effects is to modulate the composition of microbiota in favour of growth of butyrate producing microbes, by supplementation of specific fibers. Known butyrate producing bacteria include different Clostridium species, Faecalibacterium prausnitzii and Eubacterium rectale that either produce butyrate directly via the fermentation of carbohydrate sources or by means of crossfeeding ${ }^{27}$.

In anticipation of dietary intervention studies which aim to improve gut health by increasing butyrate levels in the proximal as well as the distal colon, more information is needed on the dose-response effects of butyrate to establish the optimal concentration and duration for butyrate administration. The beneficial effect of butyrate shown in IBS patients with respect to visceral sensitivity are promising and may hold therapeutic potential with respect to improving gastrointestinal symptoms. More research in a larger IBS population is needed to identify the minimally effective and optimal dose of butyrate to significantly reduce clinical symptoms. Preferably, in order to lower the heterogenity of the population, follow-up studies should focus on assessing the effects of butyrate on visceral sensitivity and gastrointestinal symptoms in patients suffering from different IBS sub-types based for example on predominant bowel habits or hypersensitivity versus normosensitivity). Meanwhile, intestinal metabolic processing of fibers and of butyrate should be included.

The effects of butyrate enemas in UC patients have been studied previously in intervention studies with a duration of butyrate administration up to 8 weeks [10]. Previous studies in patients suffering from acute post radiation proctitis suggest beneficial effects during treatment with butyrate enemas but those effects disappeared when butyrate administration was stopped with no effect of butyrate treatment on the incidence of chronic radiation proctitis ${ }^{28}$. This observation indicates that also in other populations such as IBS patients, a continuous butyrate supplementation may be necessary for a sustained effect. Partly due to the longer term duration of supplementation that is required, future applications should come from dietary additives/interventions instead of applying enemas or by using other medical interventions tools. These studies should not focus on mechanistic factors only but also on clinical outcome parameters such as disease activity, functional abdominal complaints, quality of life and health care consumption. If possible and feasible, mechanistic evaluations should be included. With a prevalence of up to $15 \%$ in the Dutch population and significant associated health care costs and a diminished quality of life, at population and health care economic level, IBS is major target for 
health care cost reduction programs and also a target for improvement in symptom relief.

In chapter 3, we confirmed the capacity of butyrate to control pivotal cellular processes, such as energy metabolism, fatty acid metabolism, apoptosis, proteasome degradation and oxidative stress. Taken together, these data clearly support the hypothesis that increasing colonic butyrate levels may provide a relevant nutritional strategy in the treatment of conditions that are associated with mucosal inflammation, increased oxidative stress and disturbances in cellular metabolism and immune activation. It is therefore of outmost importance to study the effects of increasing colonic butyrate levels, preferably to be reached via dietary intervention. This will potentially lead to therapeutic strategies for conditions or disorders associated with mild impairments in immune activation and oxidative capacity such as IBS, or as maintenance therapy in IBD patients in remission.

Future studies should focus on the development of food additives or food supplements that are readily available at population level and are capable of increasing colonic butyrate concentrations. The use of an optimized pre- or synbiotic agent, possibly combined the butyrate esterification technology, has the advantage of not only large-scale production potential at low cost, but also release of butyrate in the colon without the typical unpleasant odor of butyrate of the dietary supplement or food additive. In parallel, alternative ways should be explored to optimize butyrate concentrations in the colon via dietary intervention for a variety of patients suffering from a gastrointestinal and even systemic disorders. 


\section{References}

1. Slavin J. Fiber and prebiotics: mechanisms and health benefits. Nutrients 2013;5:1417-35.

2. Le Leu RK, Young GP. Fermentation of starch and protein in the colon: implications for genomic instability. Cancer biology \& therapy 2007;6:259-60.

3. Kruidenier L, Kuiper I, Lamers CB, et al. Intestinal oxidative damage in inflammatory bowel disease: semi-quantification, localization, and association with mucosal antioxidants. The Journal of pathology 2003;201:28-36.

4. Hamer HM, Jonkers DM, Bast A, et al. Butyrate modulates oxidative stress in the colonic mucosa of healthy humans. Clinical nutrition (Edinburgh, Scotland) 2009;28:88-93.

5. Bordin M, D'Atri F, Guillemot L, et al. Histone deacetylase inhibitors up-regulate the expression of tight junction proteins. Mol Cancer Res 2004;2:692-701.

6. Ohata A, Usami M, Miyoshi M. Short-chain fatty acids alter tight junction permeability in intestinal monolayer cells via lipoxygenase activation. Nutrition 2005;21:838-47.

7. Peng L, Li ZR, Green RS, et al. Butyrate enhances the intestinal barrier by facilitating tight junction assembly via activation of AMP-activated protein kinase in caco-2 cell monolayers. J Nutr 2009; 139:1619-25.

8. Wang $\mathrm{HB}$, Wang PY, Wang $X$, et al. Butyrate enhances intestinal epithelial barrier function via upregulation of tight junction protein Claudin-1 transcription. Dig Dis Sci 2012;57:3126-35.

9. Vivinus-Nebot $M$, Frin-Mathy $G$, Bzioueche $\mathrm{H}$, et al. Functional bowel symptoms in quiescent inflammatory bowel diseases: role of epithelial barrier disruption and low-grade inflammation. Gut 2014;63:744-52

10. Hamer HM, Jonkers D, Venema K, et al. Review article: the role of butyrate on colonic function. Aliment Pharmacol Ther 2008;27:104-19.

11. Suzuki T, Yoshida S, Hara H. Physiological concentrations of short-chain fatty acids immediately suppress colonic epithelial permeability. Br J Nutr 2008;100:297-305.

12. Ploger S, Stumpff F, Penner GB, et al. Microbial butyrate and its role for barrier function in the gastrointestinal tract. Ann N Y Acad Sci 2012;1258:52-9.

13. Annahazi A, Ferrier L, Bezirard V, et al. Luminal cysteine-proteases degrade colonic tight junction structure and are responsible for abdominal pain in constipation-predominant IBS. Am J Gastroenterol 2013;108:1322-31.

14. Piche $T$, Barbara $G$, Aubert $P$, et al. Impaired intestinal barrier integrity in the colon of patients with irritable bowel syndrome: involvement of soluble mediators. Gut 2009;58:196-201.

15. Turcotte JF, Kao D, Mah SJ, et al. Breaks in the wall: increased gaps in the intestinal epithelium of irritable bowel syndrome patients identified by confocal laser endomicroscopy (with videos). Gastrointest Endosc 2013;77:624-30.

16. Lecerf JM, Depeint F, Clerc E, et al. Xylo-oligosaccharide (XOS) in combination with inulin modulates both the intestinal environment and immune status in healthy subjects, while XOS alone only shows prebiotic properties. Br J Nutr 2012;108:1847-58.

17. Macfarlane GT, Steed H, Macfarlane S. Bacterial metabolism and health-related effects of galactooligosaccharides and other prebiotics. Journal of applied microbiology 2008;104:305-44.

18. Ramirez-Farias C, Slezak K, Fuller Z, et al. Effect of inulin on the human gut microbiota: stimulation of Bifidobacterium adolescentis and Faecalibacterium prausnitzii. Br J Nutr 2009;101:541-50.

19. Roberfroid MB, Van Loo JA, Gibson GR. The bifidogenic nature of chicory inulin and its hydrolysis products. J Nutr 1998;128:11-9.

20. Duncan $\mathrm{SH}$, Louis $\mathrm{P}$, Flint HJ. Lactate-utilizing bacteria, isolated from human feces, that produce butyrate as a major fermentation product. Appl Environ Microbiol 2004;70:5810-7.

21. Kleessen B, Hartmann L, Blaut M. Oligofructose and long-chain inulin: influence on the gut microbial ecology of rats associated with a human faecal flora. Br J Nutr 2001;86:291-300.

22. Stewart ML, Nikhanj SD, Timm DA, et al. Evaluation of the effect of four fibers on laxation, gastrointestinal tolerance and serum markers in healthy humans. Annals of nutrition \& metabolism 2010;56:91-8. 
23. Ong DK, Mitchell SB, Barrett JS, et al. Manipulation of dietary short chain carbohydrates alters the pattern of gas production and genesis of symptoms in irritable bowel syndrome. J Gastroenterol Hepatol 2010;25:1366-73.

24. Ramnani $P$, Gaudier $E$, Bingham $M$, et al. Prebiotic effect of fruit and vegetable shots containing Jerusalem artichoke inulin: a human intervention study. Br J Nutr 2010;104:233-40.

25. Staudacher HM, Whelan K, Irving PM, et al. Comparison of symptom response following advice for a diet low in fermentable carbohydrates (FODMAPs) versus standard dietary advice in patients with irritable bowel syndrome. J Hum Nutr Diet 2011;24:487-95.

26. Clarke JM, Topping DL, Christophersen CT, et al. Butyrate esterified to starch is released in the human gastrointestinal tract. Am J Clin Nutr 2011;94:1276-83.

27. Flint HJ, Duncan SH, Scott KP, et al. Interactions and competition within the microbial community of the human colon: links between diet and health. Environmental microbiology 2007;9:1101-11.

28. Hille A, Herrmann MK, Kertesz $\mathrm{T}$, et al. Sodium butyrate enemas in the treatment of acute radiationinduced proctitis in patients with prostate cancer and the impact on late proctitis. A prospective evaluation. Strahlenther Onkol 2008;184:686-92. 
Samenvatting 


\section{Samenvatting}

Korte keten vetzuren worden gevormd tijdens de fermentatie van niet-verteerbare koolhydraten in het colon. Met name het korte keten vetzuur butyraat wordt in verband gebracht met gunstige effecten op darmgezondheid.

Ons Westers dieet bevat onvoldoende voedingsvezels voor het bewerkstelligen van een optimale productie van korte keten vetzuren, teneinde positieve effecten op darmgezondheid te verkrijgen. De lage vezelinname leidt enerzijds tot een verminderde fermentatie van koolhydraten (saccharolytische fermentatie) maar zorgt er ook voor dat er eerder en meer fermentatie van eiwitten (proteolytische fermentatie) plaatsvindt als alternatief voor de gezonder geachte saccharolytische fermentatie. De producten die gevormd worden bij de fermentatie van eiwitten bestaan deels uit toxische stoffen waarvan gedacht wordt dat deze een rol spelen bij het ontstaan van verschillende darmaandoeningen, waaronder colonkanker.

Aangenomen wordt dat een verhoogde productie van butyraat in het colon gunstig is voor darmgezondheid en wellicht zelfs ingezet kan worden als potentiele therapie bij verschillende darmaandoeningen. Tot nu toe is er beperkt bewijs voor substantiële effecten van korte keten vetzuren in vivo in mensen. In vivo onderzoek naar de effecten van korte keten vetzuren in het colon is tot op heden gehinderd door de relatief moeilijke bereikbaarheid van het colon om substraten toe te dienen en weefsel- en vloeistofmonsters af te nemen. Een betrouwbare en minimaal invasieve toedieningsvorm is nodig om testsubstraten in het colon te brengen zonder de fysiologische omstandigheden te verstoren.

In hoofdstuk 2 van dit proefschrift is een selectie van potentieel haalbare toedieningstechnieken getest in een serie kleine interventiestudies. Het betreft het gebruik van gecoate capsules, een oro-caecale katheter en klysma's.

Het onderzoek met de capsules, met pH gevoelige coating, heeft aangetoond dat de capsules in $85 \%$ van de gevallen desintegreren in het terminale ileum of proximale colon. Het gebruik van deze capsules wordt echter beperkt door de beperkte hoeveelheid substraat die zij kunnen bevatten. Voor veel interventiestudies zou een groot aantal capsules nodig zijn om voldoende substraat gericht naar het doelgebied (het proximale colon) te brengen. Door de vastgestelde spreiding in locatie van desintegratie van de capsules betekent dit dat een aanzienlijk deel van het toe te dienen substraat buiten het doelgebied beschikbaar komt, hetgeen onwenselijk is.

De oro-caecale katheter heeft dit nadeel niet en kan een bijna ongelimiteerde hoeveelheid substraat met grote precisie in het colon brengen. Daarbij heeft het gebruik van een katheter het grote voordeel dat niet alleen vloeistof toegediend kan worden maar dat ook het afnemen van darmvloeistof op verschillende locaties mogelijk is. Een groot nadeel van deze techniek is de tijd die nodig is om de katheter via de normale peristaltiek te positioneren en het feit dat het moeilijk is om wanneer eenmaal bereikt, de katheter op de gewenste positie te behouden. De katheter wordt vrij makkelijk verder naar het colon gevoerd gedurende het experiment. Bovendien 
ondervinden proefpersonen hinder van de aanwezigheid van de sonde in neus- en keelholte.

Het gebruik van klysma's geeft de mogelijkheid om voldoende hoeveelheden substraat in het rectum, sigmoïd en colon te brengen. Bovendien wordt het gebruik van klysma's goed verdragen. Hoewel de peristaltiek de maximale verspreiding van de klysmavloeistof beïnvloedde werd met deze 60ml klysma's in een onvoorbereide darm het dalende deel van het colon bereikt. Een ander voordeel van het gebruik van klysma's is dat het gebruik van deze toedieningsmethode toelaat dat vrijwilligers en patiënten de klysma's zelf thuis over langere tijdsperioden toe kunnen dienen. Hierdoor is het tijdens de uitvoering van langdurige experimenten met het gebruik van klysma's niet noodzakelijk dat proefpersonen gedurende langere tijd binnen de onderzoeksfaciliteit verblijven.

Vanwege de hoge betrouwbaarheid en het grote gebruiksgemak van deze toedieningsmethode is voor de latere studies beschreven in dit proefschrift gebruik gemaakt van klysma's voor de toediening van butyraat.

In hoofdstuk 3 zijn de effecten van butyraat, toegediend via klysma's, op de genexpressie in colon mucosa beschreven. In totaal werd van 501 genen een veranderde transcriptie aangetoond na de behandeling met butyraat. Butyraat beïnvloedt de aansturing van verschillende biologische processen, waaronder met name het transport van vetzuren, beta-oxidatie en de elektronen transportketen, allen betrokken bij het metaboliseren van het toegediende butyraat. Dit wijst op een versnelde opname en metabolisme van butyraat.

In een ontstoken darm worden grote hoeveelheden vrije radicalen gevormd. Deze stoffen zijn zeer reactief en veroorzaken oxidatieve schade aan organische moleculen wanneer de hoeveelheid groter wordt dan de antioxidant capaciteit het weefsel op die plaats. In patiënten met colitis ulcerosa is dit eerder aangetoond ${ }^{1,2}$. Butyraat zorgde voor een toename in de transcriptie van het gen "nuclear factor kappa beta inhibitor alpha (NFkBIA)" dat de activatie van NF-kB en het TNF-alpha proces remt en daarmee mogelijk inflammatie en inflammatie-geïnduceerde oxidatieve stress verlaagt. Bovendien werd een aantal genen die een rol spelen bij de omzetting van het antioxidant glutathion gereguleerd. Dit duidt erop dat via de verhoogde glutathion-omzetting de antioxidant capaciteit van het weefsel wordt verhoogd.

Onder invloed van butyraat werd tevens het proces "proteosome degradation" tot expressie gebracht. Dit proces omhelst een mechanisme voor de afbraak van (eventueel door oxidatieve stress) beschadigde eiwitten. De genen in dit proces zijn geassocieerd met oxidatieve stress, apoptose en veroudering. De resultaten van deze in vivo interventiestudie ondersteunen eerder beschreven gunstige effecten van butyraat, met name op het gebied van inflammatie, oxidatieve stress en apoptose.

De reeds eerder beschreven effecten van butyraat op het versterken van de integriteit van de darmwand worden onder andere toegeschreven aan het feit dat butyraat een 
direct effect zou hebben op het reguleren van tight junction-geassocieerde eiwitten ${ }^{3,4}$. Tight junctions zijn verbindende structuren in de darmwand, die belangrijk zijn voor het in stand houden van een goede barrièrefunctie van de darm tegen het binnendringen van ziekteverwekkende of irriterende stoffen. Een toegenomen doorlaatbaarheid van de darm heeft nadelige gevolgen voor de infiltratie van pathogene bacterieën die het immuunsysteem kunnen activeren en voor lokale ontsteking in de darm kunnen zorgen. Uit eerdere studies weten we dat in ziektes die gepaard gaan met ontsteking zoals colitis ulcerosa en prikkelbare darm syndroom (IBS), de doorlaatbaarheid van de darm voor inerte markers is verhoogd ${ }^{5,6}$. Deze bevindingen ondersteunen het bestaan van een verband tussen een verstoorde barrièrefunctie en mucosale ontsteking. De eerder aangetoonde anti-inflammatoire capaciteit van butyraat ${ }^{7}$, en de veronderstelde relatie tussen doorlaatbaarheid van de darmwand, inflammatie en IBS resulteerden in de studies zoals beschreven in hoofdstukken 4 en 5 . In deze studies werden de effecten van een dagelijkse toediening van butyraatklysma's gedurende een week op viscerale perceptie in gezonde vrijwilligers en in IBS patiënten onderzocht.

In een placebo gecontroleerde gerandomiseerde crossover studie in gezonde vrijwilligers induceerden zowel $50 \mathrm{mM}$ als $100 \mathrm{mM}$ butyraat een significante en concentratie afhankelijke vermindering van de scores voor pijn, aandrang en ongemak tijdens de barostat onderzoeken. Daarnaast verhoogde butyraat de rectale compliantie, hetgeen duidt op een verhoogde relaxatie van de darmwand in respons op de toenemende druk van de barostatballon in het rectum.

In de daaropvolgende studie in IBS patiënten werd alleen de hoogste concentratie van $100 \mathrm{mM}$ butyraat getest. Butyraat zorgde in deze patiëntengroep voor een significante verlaging van pijn- en aandrang scores. De interventie had geen effect op de scores voor ongemak en op de rectale compliantie.

De effecten van butyraat op pijn en aandrang scores duiden op een mogelijke rol van butyraat of een voedingsinterventie leidend tot een verhoogde butyraat productie in de behandeling van IBS gerelateerde symptomen. In dit hoofdstuk is ook een mechanistisch onderzoek beschreven waarin de effecten van butyraat op de verhoogde intestinale permeabiliteit, veroorzaakt door het galzuur deoxycholaat, werden onderzocht. In het ex vivo model dat hiervoor gebruikt werd zijn weefselmonsters uit het colon blootgesteld aan deoxycholaat, in aan- en afwezigheid van verschillende concentraties butyraat. Deochycholaat zorgde voor een toename van de mucosale doorlaatbaarheid, gemeten met behulp van de flux van de inerte marker sucralose. Hoewel pre-incubatie met butyraat deze toegenomen permeabiliteit dosis afhankelijk kon voorkomen was dit effect niet significant vanwege de te kleine aantallen en een te grote spreiding in de resultaten. De bevindingen ondersteunen wel eerdere observaties dat butyraat de barrièrefunctie van de darm kan verbeteren ${ }^{8}$ en bieden daardoor een nieuw aanknopingspunt voor de behandeling van hypersensitiviteit bij IBS patiënten. 
De barostat procedure voor het meten van de viscerale gevoeligheid zoals in deze studies is gebruikt is een algemeen geaccepteerde en wereldwijd gebruikte techniek. Toch is er nog steeds gebrek aan consensus over de opbouw van het protocol en de presentatie van de uitkomstmaten waardoor de vergelijking tussen verschillende onderzoeken lastig is. In hoofdstuk 6 wordt een nieuw voorstel voor de opzet van barostat onderzoeken gepresenteerd, waarin het bestaande protocol wordt verkort en vereenvoudigd. Hierdoor is het barostat-protocol minder gevoelig voor fouten en variaties tijdens de uitvoering van het onderzoek.

Toekomstig onderzoek zou zich moeten focussen op de ontwikkeling van voedingssupplementen die al beschikbaar zijn voor het grote publiek en in staat zijn om de concentratie butyraat in het colon te verhogen. Het gebruik van een geoptimaliseerd pre- of probioticum, mogelijk gecombineerd met het gebruik van de techniek voor het veresteren van butyraatmoleculen, heeft niet alleen het voordeel dat het relatief goedkoop op grote schaal geproduceerd kan worden, maar ook dat het een extra verhoging van de butyraatconcentratie in het colon kan bewerkstelligen zonder bij inname met de penetrante geur van vrij butyraat te worden geconfronteerd.

Daarnaast zou de effectiviteit van alternatieve behandelmethoden met behulp van genoemde dieetinterventies die leiden tot een toename in de butyraat concentratie in het colon voor verschillende patientengroepen met een gastrointestinale of systemische ziekte moeten worden onderzocht. 


\section{Referenties}

1. Kruidenier L, Kuiper I, Lamers CB, Verspaget HW. Intestinal oxidative damage in inflammatory bowel disease: semi-quantification, localization, and association with mucosal antioxidants. J Pathol 2003;201:28-36.

2. Pravda J. Radical induction theory of ulcerative colitis. World J Gastroenterol 2005;11:2371-2384.

3. Wang HB, Wang PY, Wang X, Wan YL, Liu YC. Butyrate enhances intestinal epithelial barrier function via up-regulation of tight junction protein Claudin-1 transcription. Dig Dis Sciences 2012;57:3126-35.

4. Bordin M, D'Atri F, Guillemot L, Citi S. Histone deacetylase inhibitors up-regulate the expression of tight junction proteins. Mol Cancer Res 2004;2:692-701.

5. Camilleri M, Gorman H. Intestinal permeability and irritable bowel syndrome. Neurogastroenterol Motil 2007; 19:545-52.

6. Lee H, Park JH, Park DI, Kim HJ, Cho YK, Sohn Cl, Jeon WK, Kim BI, Chae SW. Mucosal mast cell count is associated with intestinal permeability in patients with diarrhea predominant irritable bowel syndrome. J Neurogastroenterol Motil 2013;19:244-50.

7. Hamer HM, Jonkers D, Venema K, Vanhoutvin S, Troost FJ, Brummer RJ. Review article: the role of butyrate on colonic function. Aliment Pharmacol Ther 2008;27:104-19.

8. Suzuki T, Yoshida S, Hara H. Physiological concentrations of short-chain fatty acids immediately suppress colonic epithelial permeability. Br J Nutr 2008;100:297-305. 
Valorisation 


\section{Valorisation}

The gut is considered to be the gateway to health but also is a gateway to several gastrointestinal and systemic disorders. The incidence of gastrointestinal and liver diseases is rapidly expanding. This is especially true in the aging population.

The Dutch society and its economy are highly knowledge based, meaning that the translation of scientific results into commercially attractive products with clear benefits for patients or consumers in daily life is stimulated. The study outcomes presented in this thesis pave the way for development of novel preferably low-cost nutrition based interventions in the management of highly prevalent gastrointestinal disorders with invalidating symptoms.

\section{Relevance}

Clinical or mechanistic studies in humans are demanding, time comsuming and very expensive when compared to experiments in animal models or in vitro laboratory models or cell culture systems. It should be acknowledged that results from research in animals or in vitro tests cannot easily be translated to the human situation. The studies that have been presented in this thesis describe effects of interventions in vivo in humans, both in healthy volunteers and in patients. We have applied dedicated and tailor-made interventions and intubation techniques.

In chapter 2 we have explored and compared several techniques for delivery of substrates to the colonic region. Depending on the research question and subsequently the amounts of substrate and target region, we have provided evidence for three feasible techniques for use in in vivo studies. Since the accessibility of the human colon has always hampered the in vivo studies in the human colon, these techniques have great potential. Especially the oro-cecal catheter to target and sample locations in the distal ileum or proximal colon and the use of enemas to target the distal colon have proven to be valuable tools for in vivo research and should be further optimised. The oro-cecal catheter has been developed for use in human studies. For the studies described in this thesis, the enemas were chosen as tool to deliver substrates to the distal colon. Enemas have been used because of their ability to deliver relatively large quantities of substrate and even more importantly, they provide a delivery system that subjects can use by themselves for longer periods at home.

The substrate for the research in this thesis was butyrate, one of the short chain fatty acids (SCFA) that is produced as end-product of saccharolytic fermentation in the colon. 
Non-digestible carbohydrates are generally assumed to beneficially modulate colonic health. This benificial effect at least in part results from the ability of non-digestible carbohydrates to increase intraluminal concentrations of short chain fatty acids, such as butyrate. The effectiveness of SCFAs to promote gut and systemic health has not convincingly been established, as most studies conducted so far focussed on modulation of specific health biomarkers, and not on symptom occurrence or disease progression. The studies presented in this thesis contribute to the already present body of evidence that these SCFAs are beneficial for maintaining optimal gut health in healthy individuals and, thus, support current dietary stategies to increase fiber- or prebiotic intake. Importantly, we have shown that patients suffering from the functional bowel disorder Irritable Bowel Syndrome (IBS) clearly benefit from increased intracolonic concentrations of SCFAs. We have demonstrated that these patients perceived significantly less pain, and urge to defecate as measured with the balloon distensions during the barostat procedure after colonic exposure to butyrate compared to placebo.

IBS is a heterogeneous disorder that affects a substantial proportion of our Western society. About $5-15 \%$ of the Dutch population suffer from IBS and associated abdominal complaints like diarrhoea or constipation. This large group of people is confronted with daily sensations of abdominal pain and discomfort, which limits them in their social life and work performance. Patients typically seek for relief of their symptoms, resulting in a high health care consumption. Although only $30 \%$ of IBS patients consults a general practitioner, an estimated amount of over $€ 750$ million is spend on direct costs of health care visits. ${ }^{1}$ This estimation is based on direct costs and does not include absentism from work and reduced productivity. As there is no generally accepted and proven effective treatment for this condition, most patients are actively searching for alternative (dietary) strategies to relieve their abdominal complaints.

Given the large number of IBS patients, the impact on daily life and the impairment in patient reported outcome measures, developing a strategy or therapy targeting to control abdominal symptoms holds great potential for health care professionals but also for nutritional and pharmaceutical industries. One example of an innovative product development in this field has been mentioned in the general discussion of this thesis. Butyrylated starch could be a useful approach to add high amounts of butyrate to food products, without creating problems with osmolarity or consumers being exposed to the typical smell of butyrate. In the case that further research shows that addition of butyrylated starch to existing products would improve colonic health in the general population or even lead to symptom relief and an increase in quality of life for consumers suffering from IBS, this may have big implications for both health care and food industry. 
The potential clinical value of the findings with respect to benifical effects of butyrate on colorectal function in IBS has been evaluated and was initially considered suitable for patent application. Indeed such action has been undertaken and the patent was filed based on the beneficial effects of butyrate to improve abdominal symptoms in healthy volunteers and IBS patients. ${ }^{2}$ This patent application has expired and up to now no further action has been undertaken. We consider the concept to develop novel food products that potentially affect visceral perception and reduce gastrointestinal symptoms to be valid and to hold great potential. Self management is an important item in IBS and patients actively seek for products and life style adjustments in order to reduce symptoms and improve their quality of life.

The rectal barostat has been validated as instrument to quantify visceroperception. Many studies with the barostat have focussed on optimising methodology, stadardising protocols and whenever possible, to reduce burden for patients and researchers or clinicians. The optimisation of the barostat distension protocol for the measurement of rectal (hyper)sensitivity we proposed in chapter 6 may have implications for both the scientific and clinical setting. Once validated, a shorter protocol, which is less prone for errors would significantly decrease patient burden and potentially reduce health care costs. Standardisation, validation, implementation of the generally accepted method of barostat measurement will help clinical- and research groups to compare and exchange data. In chapter 6 we propose to simplify the method and thereby increasing overall acceptance. 


\section{Referenties}

1. Flik CE, Laan W, Smout AJ, de Wit NJ. Irritable bowel syndrome patients diagnosed in primary and secondary care; what explains the difference in health care costs. UEG13-ABS-4598.

2. Vanhoutvin S, Troost FJ, Brummer RJ, Venema K, Hamer HM, Jonkers D. W02009154463 - Butyrate as a medicament to improve visceral perception in humans; Patent application, 2009. 


\section{Dankwoord}




\section{Dankwoord}

Eindelijk, hier ligt ie dan! Nu is het klaar en is dit dankwoord mijn kans om iedereen te bedanken. Zoals velen mij zullen kennen houd ik van kort, en dit is mijn kans.

Terugkijkend op de afgelopen periode kan ik niet anders zeggen dan dat ik in alle opzichten enorm veel geleerd heb. Dit proefschrift was er nooit gekomen zonder de hulp van een heleboel mensen, zowel op het werk als privé.

Uit angst iemand te vergeten wil ik me er niet aan wagen namen te noemen.

Bij deze wil ik dan ook iedereen bedanken die een positieve bijdrage heeft geleverd gedurende mijn promotie en het tot stand komen van dit proefschrift. 
Curriculum Vitae 


\section{Curriculum Vitae}

Steven Vanhoutvin was on born on March 5th 1979 in Weert, the Netherlands. He graduated from his study, Health Sciences at the University of Maastricht, in March 2003. After his graduation he worked as a research assistent for NutriScience, a research and consultancy organization specialized in functional foods. In January 2005 he started his PhD in the TI Food and Nutrition project C-012 "Microbe-mediated Gut Metabolism" were he studied the effects of butyrate (a short chain fatty acid) on different parameters of colonic health with special emphasis on validation of in vivo sampling techniques. From March 2010 Steven started as a clinical research associate for the gastroenterology department at the Netherlands Cancer institute (NKI-AVL). He facilitates the logistics of research projects, from protocol writing and submissions to patient recruitment and datamanagement. In January 2013 he started part-time at a new position as contract manager clinical trials. In this position he runs negotiations of the contracts and budgets of all clinical trials in the institute.

\section{Publications}

1. Vanhoutvin SA, Troost FJ, Jonkers DMAE, Lindsey PJ, Hamer HM, Venema K, GH, Brummer RJM, Masclee AAM. Rectal butyrate instillation decreases visceral perception in IBS patients. Submitted

2. Haanstra JF, Al-Toma A, Dekker E, Vanhoutvin SA, Nagengast FM, Mathus-Vliegen EM, van Leerdam ME, de Vos Tot Nederveen Cappel WH, Sanduleanu S, Veenendaal RA, Cats A, Vasen HF, Kleibeuker $\mathrm{JH}$, Koornstra JJ Prevalence of small-bowel neoplasia in Lynch syndrome assessed by video capsule endoscopy. Gut, 2014.

3. Keszthelyi D, Troost FJ, Jonkers DM, Helyes Z, Hamer HM, Ludidi S, Vanhoutvin S, Venema K, Dekker J, Szolcsányi J, Masclee AA. Alterations in mucosal neuropeptides in patients with irritable bowel syndrome and ulcerative colitis in remission: a role in pain symptom generation? EurJ Pain 2013;17:1299-1306.

4. van Heel NC, Haringsma J, Boot H, Cats A, Vanhoutvin SA, Kuipers EJ. Comparison of 2 expandable stents for malignant esophageal disease: a randomized controlled trial. Gastrointest Endosc 2012;76:52-58.

5. Haanstra JF, de Vos Tot Nederveen Cappel WH, Gopie JP, Vecht J, Vanhoutvin SA, Cats A, van der Zaag-Loonen HJ, Langers AM, Bergmann JH, van de Meeberg PC, Dekker E, Kleibeuker JH, Vasen HF, Nagengast FM, van Duijvendijk P. Quality of life after surgery for colon cancer in patients with Lynch syndrome: partial versus subtotal colectomy. Dis Colon Rectum 2012;55:653-659.

6. Vanhoutvin SA, Troost FJ, Kilkens TO, Lindsey PJ, Jonkers DM, Venema K, Masclee A, Brummer RJ. Alternative procedure to shorten rectal barostat procedure for the assessment of rectal compliance and visceral perception: a feasibility study. J Gastroenterol 2012;47:896-903.

7. Ludidi S, Conchillo JM, Keszthelyi D, Koning CJ, Vanhoutvin SA, Lindsey PJ, Leufkens AM, Kruimel JW, Jonkers DM, Masclee AA. Does meal ingestion enhance sensitivity of visceroperception assessment in irritable bowel syndrome? Neurogastroenterol Motil 2012;24:47-53. 
8. Hamer HM, Jonkers DM, Vanhoutvin SA, Troost FJ, Rijkers G, de Bruïne A, Bast A, Venema K, Brummer RJ. Effect of butyrate enemas on inflammation and antioxidant status in the colonic mucosa of patients with ulcerative colitis in remission. Clin Nutr 2010;29:738-744.

9. Hamer HM, Jonkers DM, Renes IB, Vanhoutvin SA, Kodde A, Troost FJ, Venema K, Brummer RJ. Butyrate enemas do not affect human colonic MUC2 and TFF3 expression. Eur J Gastroenterol Hepatol 2010;22:1134-1140.

10. Vanhoutvin SA, Troost FJ, Hamer HM, Lindsey PJ, Koek GH, Jonkers DM, Kodde A, Venema K, Brummer RJ. Butyrate-induced transcriptional changes in human colonic mucosa. PloS One 2009;4:e6759.

11. Vanhoutvin SA, Troost FJ, Kilkens TO, Lindsey PJ, Hamer HM, Jonkers DM, Venema K, Brummer RJ. The effects of butyrate enemas on visceral perception in healthy volunteers. Neurogastroenterol Motil 2009;21:952-e76.

12. Hamer HM, Jonkers DM, Loof A, Vanhoutvin SA, Troost FJ, Venema K, Kodde A, Koek GH, Schipper RG, van Heerde WL, Brummer RJ. Analyses of human colonic mucus obtained by an in vivo sampling technique. Dig Liver Dis 2009;41:559-564.

13. Hamer HM, Jonkers DM, Bast A, Vanhoutvin SA, Fischer MA, Kodde A, Troost FJ, Venema K, Brummer RJ. Butyrate modulates oxidative stress in the colonic mucosa of healthy humans. Clin Nutr 2009;28:88-93.

14. Hamer HM, Jonkers D, Venema K, Vanhoutvin S, Troost FJ, Brummer RJ. Review article: the role of butyrate on colonic function. Aliment Pharmacol Ther 2008;27:104-119.

15. Kuipers H, Brouwer T, Dubravcic-Simunjak S, Moran J, Mitchel D, Shobe J, Sakai H, Stray-Gundersen J, Vanhoutvin S. Hemoglobin and hematocrit values after saline infusion and tourniquet. Int J Sport Med 2005;26:405-408. 


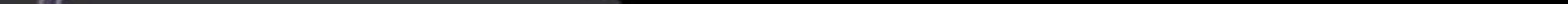

